\title{
Contents
}

\section{Phase coexistence in nuclei}

\section{F. Gulminelli}

Phase transitions in finite systems 3

1. Equilibrium and information 3

2. Generalities about phase transitions 6

3. Mean field approximation and Metropolis sampling 11

4. Finite systems: getting more from pushing less 19

5. A "typical" finite system: the explosion of a supernova 39

6. Abnormal topology of event distributions 45

7. First order phase transitions and abnormal fluctuations 61

2 Applications to nuclear multifragmentation 71

1. Equilibria out of equilibrium 73

2. Heat capacity measurements 83

3. Coulomb effects 95

4. Other signals of phase transitions 102

\section{Outlooks}





\title{
Phase coexistence in nuclei
}

\section{F. Gulminelli ${ }^{1, *}$}

\begin{abstract}
In this work the general theory of first order phase transitions in finite systems is discussed, with a special emphasis to the conceptual problems linked to a thermodynamic description for small, short-lived systems de-exciting in the vacuum as nuclear samples coming from heavy ion collisions. After a short review of the general theory of phase transitions in the framework of information theory, we will present the different possible extensions to the field of finite systems. The concept of negative heat capacity, developed in the early seventies in the context of self-gravitating systems, will be reinterpreted in the general framework of convexity anomalies of thermostatistical potentials. The connection with the distribution of the order parameter will lead us to a definition of first order phase transitions in finite systems based on topology anomalies of the event distribution in the space of observations. A careful study of the thermodynamic limit will provide a bridge with the standard theory of phase transitions and show that in a wide class of physical situations the different statistical ensembles are irreducibly inequivalent. In the second part of the paper we will apply the theoretical ideas developed in the first part to the possible observation of a liquid-to-gas-like phase transition in heavy ion collisions. The applicability of equilibrium concepts in a dynamical collisional process without boundary conditions will first be critically discussed. The observation of abnormally large partial energy fluctuations in carefully selected samples of collisions detected with the MULTICS-Miniball and INDRA array will then be reported as a strong evidence of a first order phase transition with negative heat capacity in the nuclear equation of state.
\end{abstract}

1. LPC Caen (IN2P3-CNRS/Ensicaen et Université), 14050 Caen Cedex, France.

* Member of the Institut universitaire de France

Ann. Phys. Fr. 29 • No $6 \bullet 2004$

(C) EDP Sciences 


\section{Résumé}

\section{Coexistence de phase dans les noyaux}

Ce papier présente une revue de la théorie générale des transitions de phase du premier ordre dans les petits systèmes, avec une attention particulière aux problèmes conceptuels liés à une description thermodynamique des systèmes ouverts, à petit nombre de degrés de liberté et courte durée de vie tels les systèmes nucléaires issus d'une collision d'ions lourds. Après une revue rapide de la théorie générale des transitions de phase dans le cadre de la théorie de l'information, nous discutons les différentes extensions au domaine des systèmes finis. Le concept d'une capacité calorifique négative, introduit dans les années 70 dans le contexte des systèmes autogravitants, est re-interprété dans le cadre plus général des anomalies de courbure de potentiels thermodynamiques généralisés. La connexion avec la distribution du paramètre d'ordre conduit alors à une définition des transitions de phase du premier ordre dans les systèmes finis, basée sur les anomalies topologiques de la distribution des événements dans l'espace des observations. Une étude détaillée de la limite thermodynamique fournit un pont avec la théorie standard des transitions de phase, et montre que dans une large classe de situations physiques les ensembles statistiques sont irréductiblement non équivalents. Dans la deuxième partie de cet ouvrage nous appliquons les idées théoriques développés dans la première partie à la possible observation d'une transition de phase du type liquide gaz dans les collisions d'ions lourds. La possibilité d'appliquer des concepts d'équilibre dans un processus dynamique collisionnel avec continuum non lié est discuté de façon critique. L'observation de fluctuations d'énergie partielle anormalement grandes dans des échantillons sélectionnées de collisions étudiées à l'aide de détecteurs MULTICS-Miniball et INDRA est reportée et interprétée comme une évidence expérimentale forte d'une transition de phase du premier ordre avec capacité calorifique négative dans l'équation d'état des noyaux. 


\section{Phase transitions in finite systems}

\section{Equilibrium and information}

\subsection{States and observables $[1,2]$}

Modern physics associates to every physical system two different types of objects: observables that characterize the measurable physical quantities and states whose knowledge allows to predict the result of experiments. From the microscopic point of view, single realizations of systems with $N$ degrees of freedom are characterized by a pure state (or microstate), that is a wave function $\left|\Psi_{N}\right\rangle$ in quantum mechanics or a point in the $2 N$-dimensional phase space $\mathbf{s}=\left(q_{1}, q_{2}, \ldots, q_{N} ; p_{1}, p_{2}, \ldots, p_{N}\right)$, with $q_{i}$ and $p_{i}$ the position and momentum of each degree of freedom, in classical mechanics. If systems are sufficiently complex, the exact state is in general impossible to define and each actual realization corresponds to a microstate $(n)$ with the probability $p^{(n)}$. In such a realistic case, one rather speaks of mixed states (or macrostates) described by the density

$$
\widehat{D}=\sum_{n}\left|\psi^{(n)}\right\rangle p^{(n)}\left\langle\psi^{(n)}\right| \text { or } \widehat{D}(\mathbf{s})=\sum_{n} \delta\left(\mathbf{s}-\mathbf{s}^{(n)}\right) .
$$

Observables are operators defined on the Hilbert space or classically real functions of $2 N$ real variables. The information that can be associated to the system is the ensemble of expectation values of the observables $\widehat{A_{l}}$ on the state, i.e. the ensemble of observations $\left\langle\widehat{A}_{l}\right\rangle=\sum_{n} p^{(n)} A_{l}^{(n)}$ where $A_{l}^{(n)}$ is the actual result of a measurement on the realization $(n)$. In the quantum case $\left\langle\widehat{A_{l}}\right\rangle=\sum_{n} p^{(n)}\left\langle\psi^{(n)}\left|\widehat{A_{l}}\right| \psi^{(n)}\right\rangle=\operatorname{Tr}\left(\widehat{D} \widehat{A_{l}}\right)$. Both for pure and mixed states, if the information on the system is complete at the initial time, this stays true at any time because the dynamical evolution of states is governed by the deterministic Liouville Von Neumann equation $\partial_{t} \widehat{D}=\{\widehat{H}, \widehat{D}\}$ where $\widehat{H}$ is the Hamiltonian of the system and where $\{.,$.$\} is the commutator$ divided by $i \hbar$ in quantum mechanics which reduces to the usual Poisson bracket at the classical limit. However in the case of complex systems, the initial conditions are in general incompletely known and an exact solution of the Liouville Von Neumann equation is out of reach. In general only a small set of pertinent observables is known at any time which is sufficient to determine the state (i.e. the totality of the $p^{(n)}$ ) because of the complexity of the density operator. 


\subsection{The fundamental postulate of statistical mechanics $[1,2]$}

Let us consider an experiment that can lead to $N_{\text {tot }}$ different outcomes, each of them associated to a (a priori unknown) probability of occurrence $p^{(i)}, i=1, \ldots, N_{\text {tot }}$. If the $N_{\text {tot }}$ possible outcomes are grouped into $m$ families of $n$ results each, $N_{\text {tot }}=$ $m \times n$, the prediction of the outcome of the experiment can be divided into two successive steps: first find out to which family out of the $m$ possible ones the result belongs, then determine the actual outcome out of the $n$ elements of the chosen family.

The incompleteness of the available information can be measured through the lack of information or statistical entropy $S$.

Let us enumerate some fundamental properties of $S$ :

- the lack of information must grow with the number of possible results $S\left(N_{1}\right)>S\left(N_{2}\right) \forall N_{1}>N_{2}$;

- the information cannot depend on the number of steps through which it is collected $S\left(N_{t o t}\right)=S(m \times n)=S(m)+S(n)$.

It is easy to show that the ensemble of these properties can only be fulfilled within a constant by the function

$$
S=-\sum_{n} p^{(n)} \ln p^{(n)}=-\operatorname{Tr}(\widehat{D} \ln \widehat{D})
$$

It may be interesting to know that if the additivity property of the information is relaxed, it is possible to construct a non-extensive extension of the Shannon theory based on the so-called $q$-statistics which has interesting applications in out of equilibrium situations as in the case of turbulent flows [3]. In the following we will limit ourselves to the standard Shannon entropy introduced above (but we will briefly come back to the problem of the information kernel in Chap. 3).

Once the missing information is defined through equation (1), the fundamental postulate of statistical mechanics can be expressed as follows: "The statistical distribution of microstates usually called the equilibrium is the one which maximizes the statistical entropy within the external constraints (i.e. the pertinent information) imposed to the system".

Indeed any other distribution would introduce an extra piece of information, in contrast with the statement that all the available information is given by the constraint.

It is important to remark that this postulate, though certainly intuitive and elegant, does not necessarily imply that the theory has any predictive power: the fact that we have only a limited amount of information on a system does not necessarily mean that the information contained in the system is objectively limited. In the following we shall anyway keep the fundamental postulate as the only reasonable working hypothesis in a complex system, and we will come back to its possible justifications from the dynamics in the second part of this work. 
The fundamental postulate of statistical mechanics allows to determine the equilibrium values of the state probabilities $p^{(n)}$. This task is easily accomplished with the method of Lagrange multipliers.

\subsection{Equilibrium and statistical ensembles [4]}

Let us use the method of Lagrange multipliers to maximize the statistical entropy $S=-\operatorname{Tr} \hat{D} \ln \hat{D}$ under the constraint of a given set of $L$ observations $\left\langle\hat{A}_{l}\right\rangle$.

This situation corresponds to the $L$ constraints $\operatorname{Tr} \hat{D} \hat{A}_{l}=\left\langle\hat{A}_{l}\right\rangle$ plus the extra constraint of the normalization of probability $\operatorname{Tr} \hat{D}=1$ which can be incorporated as an additional observable $\hat{A}_{0}=1$. We can define an auxiliary function $Y$ as

$$
Y=-\operatorname{Tr} \hat{D} \ln \hat{D}-\sum_{l=0}^{L} \lambda_{l} \operatorname{Tr} \hat{D} \hat{A}_{l} .
$$

The extremum corresponds to $\delta Y=0$, with no restrictions on the variation $\delta \hat{D}$ of the density matrix $\hat{D}$, leading to the condition $\ln \hat{D}+1+\sum_{l=0}^{L} \lambda_{l} \hat{A}_{l}=0$. The solution is the density matrix at equilibrium, which is a function of the Lagrange multipliers $\lambda_{l}$,

$$
\hat{D}^{0}=\frac{1}{Z} \exp \left(-\sum_{l=1}^{L} \lambda_{l} \hat{A}_{l}\right)
$$

where we have already taken care of the normalization constraint by introducing the partition sum

$$
Z=\operatorname{Tr} \exp \left(-\sum_{l=1}^{L} \lambda_{l} \hat{A}_{l}\right)
$$

The link between a constraint $\left\langle\hat{A}_{l}\right\rangle$ (or observation, or extensive variable) and the associated Lagrange multiplier $\lambda_{l}$ (or thermodynamically conjugated intensive variable) is given by an equation of state,

$$
\left\langle\hat{A}_{l}\right\rangle=-\frac{\partial \ln Z}{\partial \lambda_{l}}
$$

It is also possible to express $\lambda_{l}$ as a function of $\langle A\rangle$ by inverting the equation of state. Indeed the equilibrium corresponding to the considered constraints is associated to a value for the statistical entropy

$$
S=\operatorname{Tr} \hat{D}^{0} \ln \hat{D}^{0}=\sum_{l} \lambda_{l}\left\langle\hat{A}_{l}\right\rangle+\ln Z
$$

This last equation, known as a Legendre transform, gives the relation between the entropy and the partition sum and implies for the Lagrange multipliers

$$
\lambda_{l}=\frac{\partial S}{\partial\left\langle\hat{A}_{l}\right\rangle}
$$


It should be noticed that while $D^{0}$ and $Z$ are functions of the intensive variables $\left(\lambda_{l}\right)$, the Legendre transform $S$ is a function of the associated extensive variables $\left\langle\hat{A}_{l}\right\rangle$.

Using equations (2-4) the whole thermodynamics of the system can be calculated if the constraints $\left\langle\hat{A}_{l}\right\rangle$ are known. It is important to remark that this formalism is completely general in the sense that it can be applied for an arbitrary number of bodies with no need of a thermodynamic limit, and that all observables (and not only variables conserved by the dynamics) can play the role of constraints. Moreover the maximization of entropy as a tool to deal with the general problem of missing information can be extended in dynamical situations and has shown to be a fruitful approach in the field of stochastic quantum transport [5].

The usual ensembles of standard thermodynamics can also be obtained as applications of this general theory. Let us consider for example the case where the only constraint is the energy $\langle E\rangle=\operatorname{Tr}\left(\hat{D}^{0} \hat{H}\right)=\sum_{n} p_{n} E^{(n)}$ associated with the Lagrange multiplier $\beta$. The probability of the $n$th energy eigenstate is then $p_{n}^{0}=\left(1 / Z_{\beta}\right) \exp \left(-\beta E^{(n)}\right)$ while the energy probability distribution reads $p_{\beta}(E)=$ $\left(W(E) / Z_{\beta}\right) \exp (-\beta E)$ where $W(E)$ is the number of states corresponding to an energy $E$. The Lagrange multiplier $\beta$ has the physical meaning of the inverse of the temperature $T=\beta^{-1}$. The relation between the average energy and the temperature is given by the equation of state $\langle E\rangle=-\partial_{\beta} \ln Z_{\beta}$ and the Legendre transform $S(\langle E\rangle)=\ln Z_{\beta}+\beta\langle E\rangle$ represents the well known relation between the canonical entropy and the free energy $F_{T}=-\beta^{-1} \ln Z_{\beta}$.

The microcanonical ensemble can also be obtained from this general theory considering that in the absence of any constraint (except the normalization of probabilities) all states must be equiprobable. The microcanonical entropy is then obtained as the expression of the Shannon entropy within the equilibrium distribution $p_{i}^{0}=1 / W(E), S(E)=-\sum_{i=1}^{W} W^{-1} \ln W^{-1}=\ln W$.

\section{Generalities about phase transitions}

Generally speaking, for a given value of the control parameters (or intensive variables) $\lambda_{l}$, the properties of a substance are univocally defined, i.e. the conjugated extensive variables $\left\langle\hat{A}_{l}\right\rangle$ have a unique value unambiguously defined by the corresponding equation of state. For instance the volume occupied by $n$ moles of an ideal gas at a given pressure $P$ and temperature $T$ is given by $V=n R T / P$. In reality we have seen in the previous chapter that extensive variables, being by definition expectation values of operators, are associated with a probability distribution, unless the system is described by a pure state. The intuitive expectation that extensive variables at equilibrium have a unique value therefore means that the probability distribution is narrow and normal, such that a good approximation can be obtained by replacing the distribution with its most probable value.

In this case, as we will see in Section 2.1 (Chap. 1), the Legendre transform gives an exact mapping between the standard intensive ensembles in which the control parameter is intensive (or equivalently only the average of the extensive variable 
is known), and the more exotic extensive ensembles where an extensive variable is controlled event by event, demonstrating the equivalence between the different statistical ensembles. In the following we will often take as a paradigm of intensive ensembles the canonical ensemble for which the inverse of the temperature $\beta^{-1}$ (or equivalently the average energy $\langle E\rangle$ ) is controlled, while the archetype of the extensive ensemble will be the microcanonical one for which the energy is strictly conserved on an event by event basis.

The normality of probability distributions is usually assumed based on the central limit theorem that we will briefly review in Section 2.2 (Chap. 1). However some situations exist in which the probability distributions of extensive variables are abnormal, and for example bimodal: in this case two different properties (phases) coexist for the same value of the intensive control variable. A first elementary description of phase coexistence using this intuitive bimodality argument will be given at the end of Section 2.2 (Chap. 1).

The topological anomalies of probability distributions and the failure of the central limit theorem in phase coexistence imply that in a first order phase transition the different statistical ensembles are in general not equivalent and different phenomena can be observed depending on the fact that the controlled variable is extensive or intensive. This general statement will be developed in great detail in Section 4 (Chap. 1) and its far reaching consequences will be analyzed in Section 6 (Chap. 1).

\subsection{The difference between Laplace and Legendre}

We have seen in the last section that the relation between the different thermostatistical potentials is given by the Legendre transform. It is important to distinguish between transformations within the same statistical ensemble as the Legendre transform (which gives for instance the link between the canonical partition sum and the canonical entropy) and transformations between different ensembles which are instead given by non linear integral transforms. Let us consider energy as the extensive observable and temperature as the conjugated intensive one. The definition of the canonical partition sum is

$$
Z_{\beta}=\sum_{n} \exp \left(-\beta E^{(n)}\right)
$$

where the sum runs over the available eigenstates $n$ of the Hamiltonian. If energy can be treated as a continuous variable this equation can be written as

$$
Z_{\beta}=\int_{0}^{\infty} d E W(E) \exp (-\beta E),
$$

which is nothing but a Laplace transform between the canonical partition sum and the microcanonical entropy $S_{E}=\ln W(E)$. If the integrand $f(E)=W(E) \exp (-\beta E)$ is a strongly peaked function, the integral can be replaced by the maximum $f(\bar{E})$

$$
Z_{\beta} \approx W(\bar{E}) \exp (-\beta \bar{E})
$$


which can be rewritten as

$$
\begin{array}{r}
\ln Z_{\beta} \approx S_{\bar{E}}-\beta \bar{E}, \\
\text { or introducing the free energy } F_{T}=-\beta^{-1} \ln Z_{\beta} \\
F_{T} \approx \bar{E}-T S_{\bar{E}} .
\end{array}
$$

Equation (9) has the structure of an approximate Legendre transform, and shows that in the saddle point approximation equation (8) the ensembles differing at the level of constraints acting on a specific observable (here energy) differ only by a simple linear transformation. We will see in the next section and in more details in Section 6 (Chap. 1) that however the saddle point approximation equation (8) can be highly incorrect close to a phase transition. In particular, when the canonical distribution of energy is bimodal, a unique saddle point approximation becomes inadequate. In this case equation (9) cannot be applied, and equation (7) is the only possible transformation between the different ensembles.

To summarize one should not confuse

- the link between the thermodynamic potential of the intensive and of the extensive ensemble which is given by a Laplace transform. This Laplace transform may lead to an approximate Legendre transformation only if the distribution is normal;

- the exact Legendre transform between the entropy of the intensive ensemble and the corresponding thermodynamic potential.

This simply corresponds to the fact that the microcanonical and canonical entropies can be very different.

\subsection{The central limit theorem and phase coexistence}

The typical representation of the probability distribution of any generic random variable depending on a not too small number of degrees of freedom is a Gaussian distribution. The very general validity of the Gaussian is due to one of the most important theorems of statistics, the Laplace central limit theorem. Let us consider an extensive observable $E$ (i.e., energy) that can be written as the sum of $I$ independent contributions (i.e. the energy of the different particles constituting the system) $E=\sum_{i}^{I} e_{i}$, where the $e_{i}$ follow an arbitrary probability distribution with the unique requirement that the global variance $\sigma_{E}^{2}=\sum_{i}^{I}\left(\left\langle e_{i}^{2}\right\rangle-\left\langle e_{i}\right\rangle^{2}\right) / I$ is finite. Then the central limit theorem states that the distribution of $E$ tends to a Gaussian distribution with a width linearly decreasing with the number of degrees of freedom

$$
\lim _{I \rightarrow \infty} p(E)=\frac{1}{\sqrt{2 \pi \sigma^{2} / I}} \exp \left(-\frac{(E-\langle E\rangle)^{2}}{2 \sigma^{2} / I}\right) .
$$

According to the central limit theorem, at the thermodynamic limit the distribution of an extensive variable $p(E)$ tends to a $\delta$-function, implying, as we have 
mentioned at the beginning of the section, that the properties of the system are univocally defined by the value of the intensive parameter that controls the asymptotic value of $\langle E\rangle$ through the appropriate equation of state.

In most cases a few tens of particles are enough for the Gaussian approximation to be correct, meaning that the limit appearing in equation (10) can be neglected in practical applications. Another consequence of the central limit theorem is that the Laplace transform becomes equivalent to a Legendre transform as we have discussed in the preceding section, leading to the equivalence of statistical ensembles.

However a situation can occur, in which the probability distribution is bimodal and never tends to a Gaussian. Such a situation is called a first order phase transition. This patent violation of the central limit theorem is due to the fact that phase transitions are associated to long range correlations and the independence hypothesis between the different degrees of freedom breaks down.

Let us illustrate the standard picture of phase coexistence within a simple example. Consider a molecular system in the canonical ensemble characterized by the free energy $F=-T \ln Z=\langle E\rangle-T S$. As we have demonstrated in Section 1.3 (Chap. 1) the maximization of the statistical entropy with the energy constraint is equivalent to the minimization of the free energy. At low temperature a minimization of $F$ is approximately equivalent to a minimization of $\langle E\rangle$ : the equilibrium state of the system will be given by a compact configuration (a crystal or a liquid) with free energy $F_{L}(A, V)$. On the other side at high temperature the minimization of $F$ corresponds to a maximization of the canonical entropy, which will be achieved by a disordered rarefied state (a gas phase) with free energy $F_{G}(A, V)$. Phase coexistence means that at an intermediate temperature the two free energy solutions are allowed, giving for the total free energy

$$
F(A, V)=F_{L}\left(A_{L}, V_{L}\right)+F_{G}\left(A_{G}, V_{G}\right),
$$

where $A_{L}, V_{L}\left(A_{G}, V_{G}\right)$ are the fractions of total number of molecules $A$ and volume $V$ belonging to the ordered (disordered) phase

$$
A=A_{L}+A_{G} ; \quad V=V_{L}+V_{G} .
$$

The equilibrium sharing of $A$ and $V$ is given by the minimization of the free energy

$$
\frac{\partial F}{\partial A_{L}}=\frac{\partial F_{L}}{\partial A_{L}}-\frac{\partial F_{G}}{\partial A_{G}}=0 ; \quad \frac{\partial F}{\partial V_{L}}=\frac{\partial F_{L}}{\partial V_{L}}-\frac{\partial F_{G}}{\partial V_{G}}=0,
$$

implying the equality for the intensive variables conjugated to the mass number and the volume, namely the chemical potential and the pressure

$$
p_{L}=p_{G} ; \quad \mu_{L}=\mu_{G} .
$$

This procedure can be generalized to any statistical ensemble. If we consider for example the microcanonical ensemble, the absence of constraints means that the thermostatistical potential is directly the microcanonical entropy

$$
S(A, E, V)=\ln W(A, E, V)=S_{L}\left(A_{L}, E_{L}, V_{L}\right)+S_{G}\left(A_{G}, E_{G}, V_{G}\right),
$$


with the extra conservation law $E=E_{L}+E_{G}$. The extremization of $S$ respect to $V$ and $A$ gives again the equality of the chemical potential and pressure for the two coexisting phases, while the derivative respect to the energy gives

$$
T_{L}=T_{G},
$$

where we have defined the microcanonical temperature as $T^{-1}=\partial_{E} S$ in analogy with the canonical Legendre transform $\beta=\partial_{\langle E\rangle} S_{\beta}$ (the justification of the physical meaning of $\partial_{E} S$ as an inverse temperature is postponed to Sect. 4, Chap. 1). Equilibrium between the two phases is characterized by the equality of the temperatures.

On the other hand, the conjugated extensive variables are different in the two phases $E_{L}<E_{G}$. This means that at the transition temperature $T_{t r}=T_{L}=T_{G}$ the energy is discontinuous at the phase transition (latent heat).

To summarize, in this standard view first order phase transitions are characterized by

- the presence of two phases in contact;

- a discontinuity in (one ore more) first order derivatives of the thermostatistical potential (energy, volume, mass number...).

To obtain this result we have written the thermostatistical potential as a simple sum of the contributions of the two phases (Eqs. $(11,12))$. This is true only if the free energy (or entropy) of the interface between the two phases is negligible, i.e. for large systems interacting through short range forces.

In the next sections we will illustrate this standard view of first order phase transitions within the Ising model.

The additivity hypothesis of the thermostatistical potential breaks down for finite systems, and even in the thermodynamic limit if the forces are long ranged. The far reaching consequences of dropping this approximation will be developed in Section 4 (Chap. 1).

\subsection{The Ising model and its relation to lattice gas}

Let us consider an ensemble of $N$ classical spins which can take one of the two values $s_{k}= \pm 1$ on a lattice under the influence of an external magnetic field $h$ and a constant coupling $J$ between neighbouring sites according to the Hamiltonian

$$
H_{I S}=-h \sum_{k=1}^{N} s_{k}-\frac{J}{2} \sum_{k \neq j}^{N} s_{k} s_{j},
$$

where the second sum extends over closest neighbours.

The Ising model (Eq. (13)) has been originally introduced to give a simple description of ferromagnetism. In reality the phenomenon of ferromagnetism is far too complicated to be treated in a satisfactory way by this oversimplified 
Hamiltonian; however the fact that the Ising model is exactly solvable in 1D and $2 \mathrm{D}$ and that very accurate numerical solutions exist for the three dimensional case makes this model a paradigm of first and second order phase transitions. The other appealing side of the Ising model is its versatility: introduced to explain magnetic phase transitions, it is also well adapted to describe fluid phase transitions. Indeed we can show that a close link exists between the Ising Hamiltonian (Eq. (13)) and the lattice gas Hamiltonian, which is the simplest modelization of the liquid-gas phase transition

$$
H_{L G}=\frac{1}{2 m} \sum_{k=1}^{N} p_{k}^{2} n_{k}-\frac{\varepsilon}{2} \sum_{k \neq j}^{N} n_{k} n_{j}
$$

In the lattice gas model, the same $N$ lattice sites in $D$ dimensions are characterized by an occupation $n_{k}=0,1$ and by a $D$ components momentum vector $\vec{p}_{k}$. Occupied sites (particles) interact with a constant closest neighbour coupling $\varepsilon$.

Because of the transformation $n_{k}=\left(s_{k}+1\right) / 2$, the Ising Hamiltonian $H_{I S}$ can be mapped into the interaction part $H_{L G}^{i n t}$ of the lattice gas Hamiltonian $H_{L G}$. Indeed let us consider the interaction part of the Lattice Gas partition sum in the grancanonical ensemble

$$
Z_{L G}^{\text {int }}=\sum_{n_{1}=0,1} \ldots \sum_{n_{N}=0,1} \exp \left(-\beta\left(H_{L G}^{\text {int }}-\mu A\right)\right),
$$

where $A=\sum_{k}^{N} n_{k}$ is the total number of particles and $\beta, \mu$ are Lagrange multipliers. With the identification $J=\varepsilon / 2$ and $h=(z \varepsilon+\mu) / 2$, it is easy to show that the grancanonical partition sum of the lattice gas interaction Hamiltonian is isomorphous to the canonical partition sum of the Ising model in an external field

$$
\beta\left(H_{L G}^{i n t}-\mu A\right)=\beta H_{I S}+K
$$

where $K$ is a constant. This result implies that all results obtained within the Ising model concerning magnetic transitions can be translated in terms of fluid transitions and vice-versa. In particular the magnetization $m=\left\langle\sum_{k} s_{k}\right\rangle / N$ is related to the matter density $\rho=\left\langle\sum_{k} n_{k}\right\rangle / N$ by $m=2 \rho-1$.

\section{Mean field approximation and Metropolis sampling}

Even for simplified models such as Ising no analytical solution exists for a number of dimensions $D>2$. This is the reason why mean field solutions have been developed. The idea of the mean field approximation is to replace the intractable $\mathrm{N}$-body problem with an approximately equivalent analytical one body problem. 
Let us illustrate this method on the Ising case. If the Hamiltonian is composed of one body terms solely

$$
H_{1 b}=-\sum_{k=1}^{N} h_{k} s_{k}
$$

with $h_{k}$ a generic one body operator, the thermodynamics of the system is solved in one line. Indeed the partition sum in the canonical ensemble reads

$$
Z_{1 b}=\sum_{s_{1}= \pm 1} \ldots \sum_{s_{N}= \pm 1} \exp \left(-\beta H_{1 b}\right)=\prod_{k=1}^{N} z_{k}=(\exp (-\beta h)+\exp (\beta h))^{N},
$$

where the last equality holds if $h_{k}=h \forall k$, and is promptly generalized to the non-local case.

To reduce the Hamiltonian to a one body interaction, the correlations among the different sites have to be neglected, such that the interaction on a given site depends only of the coordinates of the site. The first part of this section is devoted to one of the possible applications of this approximation to the Ising model (Sect. 3.1, Chap. 1) and its consequences for the problem of first order phase transitions in fluids (Sect. 3.2, Chap. 1). We will see that an equivalent one body problem can be formulated, and the two body character of the force results in a self-consistency problem for the equations of state which have to be solved iteratively.

It is important to stress that all mean field approaches are approximations which, because of the intrinsic lack of correlations, are especially bad in phase coexistence. In the recent years, the enormous progress of computing machines has allowed the numerical solution of three dimensional models without any approximation with Monte-Carlo based methods (Sect. 3.3, Chap. 1). These exact solutions clearly show the inherent limitations of mean field approaches, as it will be discussed in Section 4 (Chap. 1).

\subsection{Mean field approximation for the Ising model}

The interaction acting on the $k$-th site in the Ising model (Eq. (13)) is $h_{k}=h+$ $J \sum_{j} s_{j}$, where the sum extends over the first neighbours of site $k$. The simplest way to obtain a one body term is to assume the spin of the $q$ neighbouring sites $s_{j}$ constant all over the lattice and equal to the average magnetization $s_{j} \approx$ $\langle s\rangle=m$. With this simplest version of the mean field approximation the exact interaction is approximated by the interaction the site would experience if the spin distribution was uniform. The Ising Hamiltonian can then be written as a one body Hamiltonian

$$
H_{M F}=-\sum_{k=1}^{N} h_{k} s_{k}+K=-\sum_{k=1}^{N}(h+J q m) s_{k}+K,
$$


within a constant $K$ which has to be determined by imposing that the expectation value of $H_{M F}$ is equal to the mean field energy

$$
E_{M F}=-h N m+E_{M F}^{i n t}=-N\left(h m+\frac{J q}{2} m^{2}\right)
$$

where the last equality is obtained by writing the interaction energy as

$$
E^{i n t}=-\frac{J}{2} \sum_{k=1}^{N} \sum_{j \neq k}\left\langle s_{k} s_{j}\right\rangle \approx-\frac{J}{2} \sum_{k=1}^{N} \sum_{j \neq k}\left\langle s_{k}\right\rangle\left\langle s_{j}\right\rangle=-\frac{J}{2} N q m^{2}
$$

which shows once again that the effect of the mean field approximation is the neglect of two body correlations. The comparison of equation (18) with the expectation value of equation (17) leads to the definition of the constant $K$ as $K=J N q m^{2} / 2$. In fact this energy correction exactly compensates the double counting of the two-body interaction due to the introduction of the average interaction of each spin with all its neighbours. The mean field partition sum, as for equation (16), is factorized in the product of the individual partition sums of the different sites

$$
Z_{M F}=\sum_{s_{1}= \pm 1} \cdots \sum_{s_{N}= \pm 1} \exp \left(-\beta\left(H_{M F}\right)\right)=z^{N}
$$

where

$$
z=\sum_{s= \pm 1} \exp \left(-\beta\left(-(h+J q m) s+\frac{J q}{2} m^{2}\right)\right)=2 \exp \left(-\beta \frac{J q}{2} m^{2}\right) \operatorname{ch}(\beta(h+J q m)),
$$

which leads to a self-consistent equation for the magnetization

$$
m=\tanh (\beta(h+J q m)) .
$$

Equation (20) is represented in Figure 1 in the subcritical, critical and supercritical regime. If the behaviour of the equation of state for $T \geq T_{c}=J q$ is qualitatively similar to the celebrated exact Onsager solution of the two-dimensional Ising model [6], in the first order phase transition regime the mean field solution shows a backbending behaviour with a negative susceptibility $\chi^{-1}=\partial_{h} m$ region. To understand the physical meaning of the backbending, the free energy $F=-\beta^{-1} \ln Z_{M F}$ is shown as a function of magnetization in Figure 2 in the $h=0$ and $h>0$ case. From this figure one can see that the backbending corresponds to a maximum of the free energy, i.e. an instability. Indeed the coexistence between the two phases at different magnetization cannot be obtained in a mean field calculation, because of the intrinsic homogeneity hypothesis $m=\langle s\rangle=$ const. The backbending therefore reflects the instability of the homogeneous mean field solution with zero magnetization, respect to the separation into two distinct phases at $m= \pm m_{0}$. At non zero field the magnetization oriented in the direction of the 


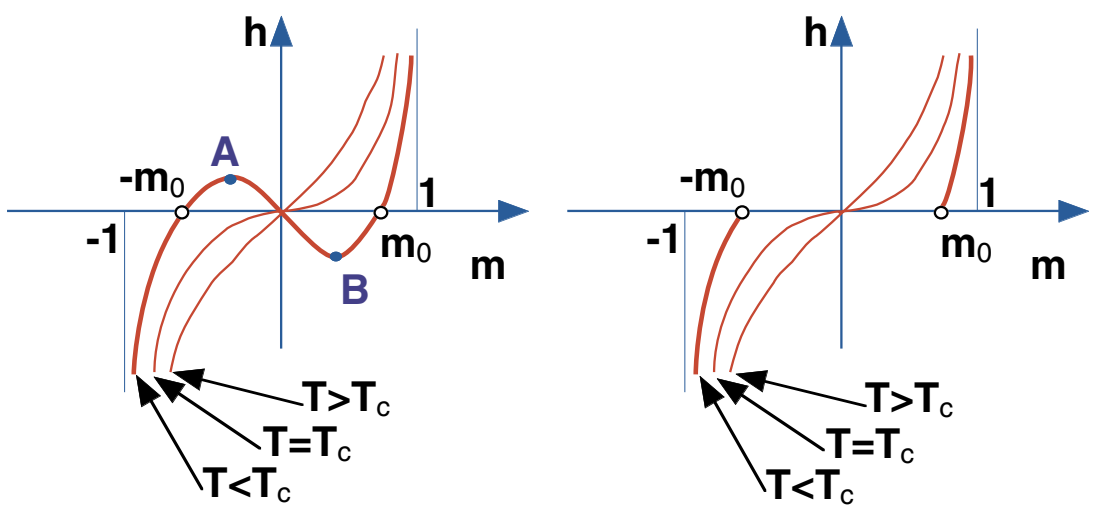

Figure 1. Left side: relation between the average magnetization and the magnetic field at subcritical, critical and supercritical temperature for the three dimensional Ising model in the mean field approximation. Right side: Maxwell construction modifying the subcritical magnetization curve.
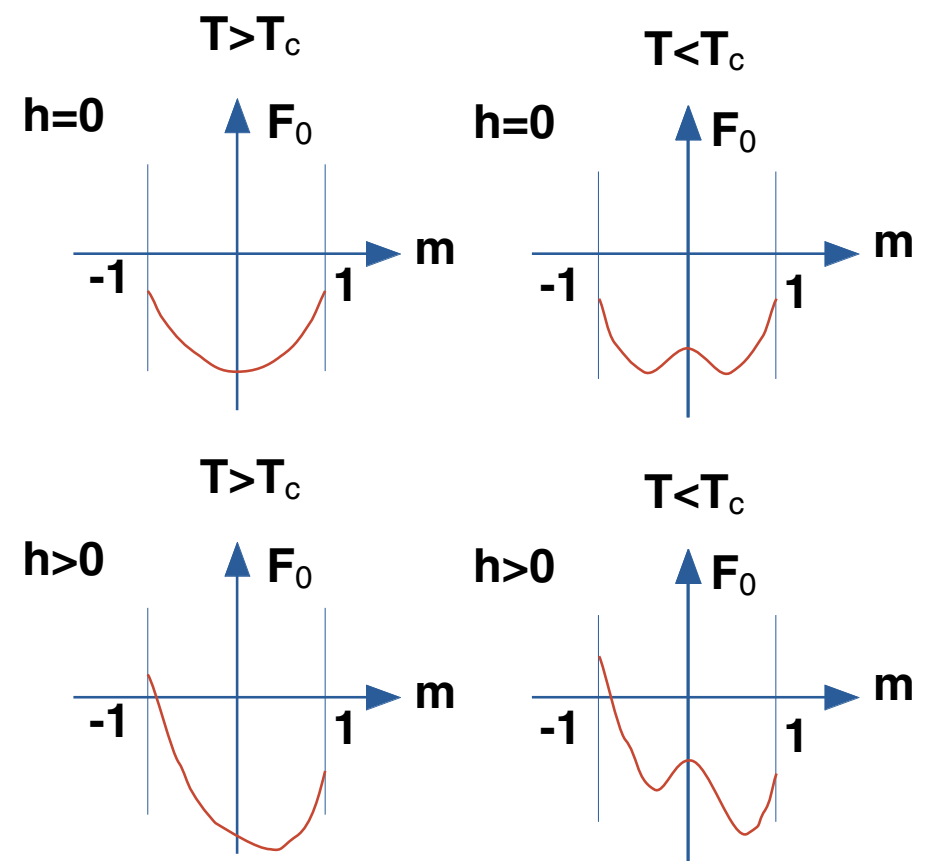

Figure 2. Mean field free energy as a function of magnetization at zero (upper part) and positive (lower part) magnetic field, for a supercritical (left) and a subcritical (right) temperature. 
field has the minimum free energy, therefore it will correspond to the unique equilibrium solution. In the zero field case the two solutions have the same energy. This implies that every linear combination of these solutions

$$
m\left(h=0, T<T_{c}\right)=\alpha m_{0}+(1-\alpha)\left(-m_{0}\right) ; \quad 0<\alpha<1
$$

will have the same free energy; such a linear combination represents the coexistence between the two solutions as we have discussed in Section 2 (Chap. 1), and corresponds to an horizontal straight line in the $F-m$ and in the $h-m$ plane (tangent construction), as shown in the right part of Figure 1.

If the lack of correlations of the mean field is cured by allowing a mixed phase according to equation (21), the usual shape of the phase transition is recovered (discontinuity in the first derivative of the thermodynamic potential).

To conclude this section we would like to comment the difference between a self consistent approach as the mean field approximation and a genuine one body Hamiltonian as in equations $(15,16)$. We have shown in Section 1 (Chap. 1) that the thermodynamics of a system is completely determined once the partition sum is known, since all thermodynamic quantities can be calculated as successive derivatives of $\ln Z$. The Hamiltonian entering the mean field approximation of the partition sum equation (19) differs from the mean field approximation of the Ising Hamiltonian, because of the constant $K$ which we have been forced to add for the Hamiltonian to have the correct expectation value. The constant $K$ in the partition sum represents more than a trivial shift in the energy scale, since $K$ depends on $m$ which in turn is calculated from $\ln Z$ showing the self-consistent character of the approach. Following equations $(15,16)$ one could be tempted to define from the mean field approximation to the Ising Hamiltonian a one body partition sum as

$$
Z_{1 b}=\left(\sum_{s= \pm 1} \exp (-\beta(h+J q m) s)\right)^{N}
$$

and the question arises whether thermostatistical observables can be obtained from the successive derivatives of $Z_{1 b}$.

To answer to this question one has to use the formalism of Section 1 (Chap. 1) and explicitly calculate the statistical entropy

$$
S_{M F}=-\sum_{i} p_{i} \ln p_{i}=-\sum_{i}\left(p_{i}\left(-\beta H_{1 b}^{(i)}-\ln Z_{1 b}\right)\right)=\beta\left\langle H_{1 b}\right\rangle+\ln Z_{1 b},
$$

since the probability distribution for the mean field problem reads $p_{i}=$ $\exp \left(-\beta H_{1 b}^{(i)}\right) / Z_{1 b}$. The expectation of $H_{1 b}$ is readily calculated as

$$
\left\langle H_{1 b}\right\rangle=-N\left(h m+J q m^{2}\right)=-h N m+2 E_{M F}^{i n t} .
$$

The general relation between entropy and free energy $-\beta F \equiv \ln Z_{M F}=S_{M F}-\beta E_{M F}$ finally leads to

$$
\ln Z_{M F}=\ln Z_{1 b}+\beta E_{M F}^{i n t} .
$$


Equation (23) shows that, because of the two body interaction, the partition sum is different from the one body partition sum even in the mean field approximation. In fact the difference comes from the double counting of the two-body interaction if the energy is calculated as $\left\langle H_{1 b}\right\rangle$.

The best way to understand the mean-field approach is to consider mean-field solutions as a trial state to maximize the entropy completed by the constraints $(S-\beta\langle E\rangle)$, i.e. to variationally estimate the free energy $F=-\beta^{-1}(S-\beta\langle E\rangle)$. Then only the mean-field free energy can be considered as a good approximation of the exact free energy leading to $\beta F=-\ln Z_{1 b}-\beta E_{M F}^{i n t}$ which is nothing but equation (23).

\subsection{Implications for the liquid-gas transition}

We have seen in Section 2.3 (Chap. 1) that the isomorphism between the Ising model and the lattice gas model implies that all physical results concerning magnetic transitions can be easily translated in the fluid language, and applied within minor modifications to the liquid-gas transition. To this aim the Ising canonical partition sum $Z^{\text {can }}$, or free energy $F=-\beta^{-1} \ln Z^{\text {can }}$, has to be transformed into the lattice gas grand-canonical partition sum $Z^{G C}$, or grand potential $\Omega=-\beta^{-1} \ln Z^{G C}$. If we only focus on the interaction part of the lattice las model, this leads to

$$
\exp \left(-\beta \Omega_{L G}\right)=\exp \left(-\beta F_{I S}\right) \exp \left(\beta N\left(\frac{J q}{2}+\frac{\mu}{2}\right)\right)
$$

In the mean field approximation $F_{I S}=-\beta^{-1} \ln Z_{M F}$ is given by equation (19), giving for the lattice gas grand potential

$$
\frac{\Omega_{L G}}{N}=\frac{J q}{2} m^{2}-\frac{1}{\beta} \ln (2 \operatorname{ch}(\beta(h+J q m)))-\frac{J q}{2}-\frac{\mu}{2} .
$$

The total number of lattice sites in the lattice gas framework represents the volume of the fluid $N=V$. The equation of state $p=\beta^{-1} \partial_{V} \ln Z$ allows to access the pressure

$$
p_{L G}=-\frac{\Omega_{L G}}{V}=-\varepsilon q \rho^{2}+\beta^{-1} \ln \frac{1}{1-\rho^{\prime}},
$$

where the last equality is obtained using the magnetization equation of state (Eq. (20)) and the substitutions $m=2 \rho-1, J=\varepsilon / 2$. Figure 3 shows some selected isotherms of the fluid equation of state. At subcritical temperatures $T<T_{c}$ equation (24) predicts a backbending, which reflects the instability of the homogeneous mean field solution respect to the separation into distinct phases as in Figure 1 above. Once again, if a linear interpolation of the liquid and gas volume solutions is imposed, the usual plateau of the Maxwell construction is recovered (Fig. 3). The critical point is defined as the ending point of the coexistence zone, i.e. the point at which the first as well as the second derivative of the equation of state are zero. Substituting in equation (24) we get $\rho_{c}=1 / 2, T_{c}=J q, p_{c}=T_{c}(\ln 2-1 / 2)$. 


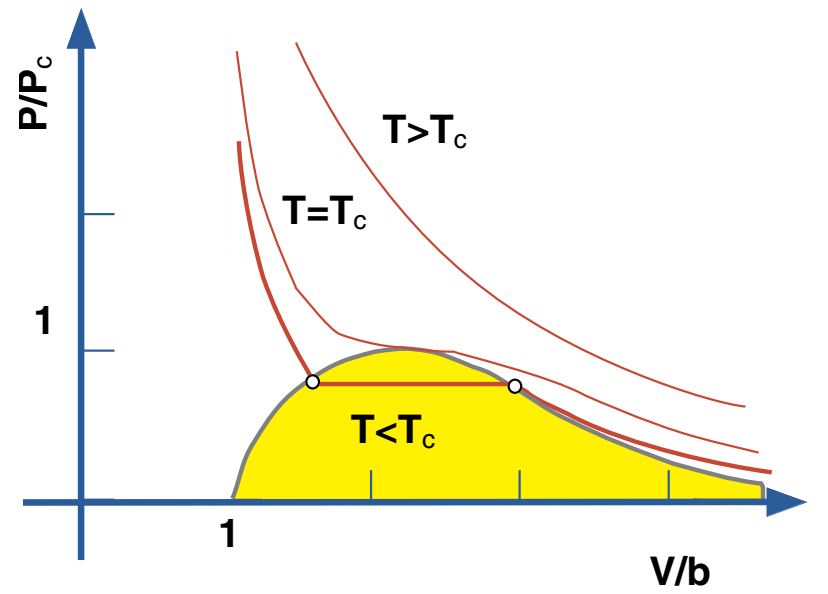

Figure 3. Isotherms in the pressure versus volume (in cell units $b$ ) plane for the threedimensional lattice gas model in the mean field approximation with a Maxwell construction of the mixed phase. The coexistence zone is also indicated.

\subsection{Metropolis simulations}

The exact solution of three dimensional Ising-based models can only be achieved through numerical simulations. The basic principle of a standard Metropolis simulation can be summarized as follows. Let us consider for simplicity a single Lagrange multiplier (i.e. the inverse of a temperature $\beta$ ) with its associated extensive variable (i.e. an energy $E$ ). An exact sampling of the partition sum $Z_{\beta}=\sum_{n} \exp \left(-\beta E^{(n)}\right)$ requires the different lattice configurations (or microstates) $n$ to be sampled with the probability $p_{n}=\exp \left(-\beta E^{(n)}\right) / Z_{\beta}$, where $E^{(n)}$ is the expectation value of the corresponding operator on the state $(n)$, here the total energy of the lattice. Starting with an arbitrary configuration (1) of the spins $s_{i}, i=1, \ldots, N_{\text {tot }}$, a second configuration (2) is generated by flipping randomly one of the $N_{\text {tot }}$ spins of the lattice. If configuration (2) does not violate a conservation law (total mass conservation if we work in the canonical ensemble), it is accepted according to the weight

$$
\frac{p_{(2)}}{p_{(1)}}=\exp \left(-\beta\left(E^{(2)}-E^{(1)}\right)\right)
$$

The thermodynamics of the model can then be calculated from a direct evaluation of the partition sum $Z_{\beta}$ via an iterative procedure [7]). At a temperature $T=\beta^{-1}$, the number of sampled realizations of the system with an energy $E$ is

$$
N(E)=N \frac{1}{Z_{\beta}} W(E) e^{-\beta E},
$$

where $W(E)$ is the degeneracy of the state and $N$ the total number of sampled states. An example for a cubic three dimensional lattice gas model in the canonical ensemble with periodic boundary conditions is given in the left part of Figure 4 [8]. From the comparison of the occupation probabilities at two different 

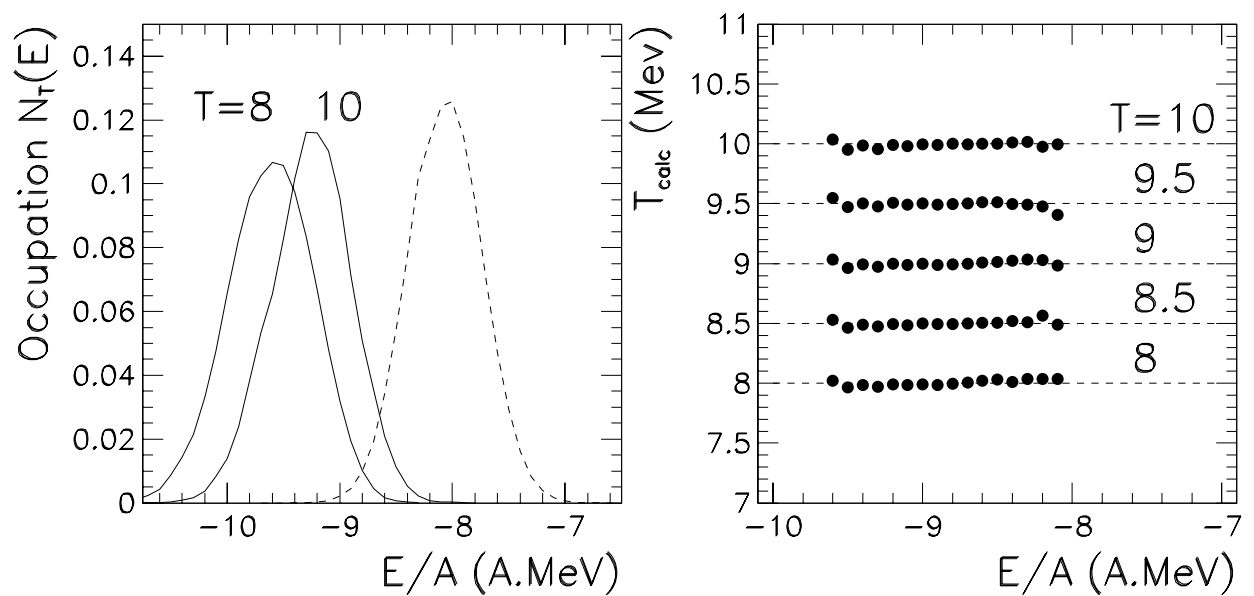

Figure 4. Left: distributions of the canonical events at two temperatures (full lines) as a function of the energy for a cubic lattice of size $L=6$ compared to a random sampling (dashed line). Right: estimation of the temperature from equation (27) (see text). The coupling constant $\epsilon$ has been fixed to $5.5 \mathrm{MeV}$.

temperatures $T_{1}, T_{2}$ one then has

$$
Z_{1}=Z_{2} \frac{N_{1}(E)}{N_{2}(E)} e^{-E\left(\beta_{2}-\beta_{1}\right)} \equiv z_{1}(E)
$$

which is valid for all the different energy bins.

In order to profit from all the available data, we can compute the partition sum as a weighted average of the above relation over the various energy bins

$$
Z_{1}=\frac{\sum_{E} z_{1}(E) \sqrt{N_{2}(E) N_{1}(E)}}{\sum_{E} \sqrt{N_{2}(E) N_{1}(E)}}
$$

Then, $Z_{1}$ is obtained iteratively with an initial normalization to the infinite temperature limit where the partition sum is analytical. Indeed if we have $A$ particles and $M$ lattice sites (canonical ensemble), the $\beta=0$ partition sum is simply

$$
Z_{0}=\sum_{E} W(E)=\frac{M !}{A !(M-A) !}
$$

A check of the precision of this method is shown in Figure 4: once the partition sum is known, equation (25) can be inverted leading to an a posteriori estimation of the temperature

$$
T^{-1}=\frac{1}{E}\left(\ln Z_{0}-\ln Z_{\beta}-\ln \frac{N_{\beta}(E)}{N_{0}(E)}\right) \equiv \beta_{\text {calc }}(E),
$$


via a comparison (presented in the left part of Fig. 4) of the occupation $N_{\beta}(E)$ at temperature $\beta^{-1}$ with the occupation in the infinite temperature limit $N_{0}(E)$, which is given by a random sampling. Equation (27) is again valid for all energy bins. The fact that the calculated temperature $\beta_{\text {calc }}^{-1}=T_{\text {calc }}$ for each energy bin is independent of $E$ is a strong test of the numerical sampling of the canonical ensemble, and is demonstrated in the right part of Figure 4. Moreover the fact that the a posteriori estimation of the temperature $T_{\text {calc }}$ is statistically consistent with the input one $T$, shows the precision of the calculation of the partition sum that can be achieved with the Metropolis method.

\section{Finite systems: getting more from pushing less}

In the preceding sections we have defined a first order phase transition as a discontinuity in the first derivative (or order parameter) $m$ of the thermodynamic potential $F$ as a function of the control parameter. Such a discontinuity can exist only in the thermodynamic limit since

- this discontinuity corresponds to phase coexistence according to the equation (see Sect. 2.2, Chap. 1)

$$
F\left(\alpha m_{1}+(1-\alpha) m_{2}\right)=\alpha F\left(m_{1}\right)+(1-\alpha) F\left(m_{2}\right),
$$

which holds if the free energy per particle is independent of the number of particles, i.e. if surface can be neglected respect to volume, which is only possible if the volume goes to infinity;

- if the system is finite the partition sum is a sum over a finite number of configurations, i.e. an analytic function. As a consequence, its first order derivative $\langle m\rangle$ according to the corresponding equation of state cannot present discontinuities.

For these reasons, it is often stated that phase transitions are only defined for infinite systems. Following this viewpoint, finite systems can present only smooth phase changes (cross-overs); to demonstrate the asymptotic existence of a phase transition a careful study of the behaviour of thermostatistical variables with the size of the system (finite size scaling [9]) has to be performed; in this context, finite size scaling is also the only way to determine the order of a transition if one believes that in finite systems all transitions are smooth.

These considerations are based on analyses where the intensive variable associated with the order parameter is controlled (i.e. the order parameter is only measured in average). In such a case, indeed the equation of state are always smooth in finite system. But what happens in other statistical ensembles?

A fundamental theorem in statistical mechanics, the Van Hove theorem, demonstrated in the next section, guarantees the equivalence between different statistical ensembles at the thermodynamic limit. However, it tells nothing about finite systems except that the theorem cannot be demonstrated. If this equivalence 
between statistical ensembles would be a good approximation even for finite systems, an experiment where the order parameter is controlled event by event (e.g. for the Ising model all events in the statistical sample share the same value of the magnetization) would lead to the same equation of state as the one obtained when the conjugated field is imposed to the system, but the magnetization remains free to fluctuate. Then the sudden jump of the magnetization observed in infinite systems would be replaced by a smooth variation. If this would be true, the existence of the transition in the finite system could only be proved through finite size scaling techniques.

This is in fact not the case.

In the next sections we will show that the Van Hove theorem is violated in first order phase transitions if the system is finite, and this violation can persist up to the thermodynamic limit in the case of long range forces. A consequence of that is that it will be possible to give a rigorous definition of phase transitions even in finite systems, with the prediction (and in some case the experimental evidence, see Chap. 2) of fancy phenomena as negative heat capacities, negative compressibilities and negative susceptibilities.

The non-equivalence of statistical ensembles has also important conceptual consequences. It implies that the value of thermodynamic variables for the very same system depends on the type of experiment which is performed (i.e. on the ensemble of constraints which are put on the system), contrary to the standard thermodynamic viewpoint that water heated in a kettle is the same as water put in an oven. This point will be discussed in Section 6 (Chap. 1).

\subsection{The Van Hove theorem}

Let us consider a classical system of distinguishable particles in a volume $V$. Let us divide $V=m V_{0}+V_{1}$ in $m$ equal boxes separated by a "corridor" of width $b$ larger than the range of the force such that the interactions among particles in different boxes can be neglected, (see Fig. 5). Let us calculate the grand
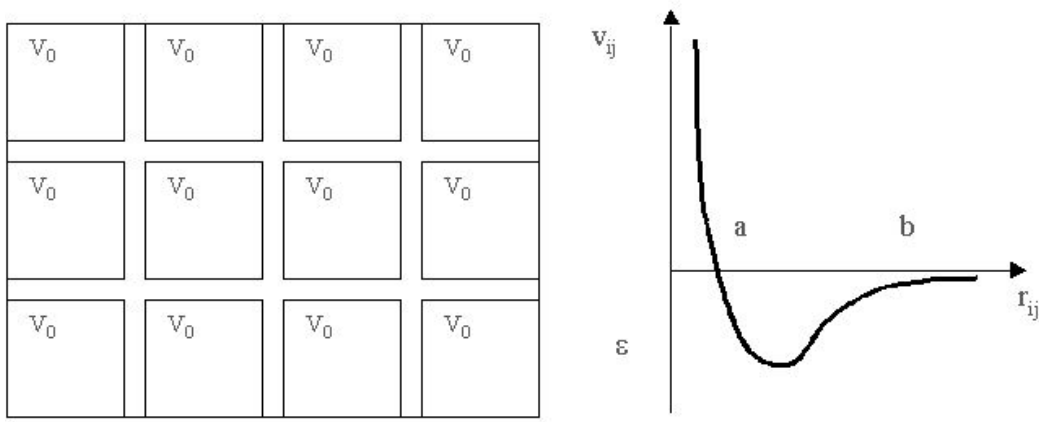

Figure 5. Schematic representation of the Van Hove theorem demonstration (left) and the corresponding inter-particle interaction (right). 
potential $\Omega=-T \ln Z$

$$
Z_{\beta \mu}(V)=\sum_{n} \exp \left(-\beta\left(H^{(n)}-\mu N^{(n)}\right)\right),
$$

where the sum extends over all the possible configurations of the system. Here $H^{(n)}=K^{(n)}+U^{(n)}\left(N^{(n)}\right)$ represents the energy (number of particles) of the system in the configuration $(n)$, and $\beta, \mu$ are the associated Lagrange multipliers, the inverse temperature and the chemical potential respectively. The partition sum results

$$
Z_{\beta \mu}(V)=\sum_{N=0}^{\infty} \alpha^{N} z_{k}^{N} \frac{1}{N !} \int_{V} d^{3 N} r \exp (-\beta U)=\sum_{N=0}^{\infty} \alpha^{N} z_{k}^{N} Z_{\beta}(N, V),
$$

with $\alpha=\exp (\beta \mu), z_{k}=\left(2 m \pi / h^{2} \beta\right)^{3 / 2}$ and the canonical partition sum $Z_{\beta}(N, V)=$ $(1 / N !) \int_{V} d^{3 N} r \exp (-\beta U)$. In the thermodynamic limit $N \rightarrow \infty, V \rightarrow \infty, V_{0} \rightarrow \infty$, $V / N \rightarrow v=c t e$, the volume of the corridor $V_{1} \propto m V_{0}^{2 / 3}$ can be neglected respect to the total volume. The contribution to the partition sum of the particles in the corridor can be neglected too if the interaction is short range, such that only a finite number of particles $(b / a)^{3}$ can interact with a given particle. Then if we define the auxiliary partition sums $\tilde{Z}_{\beta}(N, V)$ and $\tilde{Z}_{\beta \mu}(V)$, obtained under the constraint that no particle is in the corridor, it is easy to demonstrate [10] that asymptotically

$$
\begin{aligned}
Z_{\beta \mu}(V) \underset{V}{\longrightarrow} \tilde{Z}_{\beta \mu}(V) \\
Z_{\beta}(N, V) \underset{V}{\longrightarrow} \tilde{Z}_{\beta}(N, V) .
\end{aligned}
$$

Using once again the fact that the force is short ranged, the grancanonical partition sum can be factorized as $\tilde{Z}_{\beta \mu}(V)=\left(Z_{\beta \mu}\left(V_{0}\right)\right)^{m}$ giving

$$
\frac{\ln Z_{\beta \mu}(V)}{V} \underset{V \rightarrow \infty}{\longrightarrow} \frac{\ln Z_{\beta \mu}\left(V_{0}\right)}{V_{0}}
$$

and a similar relation can be established [10] in the canonical case

$$
\frac{\ln Z_{\beta}(N, V)}{N}=f_{N}\left(\frac{V}{N}\right) \underset{N \rightarrow \infty}{\longrightarrow} f(v) .
$$

This demonstrates that a thermodynamic limit exists for these systems. Indeed if the thermodynamic potential per unit volume tends to a constant independent of 
the volume (or the number of particles), the observables distributions $P_{N}(A)$ will also tend to an asymptotic function

$$
\lim _{N \rightarrow \infty} P_{N}(A)=\tilde{p}\left(\frac{A}{N}\right)^{N} .
$$

Equation (29) also implies that in the thermodynamic limit ensembles are equivalent. Indeed if $\omega=Z_{\beta \mu}\left(V_{i}\right) / V_{i}$ for an arbitrary subsystem $V_{i}$, this implies that reduced extensive variables are intensive, i.e. that the asymptotic distribution $\widetilde{p}(A / N)^{N}$ has a vanishing width in agreement with the central limit theorem (Sect. 2.2, Chap. 1). Since ensembles differ at the level of fluctuations, this shows the equivalence between ensembles.

This equivalence can be demonstrated explicitly by showing that the equation of state which is defined both in the grancanonical and in the canonical ensemble, namely $p=-\partial_{V} \ln Z$, is the same in the two ensembles [10]. Indeed let us consider a single box containing $n$ particles. The auxiliary partition sum can be written as

$$
\tilde{Z}_{\beta}(N, V)=\sum_{n=0}^{N} Z_{\beta}\left(n, V_{0}\right) \tilde{Z}_{\beta}\left(N-n, V-V_{0}\right) .
$$

In the limiting situation in which $\tilde{Z}_{\beta}$ can be confused with $Z_{\beta}$ we can write

$$
\begin{aligned}
\ln Z_{\beta}\left(N-n, V-V_{0}\right) & =(N-n) f_{N}\left(\frac{V-V_{0}}{N-n}\right) \\
& \approx(N-n) f_{N}\left(\frac{V}{N}\right)+\left(n \frac{V}{N}-V_{0}\right) \partial_{v} f_{N}\left(\frac{V}{N}\right),
\end{aligned}
$$

where the expansion to the first order in $n$ is justified by the short range repulsion of the interaction which guarantees that, if the volume $V_{0}$ is let to infinity slower than the total volume $V, n$ is asymptotically small respect to $N$. Replacing this expression in equation (30) we get

$$
\begin{aligned}
\tilde{Z}_{\beta}(N, V) & =\exp \left(N f_{N}(v)\right) \\
& \approx \exp \left(N f_{N}-V_{0} \partial_{v} f_{N}\right) \sum_{n=0}^{\infty} Z_{\beta}\left(n, V_{0}\right) \exp \left(n\left(v \partial_{v}-1\right) f_{N}\right),
\end{aligned}
$$

which gives an implicit equation for $\partial_{v} f_{N}$

$$
\exp \left(V_{0} \partial_{v} f_{N}\right) \approx \sum_{n=0}^{\infty} Z_{\beta}\left(n, V_{0}\right) \exp \left(n\left(v \partial_{v}-1\right) f_{N}\right),
$$

and finally the equation of state

$$
\frac{\partial \ln Z_{\beta}(N, V)}{\partial V}=\frac{\partial f_{N}(v)}{\partial v}=\frac{\ln \sum_{n=0}^{\infty} \alpha^{n} Z_{\beta}\left(n, V_{0}\right)}{V_{0}}=\frac{\partial \ln Z_{\beta \mu}(V)}{\partial V} .
$$


This demonstrates the equivalence of the equation of state for all values of the extensive variable $V$.

An additional problem arises in first order phase transitions, since in this case the coexistence region is not accessible to the intensive ensemble, and therefore the right hand side of equation (31) is not defined.

In this case one may wonder whether the ensemble inequivalence may be preserved up to the thermodynamic limit. We now show that this is not the case, if the forces are short ranged.

Explicitly let us show that, at the thermodynamic limit, the jump in the extensive variable observed at the transition point of the intensive ensemble (here grancanonical) must correspond to a plateau in the corresponding extensive ensemble (here canonical). We have just demonstrated (Eq. (31)) that in the thermodynamic limit a canonical ensemble (with partition sum $Z_{\beta}(N, V)$ ) can be seen as a collection of a number $m$, eventually going to infinity, of grancanonical ensembles (with partition sum $Z_{\beta \mu}\left(V_{0}\right)$ ) at equilibrium, i.e. characterized by the same temperature, pressure and chemical potential. Indeed for a given box $i$ of volume $V_{0}$ the ensemble of the other $m-1$ boxes can be seen as a particle reservoir (recall $V_{0}$ goes to infinity slower than $V$ ), leading to a fluctuating number of particles in the $i$ th box under the constraint of the global conservation law $\sum_{i=1}^{m} N_{i}=N$. Let us consider for the global canonical system a density value $\rho_{t}=N / V$ lying inside the coexistence region. This value is inaccessible to the grancanonical ensemble for which the density jumps at the transition pressure from the ordered phase value $\rho_{L}=N_{L} / V_{0}$ to the disordered value $\rho_{G}=N_{G} / V_{0}$. The only possibility to get a total density between $\rho_{L}$ and $\rho_{G}$ combining grancanonical systems at the transition pressure and respect the conservation laws, is to mix the pure states $\rho_{L}$ and $\rho_{G}$ in linear proportion, i.e. to perform a Maxwell construction.

This shows that the straight line construction in Figure 3 is the physical behaviour expected for a first order phase transition in the thermodynamic limit also if the total number of particles is controlled. This completes our demonstration of the asymptotic equivalence between statistical ensembles.

It is important to stress that the thermodynamic limit is essential throughout the whole demonstration, and therefore the equivalence is not guaranteed for finite systems. In the next section we will show that the violation of the Van Hove theorem in finite systems leads to the emergence of new thermodynamic phenomena in first order phase transitions of mesoscopic systems.

\subsection{Convexity anomalies and phase transitions}

Let us consider the thermodynamics of a first order phase transition in an extensive ensemble, i.e. in the physical case where the order parameter is a controlled variable.

A schematic representation of the liquid gas phase transition in the canonical ensemble is given in Figure 6. Here the order parameter is the particle density $\rho=A_{0} / V$. The mean field solution for the free energy $f=F\left(A_{0}, V\right) / A_{0}$ (upper left, dashed line) at a given temperature shows two minima in the spirit of Landau 

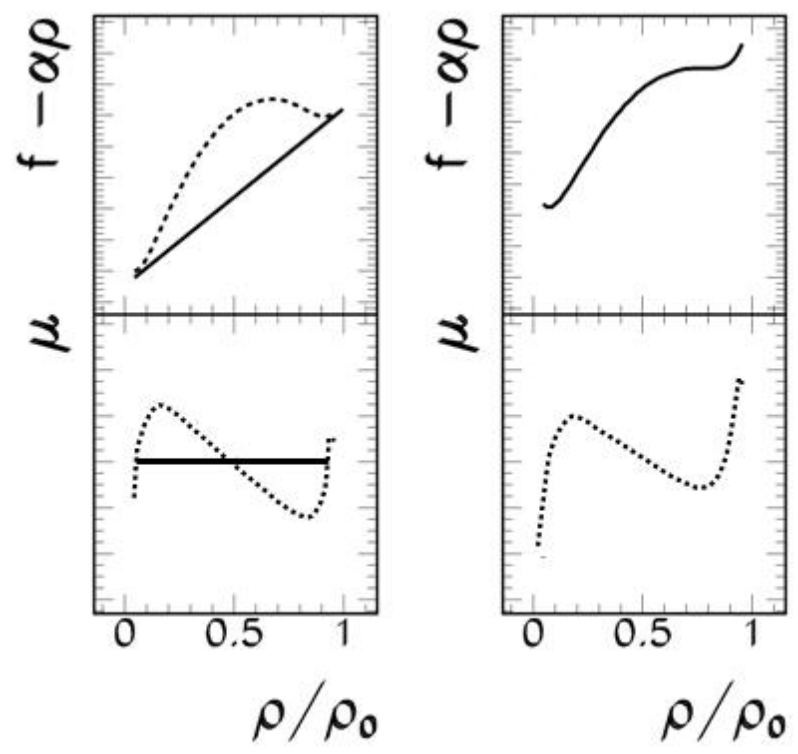

Figure 6. Schematic representation of the liquid-gas phase transition in the canonical ensemble for an infinite system (left side) and a finite system (right side). The physical result is given by the full lines. Dashed lines: mean field approximation.

theory, corresponding to a gas-like solution at low density and a liquid-like solution at high density. A tangent construction (upper left, full line) corresponds to the inclusion in the partition sum of mixed partitions, given by linear combinations of the two solutions. This linear interpolation is only possible in infinite systems for which we can neglect the role of the interface (the "corridor" between the two phases); this leads to a plateau in the conjugated intensive variable $\mu=\partial F / \partial A_{0}$ (left part, lower panel). If the system is finite (right part of Fig. 6), the free energy per particle of the liquid fraction is higher than in the case of the pure liquid solution because of the increased surface tension; as a result the free energy of the mixed configuration (upper right) is higher than the tangent construction, i.e. is a concave function of $\rho$, giving rise to a backbending in the chemical potential (lower right) and a negative susceptibility $\chi^{-1}=\partial_{A_{0}} \mu$.

This intuitive reasoning is demonstrated for the lattice gas model in Figures 7 and 8 that show the free energy, chemical potential and pressure obtained from a canonical three dimensional Monte Carlo simulation for a linear dimension $L=6$ and different values of the temperature $[8,11]$. The different quantities are calculated as numerical derivatives of the canonical partition sum that is evaluated from the iterative procedure (Eq. (26)). The convexity anomaly of the partition sum is observed in the particle density $\rho=A_{0} / L^{3}$ direction which represents the order parameter of the transition; $\rho$ can be seen both as a variable number of particles at fixed volume or as an inverse number of lattice sites at fixed number of particles. For this reason the same anomalous backbending presented 


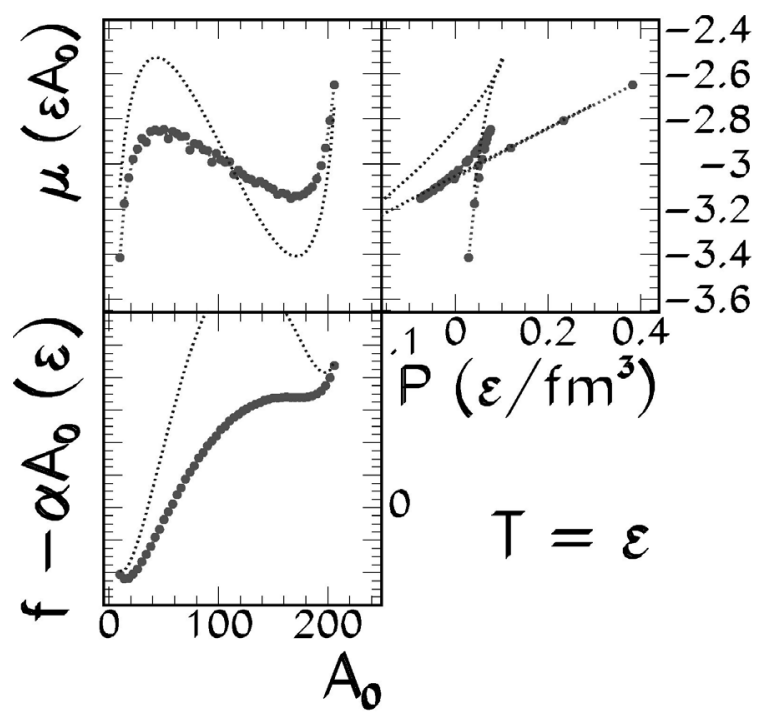

Figure 7. Free energy, chemical potential and pressure for a $6 \times 6 \times 6$ lattice gas at a subcritical temperature. Dashed lines: mean field approximation; symbols: exact results.

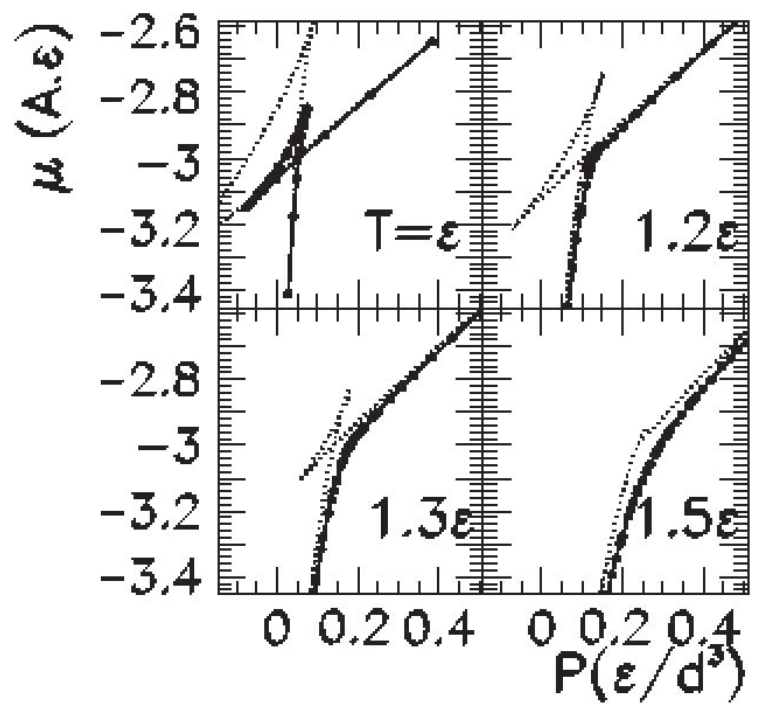

Figure 8. Chemical potential as a function of pressure at different temperatures for a $6 \times 6 \times 6$ lattice gas. Dashed lines: mean field approximation; symbols: exact results. 


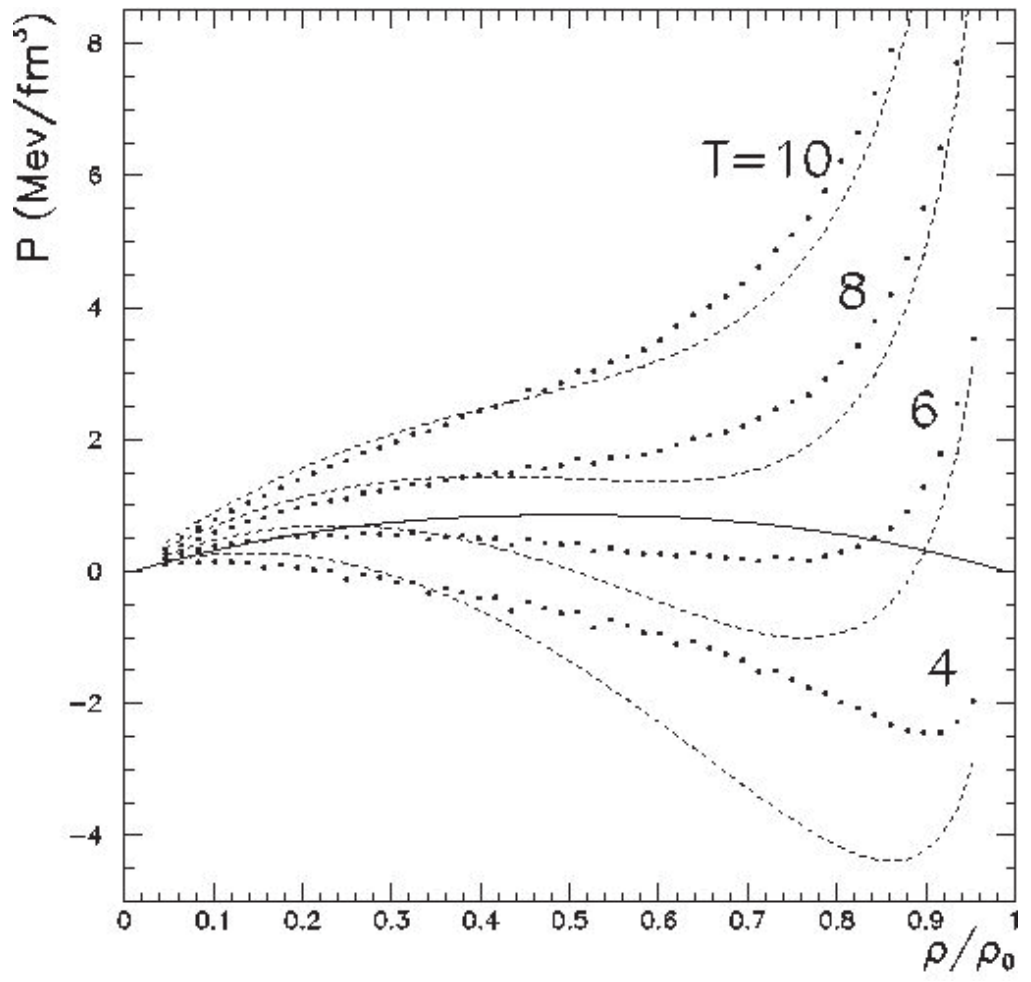

Figure 9. Pressure versus density equations of state for a $6 \times 6 \times 6$ lattice gas exactly (symbols) and in the mean field approximation (dashed lines). Solid line: coexistence curve obtained from the loop of the $\mu(p)$ diagrams of Figure 8. The coupling constant $\epsilon$ has been fixed to $5.5 \mathrm{MeV}$.

by the chemical potential is observed in the $p(V)$ equation of state as shown in Figure 9. All isotherms up to the critical temperature backbend, i.e. show a region of negative compressibility. In Figures 7, 8 and 9 the dashed lines represent the mean field approximation discussed in Section 3 (Chap. 1). Not surprisingly, the mean field badly fails in the phase transition region, while it is close to the exact result for supercritical temperatures. In particular the Van der Waals loop is still present (see Fig. 8) at temperatures at which the two phases have merged into one in the exact calculation. This means that the critical temperature is overestimated by the mean field approximation because of its intrinsic lack of fluctuations.

It is important to stress that the physical origin of the backbendings and loops is completely different in the exact calculation and in the mean field. In the former the system inside coexistence presents inhomogeneous partitions that are stable equilibrium solutions with negative compressibility and susceptibility, while in the latter the backbending reflects the instability of the homogeneous mean field solutions respect to phase separation (tangent constructions). 


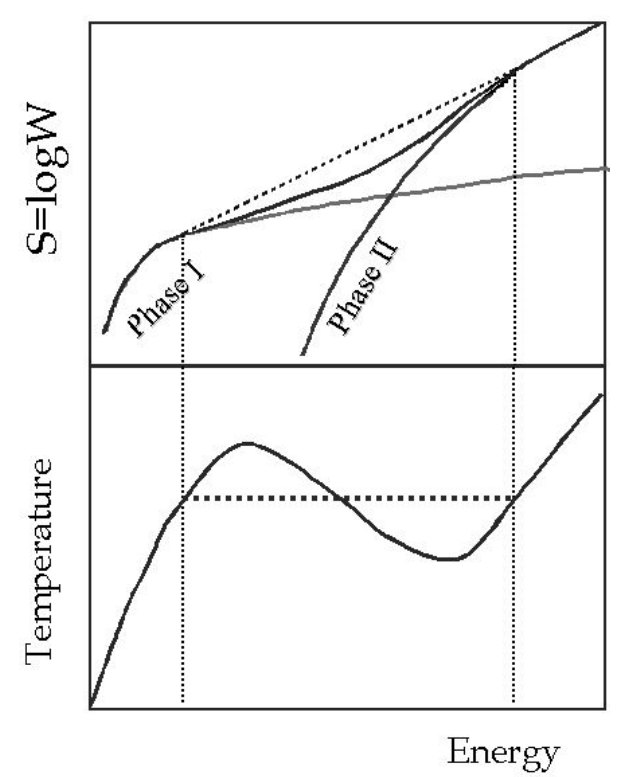

Figure 10. Schematic representation of a first order phase transition in the microcanonical ensemble.

The same reasoning as in Figure 6 can be done for the microcanonical ensemble as schematically shown in Figure $10[12,13]$. If energy is controlled, the appropriate thermostatistical potential is the microcanonical entropy. A first order phase transition can be viewed as the sudden opening of a new disordered phase at a certain threshold energy. The number of states of the disordered phase grows much faster with energy than the one associated to the ordered phase, and this creates a convex intruder in the total microcanonical entropy. This convexity anomaly cannot be cured by a conventional tangent construction because of the non negligible surface entropy at the interface between the two phases. This phenomenon is in fact observed in a Monte Carlo simulation of the lattice gas model in the isobar microcanonical ensemble as shown in Figure $11[14,15]$. The convex intruder implies a backbending in the temperature $T^{-1}=\partial_{E} S$ and a negative branch for the heat capacity $C^{-1}=\partial_{E} T$ between two divergences. Density being the order parameter of the liquid gas phase transition, since the number of particles is fixed in this calculation $\left(A_{0}=216\right)$, the volume has to increase with energy to allow the system explore the partitions belonging to the disordered phase. To this aim, the calculation shown in Figure 11 are performed at constant pressure, where pressure is here defined as the Lagrange multiplier conjugated to the system volume. In this calculation the system volume is assumed to be the average cubic radius (see Sects. 6.4, Chap. 1 and 1.2, Chap. 2). The corresponding caloric curves at constant pressure and constant volume are shown in Figure 12. 


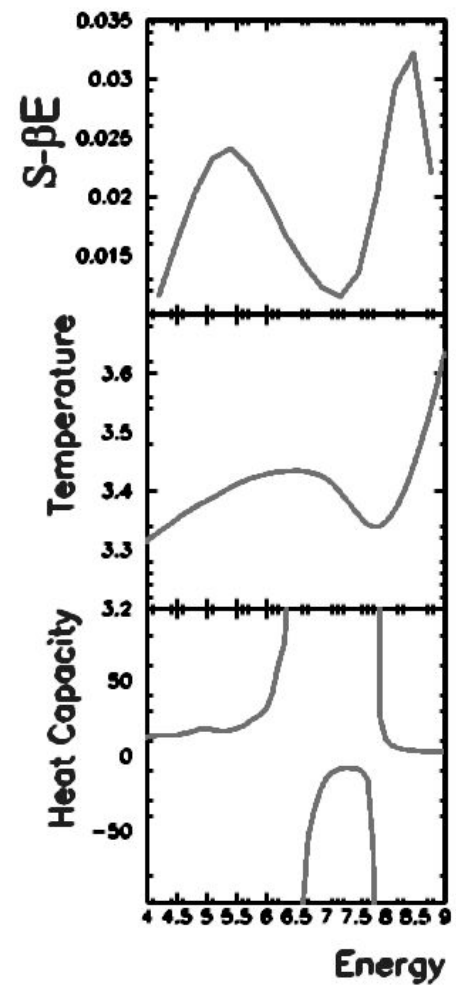

Figure 11. Entropy, temperature and heat capacity for 216 particles in the microcanonical lattice gas model at a subcritical pressure. The coupling $\epsilon$ is fixed to $5.5 \mathrm{MeV}$.

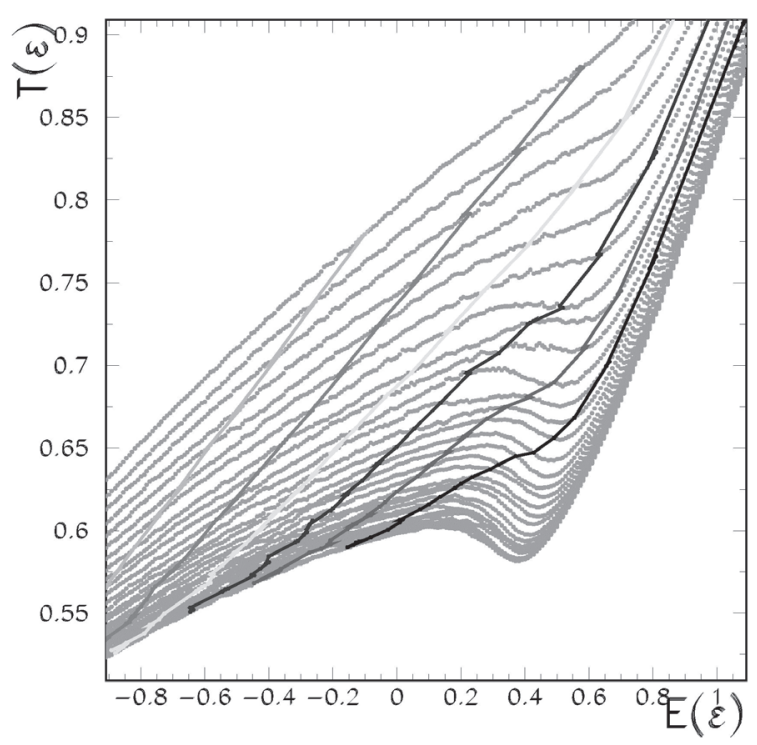

Figure 12. Caloric curves at constant pressure (showing a backbending up to the critical pressure) and at constant volume (monotonically increasing) for 216 particles in the microcanonical lattice gas model.

The isobaric curves show a backbending up to the critical pressure giving a clear definition of the coexistence zone.

To summarize, we have shown through some selected examples that a first order phase transition in a finite system is associated to a convexity anomaly in the appropriate thermostatistical potential; the direction of the anomaly in the space of observables can be defined as the order parameter of the transition and the conjugated intensive variable shows a backbending in the coexistence zone; energetic considerations confirm that this backbending transforms into a plateau (standard first order phase transition) if surfaces are negligible in the global energetic of the infinite system in agreement with the Van Hove theorem.

In particular in the microcanonical ensemble adding energy to the system can cause its temperature to decrease, and we have intuitively associated the observation of negative heat capacity with the sudden opening of a disordered collective channel [16]. To clarify the link between channel openings, phase 
transitions and negative heat capacities, in the next sections we will consider some simple analytical equilibrium models that exhibit negative heat capacity.

\subsection{Interacting particles in harmonic potentials: the classical case}

To see the link between the opening of a channel and negative heat capacity, let us consider the simple example of $A$ classical particles in two harmonic oscillators of different frequency $\omega_{1}, \omega_{2}$ such that the particles in the first oscillator interact all with a constant coupling $\varepsilon$, while they are free in the second one. This model can be seen as a schematic representation of the liquid-gas phase transition. The Hamiltonian reads

$$
H=\sum_{i=1,2} \sum_{k=1}^{A_{i}} \frac{p_{k}^{2}}{2 m}+\frac{1}{2} k_{i} x_{k}^{2}+\varepsilon A_{1}\left(A_{1}-1\right)
$$

where $A_{i}$ is the number of particles in the $i$ th oscillator. The entropy of such a system is exactly calculable. Indeed the number of states with energy $e \leq E$ accessible to a particle in an harmonic oscillator with frequency $\omega=\sqrt{k / m}$ is

$$
\Omega(E)=\int_{\sigma(E)} \frac{d x d p}{h}=\frac{2 \pi E}{h \omega},
$$

where $\sigma(E)$ is the surface in phase space defined by the iso-energy curve $p^{2} / 2 m+$ $k x^{2} / 2=E$. Similarly the number of states with energy $e \leq E$ accessible to $A$ particles will be given by

$$
\Omega_{A}(E)=\left(\frac{2}{h \omega}\right)^{A} V_{2 A}(\sqrt{E}),
$$

where $V_{n}(x)$ is the volume of an hypersphere in $n$ dimensions with radius $x$. The state density $W(E)=d \Omega / d E$ results

$$
W_{A}^{\omega}(E)=\left(\frac{2 \pi}{h \omega}\right)^{A} \frac{E^{A-1}}{(A-1) !} .
$$

This formula can be generalized to the case of $A_{1}\left(A_{2}\right)$ particles in the oscillator at frequency $\omega_{1}\left(\omega_{2}\right)$ such that the total sum gives $A$, i.e. the case (32) with no interaction, $\varepsilon=0$ :

$$
\begin{aligned}
\Omega_{A}(E) & =\sum_{A_{1}=0}^{A} \frac{A !}{A_{1} !\left(A-A_{1}\right) !}\left(\frac{2}{h \omega_{1}}\right)^{A_{1}}\left(\frac{2}{h \omega_{2}}\right)^{A-A_{1}} V_{2 A}(\sqrt{E}) \\
W_{A}(E) & =\left(\frac{2 \pi}{h}\right)^{A} \frac{E^{A-1}}{(A-1) !}\left(\frac{1}{\omega_{1}}+\frac{1}{\omega_{2}}\right)^{A},
\end{aligned}
$$


which is equivalent to a unique oscillator at an effective frequency, $\omega_{\text {ef }}^{-1}=\omega_{1}^{-1}+\omega_{2}^{-1}$. The entropy of this model $S(E) \propto \ln (E)$ is a regular function of positive concavity. Equation (33) shows that the possible existence of the system in two configurations of different density does not imply a phase transition. However if we introduce an interaction in the first oscillator $(\varepsilon \neq 0)$, the situation drastically changes. Indeed in this case to have a total energy $E$, the energy of the $A_{1}+A_{2}$ independent particles that enters the hypersphere volume has to be $E-\varepsilon A_{1}\left(A_{1}-1\right)$ and the sum in equation (33) becomes

$$
W_{A}^{\varepsilon}(E)=\sum_{A_{1}=0}^{A}\left(\begin{array}{l}
A \\
A_{1}
\end{array}\right)\left(\frac{\omega_{1}}{\omega_{2}}\right)^{A-A_{1}} W_{A}^{\omega_{1}}\left(E-\varepsilon A_{1}\left(A_{1}-1\right)\right) .
$$

If the second oscillator is sufficiently soft, the microcanonical entropy presents a convex intruder leading to a backbending of the caloric curve and negative heat capacity as shown in Figure 13. The same anomaly observed in the density of states is present in the average kinetic energy (dashed line in Fig. 13), showing

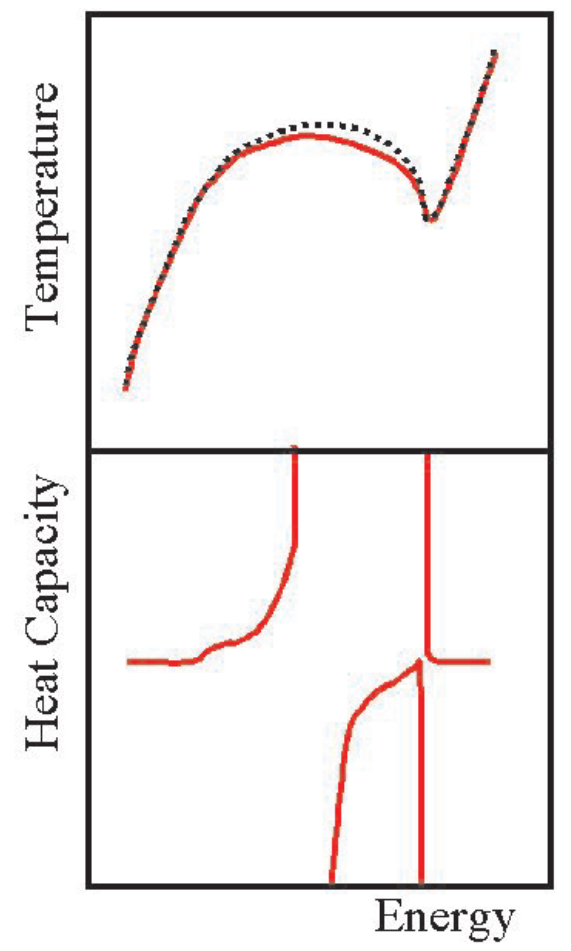

Figure 13. Temperature and heat capacity for the classical two oscillator model in the subcritical regime. Full curves: derivatives of the entropy. Dashed curve: average kinetic energy per degree of freedom. 
that the convex intruder corresponds indeed to a physical cooling of the system (see Sects. 4.7 and 7.4, Chap. 1 for a deeper discussion on this subject).

From this analytical example of the occurrence of a negative heat capacity, we can come to the following conclusions:

1. negative heat capacity is not an artifact of imperfect numerical simulations: a $C<0$ system can be thermodynamically stable;

2. the existence of two different kinds of states for a system appears as a phase coexistence only if their respective level densities are sufficiently different (for instance particles interacting only in the low energy phase $(\varepsilon>0)$ and a much higher volume ( $\omega_{1} / \omega_{2}$ large) available for the high energy phase) so that their addition presents a concavity anomaly;

3. if the average energy of the two phases is not the same (i.e. energy is an order parameter) a finite isolated system in equilibrium at phase coexistence presents a negative heat capacity.

\subsection{Interacting particles in harmonic potentials: the quantum case}

In the classical model we have studied, the presence of a back bending in the caloric curve is indisputable. However, one may worry about the generality of such a statement. Do such anomalies also exist in quantum systems, or is this definition of phase transition restricted to classical systems? In order to address this question, we can solve the quantum analog of the model of Section 4.3 (Chap. 1). Let us consider again A particles which can jump from one harmonic oscillator to another. In the first one all particles interact while in the second one they are free. The curvature of the second well plays the role of a confining potential, similar to a pressure. The corresponding Hamiltonian reads

$$
\hat{\mathbf{H}}=\omega_{1}\left(\hat{\mathbf{N}}_{1}-\frac{A_{1}}{2}\right)+\omega_{2}\left(\hat{\mathbf{N}}_{2}-\frac{A_{2}}{2}\right)-\varepsilon\left(\hat{\mathbf{A}}_{1}^{2}-A^{2}\right)
$$

with the operators

$$
\begin{aligned}
& \hat{\mathbf{N}}_{i}=\sum_{n=1}^{A} \delta_{i_{n}}^{i}\left(a_{n} a_{n}^{+}\right) \\
& \hat{\mathbf{A}}_{i}=\sum_{n=1}^{A} \delta_{i_{n}}^{i}
\end{aligned}
$$

where $i_{n}$ is the harmonic well occupied by the particle $i$, and we have neglected for simplicity the energy shift $\varepsilon \hat{\mathbf{A}}_{1}$. Using this Hamiltonian we can compute the level density and its associated entropy. To simplify the calculation we have chosen $\omega_{1}$, 


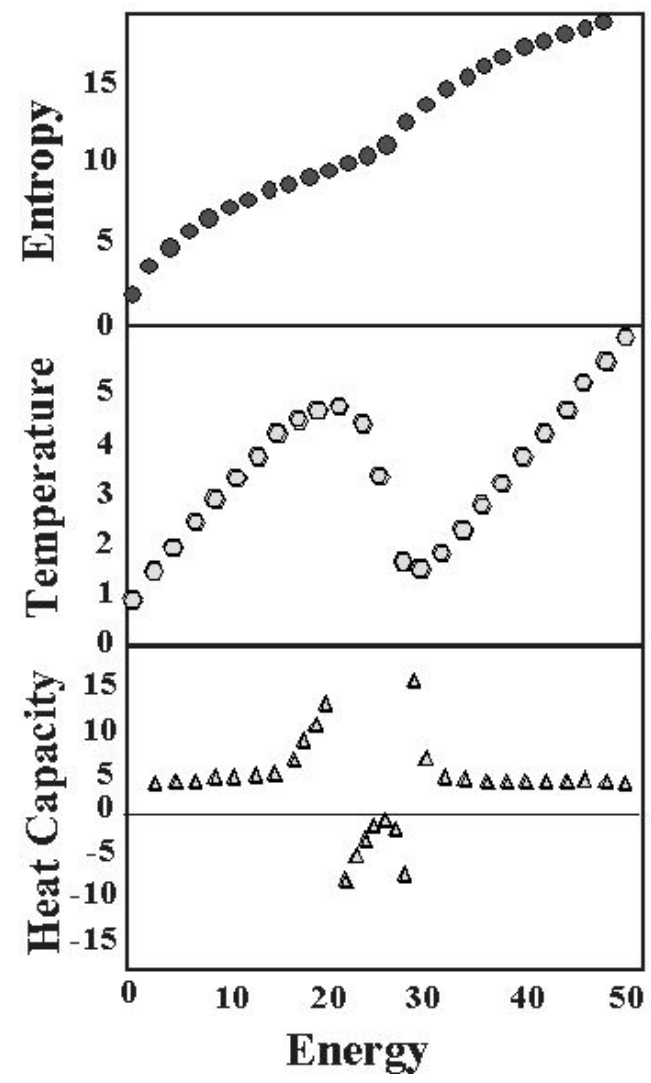

Figure 14. Microcanonical entropy, temperature and heat capacity for the two quantum oscillators model in the subcritical regime.

$\omega_{2}$ and $\varepsilon$ to be commensurable, $\omega_{2}=\omega_{1} / \lambda$ and $\varepsilon=\omega_{1} / \mu$. The energy results

$$
\frac{E}{\varepsilon_{1}}=N_{1}-\frac{A_{1}}{2}+\frac{A^{2}-A_{1}^{2}}{\mu}+\frac{N_{2}}{\lambda}-\frac{A-A_{1}}{2 \lambda} .
$$

In a harmonic oscillator the number of states associated with $N_{1}$ quanta carried by $A_{1}$ particles is $\left(\begin{array}{l}N_{1}-1 \\ A_{1}-1\end{array}\right)$ so that for the double oscillator system this corresponds to

$$
W^{A}(E)=\left(\begin{array}{l}
A \\
A_{1}
\end{array}\right)\left(\begin{array}{l}
N_{1}-1 \\
A_{1}-1
\end{array}\right)\left(\begin{array}{l}
N_{2}-1 \\
A-A_{1}-1
\end{array}\right) .
$$

Then, we can compute the temperature and the associated heat capacity (see Fig. 14). We observe that the system indeed presents an anomaly in the curvature of the entropy. Back-bending and negative heat capacities automatically follow. 


\subsection{Phase transitions versus channel openings}

In the last section we have associated a phase coexistence in the bulk, to the existence in the finite system of (at least) two kinds of states that differ in their respective level densities. If the microscopic origin of a first order phase transition can always be traced back to the opening of a new channel as a function of the order parameter (see Sect. 6.8, Chap. 1 for a formalization of this statement), it is however important to observe that not all channel openings are associated to phase transitions in the thermodynamic limit. A typical example in molecular and cluster physics is given by isomerization, that may lead to accidents in the concavity of the entropy without being connected to a phase change in the bulk [17]. In order to clarify the physics involved in the backbending of the caloric curve, let us consider the simplest possible case: the state change from one dimer to two monomers. This is a system with only two particles in a box of volume $V$, which can exist in two states:

- a bound system of mass $2 m$ bounded by an energy $-\epsilon$ with a state density

$$
W_{\text {bound }}(e)=\frac{\pi^{3 / 2}}{\left(\frac{3}{2}\right) !} \frac{V}{h^{3}}(4 m(e+\epsilon))^{3 / 2} \theta(e+\epsilon) ;
$$

- a state with two free monomers corresponding to

$$
W_{\text {free }}(e)=\frac{\pi^{3}}{3 !}\left(\frac{V}{h^{3}}(2 m e)^{3 / 2}\right)^{2} \theta(e) .
$$

The total density of states is $W_{\text {tot }}=W_{\text {bound }}+W_{\text {free }}$. As it can be seen from Figure 15, at sufficiently low density (i.e. large volume) the microcanonical entropy presents a convex intruder characteristic of a first order phase transition.

The extension of this model to a larger and eventually infinite number of constituents is straightforward. Indeed, if only two body bounds are allowed in

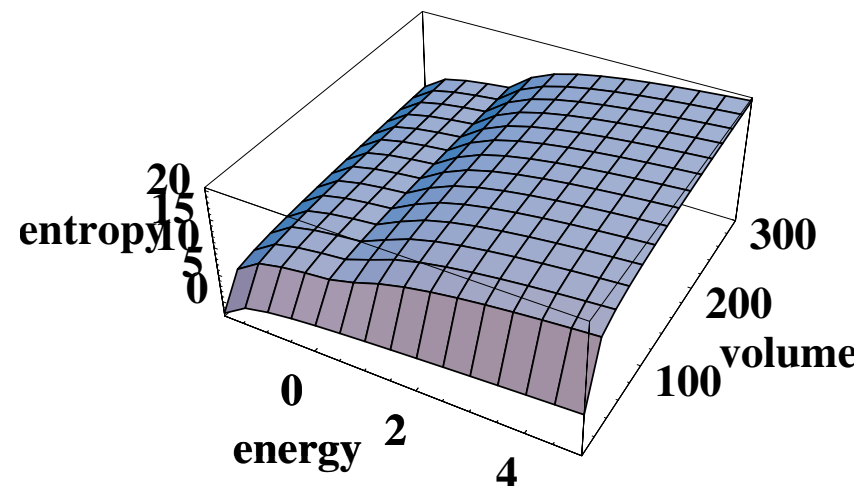

Figure 15. Entropy as a function of the volume and the total energy for the state change from one dimer to two monomers. 
a system of $N$ particles, we can write for the density of states

$$
W_{\text {tot }}(e)=\sum_{k=0}^{N / 2}\left(\begin{array}{l}
N \\
2 k
\end{array}\right)\left(\begin{array}{l}
2 k \\
2
\end{array}\right) c_{3 k} c_{3(N-2 k)} w_{m, V}^{N-2 k}(e+k \epsilon) w_{2 m, V}^{k}(e+k \epsilon) \theta(e+k \epsilon),
$$

where $c_{2 n}=\pi^{n} / n !$ and $w_{m, V}(e)=V(2 m e)^{3 / 2} / h^{3}$. As it can be seen from Figure 16, the anomaly in the entropy disappears going towards the thermodynamic limit, similarly to the ionization phenomenon which is known to present a smooth cross-over from a dimer gas to a plasma without going through a phase transition region. This behaviour is due to the fact that the distance between the successive thresholds (measured in energy per particle) is a decreasing function of the number of constituents.

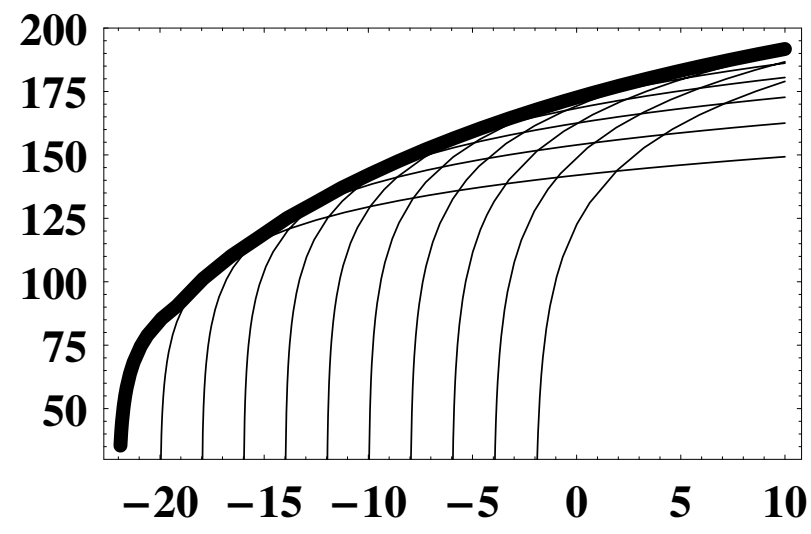

Figure 16. Entropy as a function of energy for the state change from one dimer to two monomers for $V=200$ and a number of constituents $N=$ 22. Thin lines: channels corresponding to the breaking of each pair; thick line: total en-

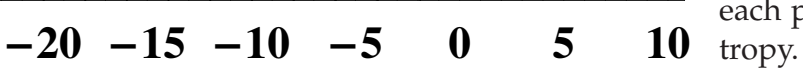

If, on the other hand, we allow many body correlations connecting together an arbitrary number of particles to form a polymer (or liquid-like state), the state change observed in the two body system would converge to a liquid gas phase transition. From this simple example we can get an intuitive understanding of the microscopic origin of phase transitions. A channel opening in a finite system corresponds to a phase transition in the bulk if

- it is sudden enough to lead to a convex intruder in the entropy,

- and the corresponding order parameter is an observable collective enough to scale linearly with the number of constituents.

A more rigorous demonstration of this point will be given in Section 6.8 (Chap. 1).

\subsection{Surface tension and negative heat capacity}

We have seen that $C<0$ is a generic feature of finite systems at coexistence, and is expected to disappear at the thermodynamic limit (at least if the interactions are short-ranged, see Sect. 5, Chap. 1). One can ask how much this behaviour is 
confined to the microscopic world: how small a system has to be, for the convex intruder to be sizeable? To answer to this question, let us consider the macroscopic analytical example of a liquid drop in equilibrium with its vapour [18].

The bulk free energy of an incompressible liquid can be parametrized in the spirit of the Landau theory as $F=F_{0}+N k\left(v-v_{0}\right)^{2} / 2$, where $k$ is the compressibility, $v$ the reduced volume $v=V / N=\rho^{-1}$ and $v_{0}$ the saturation point. For a finite liquid drop one needs to introduce an additional term coming from the surface tension $\sigma$, leading to the free energy per particle of the drop $f_{L}=f_{0}+k\left(v-v_{0}\right)^{2} / 2+\sigma s N^{-1 / 3}$, where the surface coefficient $s=S N^{-2 / 3}$. The free energy of the vapour can be analytically calculated under the approximation that the vapour is an ideal classical monatomic gas. For $N$ indistinguishable non interacting particles in a volume $V$ we have $Z=z^{N} / N$ ! with the single particle partition sum

$$
z=V \frac{1}{h^{3}} \int d^{3} p \exp \left(-\beta \frac{p^{2}}{2 m}\right)=V\left(\frac{m}{2 \pi \beta \hbar^{2}}\right)^{3 / 2},
$$

which finally gives for the free energy per particle

$$
f_{G}=-T \ln v-\frac{3}{2} T \ln T+K .
$$

The free energies of the two phases are schematically shown in Figure 17. Phase coexistence implies the equality between the two partial pressures $\partial_{v} f_{L}=\partial_{v} f_{G}$ which gives the usual tangent construction (dashed line). The finiteness of the system appears in the constraint of mass conservation. As we increase $v$ we dive

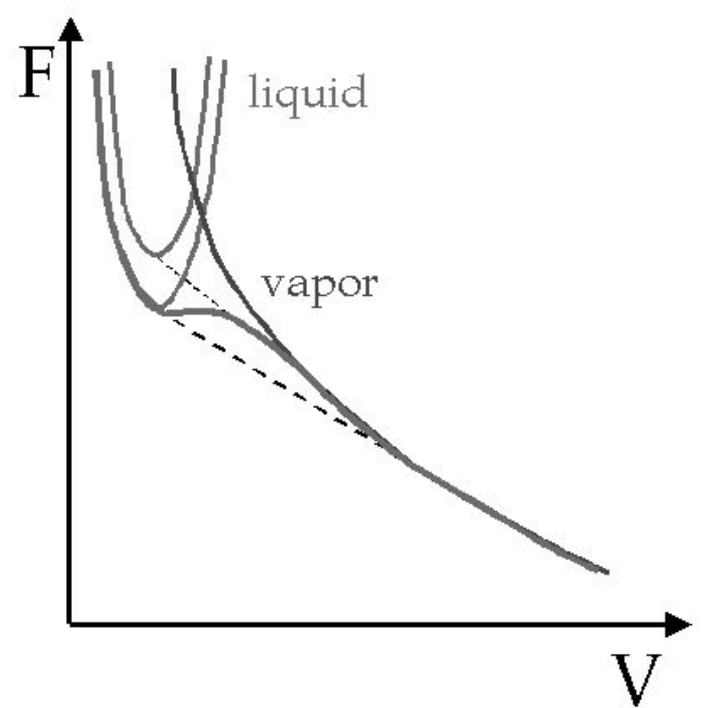

Figure 17. Schematic representation of the free energy of a liquid drop of different sizes (parabolic curves) in equilibrium with its vapour. 
inside coexistence with an increased proportion of the vapour fraction respect to the liquid fraction; this leads to an overall increase of the free energy of the drop, and a consequent increase of the slope of the tangent construction as a function of $v$. The net result is a convexity anomaly of the free energy, i.e. a negative compressibility. The backbending in the $p(v)$ equation of state can be analytically calculated using the Clapeyron equation

$$
\frac{d p}{d T}=\frac{\Delta h}{\left(v_{G}-v_{L}\right) T^{\prime}}
$$

where the enthalpy of vaporization per particle is given by $\Delta h=\Delta h_{0}-3 c_{S} v_{L} / r, c_{S}$ is the surface energy coefficient, and $r=r_{0} A^{1 / 3}$ is the drop radius. Assuming the bulk vaporization enthalpy $\Delta h_{0}$ as well as the specific volume of the drop $v_{L}$ as constant, the Clapeyron equation can be directly integrated giving

$$
p=p_{b u l k} \exp \left(\frac{3 c_{S} v_{L}}{r T}\right)
$$

which grows as the drop radius decreases (and consequently the specific volume increases), showing that the compressibility is negative at coexistence even for macroscopic droplets, while the plateau is recovered in the bulk limit. The equality between the liquid pressure (Eq. (36)) and the vapour pressure $p=T / v_{G}$ gives

$$
p_{0} \exp \left(-\frac{\Delta h_{0}}{T}+\frac{3 c_{S} v_{L}}{r_{0} A^{1 / 3} T}\right)=\frac{T\left(A_{t o t}-A\right)}{V-A v_{L}},
$$

where $A$ is the mass number of the droplet and $A_{\text {tot }}$ is the total mass of the system (droplet plus vapour) and a strict mass conservation has been implemented. If we introduce the vapour fraction $x=1-A / A_{\text {tot }}$ and the reduced temperature $\tau=T / \Delta h_{0}$, the relation between $\tau$ and $x$ (which is monotonically correlated to the energy) at constant volume can be written as

$$
c_{1}-\frac{1}{\tau}+\frac{c_{2}}{\tau(1-x) A_{t o t}^{1 / 3}}=\log \tau+\log \frac{x}{1-c_{3}(1-x)} .
$$

Here $c_{1}=\log \left(p_{0} / \rho \Delta h_{0}\right), c_{2}=3 c_{s} v_{L} / r_{0} \Delta h_{0}, c_{3}=\rho v_{L}$ contain the specific features of the physical system under study and are linked to its bulk pressure, surface properties and to the system volume respectively. In the thermodynamic limit $A_{\text {tot }} \rightarrow \infty$ the relation between the temperature and the vapour fraction (i.e. the caloric curve) equation (37) is monotonic in the physical domain $0<x<1$ for all physical values of the constants as expected. For finite systems the equation of state can present a backbending with an amplitude depending on the value of the parameters $c_{1}, c_{2}, c_{3}$. An example is given in Figure 18 for $c_{1}=1, c_{2}=0.25$, $c_{3}=0.1$. The quantity $c_{1}$ represents a global shift and does not influence the monotonic character of the equation of state. The quantity $c_{2}$ governs the speed of convergence towards the thermodynamic limit while the influence of $c_{3}$ is shown in the right part of Figure 18. The backbending progressively decreases with the 


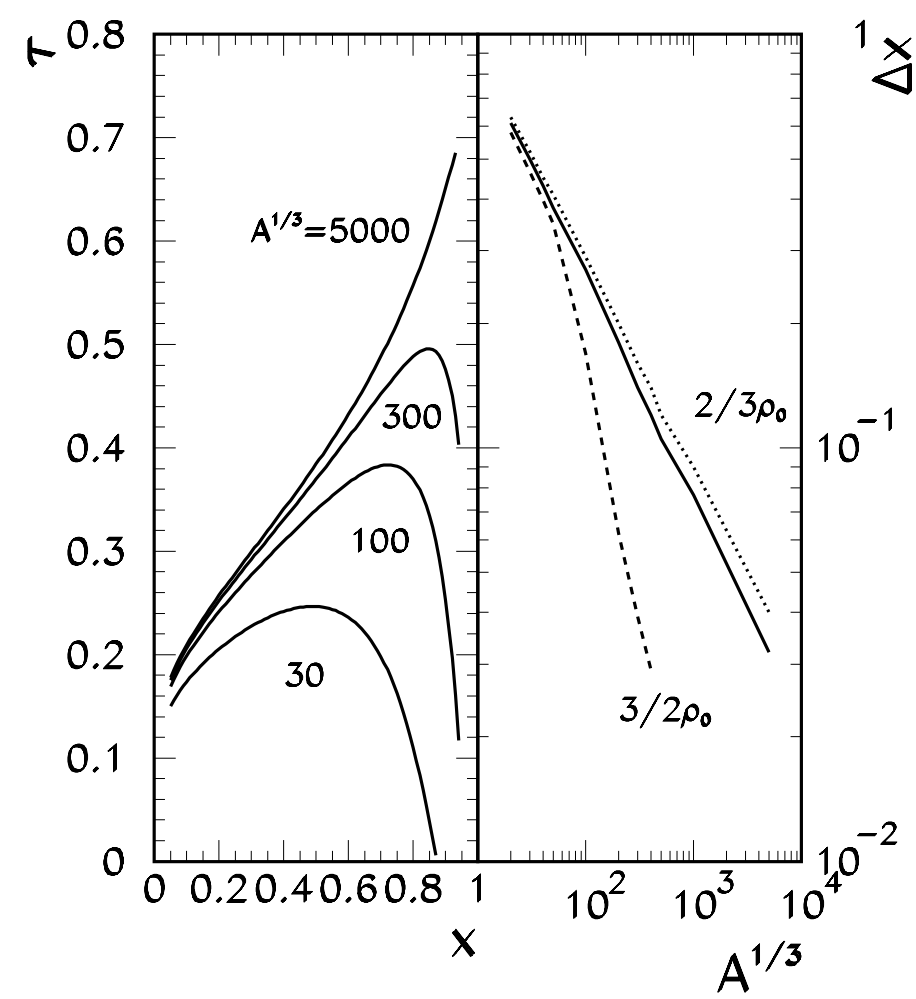

Figure 18. Equation of state of a liquid droplet in equilibrium with its vapour from equation (37). Left side: reduced temperature as a function of the vapour fraction for different sizes of the system. Right side: vapour fraction interval of the negative heat capacity region as a function of the size of the system. Full line: same total density as in the calculation of the left panel; dashed (dotted) line: higher (lower) density.

increasing size of the system, but this phenomenon is not specific of very small droplets only. In order to quantify the evolution towards the thermodynamic limit one may study the variation of the extension of the backbending region with the size of the system. To this aim, we have represented the vapour fraction interval corresponding to the slope inversion of the equation of state in the right part of Figure 18. The monotonous correlation between the energy and the vapour fraction assures that the backbending in $x$ corresponds to a backbending in energy, i.e. the adimensional quantity $\Delta x$ is directly correlated to the energy jump in the backbending region. The clear power law behaviour as a function of the total mass of the system shows that the energy jump goes to zero only at the thermodynamic limit.

This schematic example shows that, even in standard first order phase transitions (here we have a volume jump at the transition pressure in the thermodynamic limit, see Eq. (36)) we can observe transient backbending behaviours (here 
the caloric curve Fig. 18) that converge to a monotonous equation of state [19]. More important, the convexity anomalies associated to first order phase transitions can be relevant even on a mesoscopic scale. An interesting consequence of that is that the value of physical observables can be drastically different in the different ensembles still at a mesoscopic scale. As an example, a caloric curve is always by definition monotonic in the canonical ensembles while we have seen that temperature can decrease for increasing excitation if the system is isolated.

Of course the quantity called temperature is not defined in the same way in the two ensembles; as long as ensembles are not equivalent and $\beta \neq \partial_{E} \ln W$ we can wonder whether $\partial_{E} \ln W$ still represents the physical temperature of the system: does the anomaly that we have analytically recognized in the density of states really imply that pumping energy out of a system heats it up, or is it rather a mathematical curiosity? This question, addressed in the next section, can of course be generalized to any intensive variable conjugated to an order parameter in a generic first order phase transition.

\subsection{What is temperature? (I)}

We all know that the second law of thermodynamics states that temperature measures the increase rate of entropy, $d S=\delta Q / T$ where $Q$ is the (disordered) thermal energy we have called $E$ in these lectures. Let us show that the rate of entropy is indeed the response of a thermometer loosely coupled to the system under study. In such a case, loosely coupled means that the states of the total system are the independent tensorial products of the states of the thermometer times the one of the system. Moreover, the total energy is also simply the sum of the two partial energies.

From a macroscopic point of view, the equilibrium between the system and the thermometer requires the total entropy $S_{\text {tot }}=S_{s y s}+S_{\text {therm }}$ to be a maximum under the constraint of the total energy $E_{\text {tot }}=E_{s y s}+E_{\text {therm }}$. The definition $T^{-1}=d S / d E$ leads then to the equality of temperatures for the system and the thermometer, $T_{\text {sys }}=T_{\text {therm }}$ in agreement with the zeroth's law of thermodynamics.

However we have also seen in the first chapter that starting from the same Shannon information kernel many different entropies exist, according to the different constraints that define the equilibrium under study. The various entropies only converge toward a unique quantity at the thermodynamic limit, if this latter exists. On the other side the quantity that backbends is only the microcanonical temperature $T^{-1}=d \ln W / d E$. We therefore ask the question whether a physical thermometer applied to an isolated system measures the microcanonical temperature, i.e. whether the negative heat capacity discussed above is a real measurable physical phenomenon. A thermometer by definition loosely interacts with the system. This means that if a thermometer (of energy $E_{\text {therm }}$ ) is put into a system (of energy $\left.E_{s y s}\right), E_{\text {therm }}$ and $E_{s y s}$ can be considered as independent variables. The ensemble of system plus thermometer is isolated with a total energy $E_{\text {tot }}$, therefore the equiprobability of microstates and the factorization of the Hilbert space into the system and thermometer parts, leads to the equilibrium probability 
distribution for the thermometer energy

$$
p_{E_{t o t}}\left(E_{t h}\right)=\frac{W_{t h}\left(E_{t h}\right) W_{s y s}\left(E_{t o t}-E_{t h}\right)}{W_{t o t}\left(E_{t o t}\right)},
$$

where $W_{t h}\left(W_{\text {sys }}\right)$ is the number of states of the thermometer (system) and where $W_{t o t}\left(E_{t o t}\right)=\int d E_{t h} W_{t h}\left(E_{t h}\right) W_{s y s}\left(E_{t o t}-E_{t h}\right)$ is the number of states of the total system (thermometer + system). Temperature is then defined as the response of the thermometer in the most probable state; if we maximize the probability (38) we get

$$
\frac{d \ln W_{t h}}{d E_{t h}}=\frac{d \ln W_{s y s}}{d E_{s y s}},
$$

which shows that the quantity shared at the most probable energy partition, is indeed the microcanonical temperature.

This result is not in contradiction with the standard idea that for a thermostat, the physical temperature is the intensive variable conjugated to the energy, i.e. the (inverse of the) $\beta$ Lagrange multiplier. Indeed if we consider a thermometer inside a thermal bath, its most probable energetic state will be given by the maximum of the canonical distribution of energies

$$
p\left(E_{t h}\right)=W_{t h}\left(E_{t h}\right) \exp \left(-\beta E_{t h}\right) / Z_{\beta},
$$

which gives an energy $\bar{E}_{t h}$ such that $\partial_{E} \ln W_{t h}\left(\bar{E}_{t h}\right)=\beta$.

Equation (38) shows that from a microscopic point of view the temperature is indeed an ensemble property: the properties of a thermometer fluctuate from event to event. Only at the thermodynamic limit (for both the system and the thermometer) the fluctuations will be reduced to zero, and the distribution of thermometer responses to a unique value.

An example of thermometer is given by the kinetic energy: indeed for a classical system (Eq. (38)) holds with $E_{s y s}=E_{\text {pot }}$ and $E_{t h}=E_{k i n}$. Equation (39) then implies that the most probable kinetic energy measures the microcanonical temperature.

\section{A "typical" finite system: the explosion of a supernova}

In the previous chapter we have shown that negative heat capacities systematically occur in first order phase transitions of finite systems, when energy is an order parameter. From an historical point of view however, the problematics linked to negative heat capacity has started in the early seventies in a very different context, namely as a specificity of self-gravitating systems. Indeed a star that has exhausted its nuclear fuel radiates (i.e. loses energy) and heats up because of the gravitational contraction, i.e. behaves as a negative heat capacity system. Such a thermodynamic interpretation of the last steps of stellar evolution was considered 
as a triviality by the astronomers and as a absurdity by thermodynamicists. Let us summarize the two opposite arguments here.

- The astronomers' point of view. If we consider an isolated system with a potential energy $\propto r^{-n}$, the virial theorem states

$$
2 E_{\text {kin }}+n E_{\text {pot }}=0 .
$$

In the case of the gravitational potential $n=1$ and we get for $N$ particles $E_{\text {tot }}=-E_{\text {kin }}=-3 / 2 N T$ which implies $C=-3 / 2 N<0$, showing that a self-gravitating object has always $C<0$.

- The thermodynamicists' point of view. For a generic equilibrated system in the canonical ensemble

$$
C=-\beta^{2} \frac{d\langle E\rangle}{d \beta}=\beta^{2}\left\langle(E-\langle E\rangle)^{2}\right\rangle>0,
$$

showing that the heat capacity corresponds to the energy fluctuation which cannot be negative.

In the following sections we will show that both statements are incorrect, and that negative heat capacity in macroscopic self-gravitating systems is a physical equilibrium phenomenon exactly equivalent to the first order phase transitions in finite systems studied in the last chapter.

Indeed a mesoscopic boiling droplet and an collapsing (or exploding) star are very similar in the sense that in both cases the size of the system is comparable to the range of the force.

\section{1. $\quad \mathrm{C}<0$ in self-gravitating systems and the gravothermal catastrophe}

The confusion arising from the two contradictory arguments about the sign of the heat capacity was increased by the famous gravothermal catastrophe predicted first by Antonov [20] from the study of the extrema of the microcanonical entropy of a self-gravitating object in the mean field approximation as a function of the one body matter density. The one body Shannon entropy reads

$$
S=-\int d^{3} r d^{3} p f(\vec{r}, \vec{p}) \ln f(\vec{r}, \vec{p}) .
$$


The microcanonical equilibrium can be found as usual, by maximizing the entropy in a fixed volume $V$ under the particle number and energy constraint (see Sect. 1, Chap. 1)

$$
\begin{aligned}
& 0=d S-\alpha d N-\beta d E \\
& N=\int d^{3} r d^{3} p f(\vec{r}, \vec{p}) \\
& E=\int d^{3} r d^{3} p \frac{p^{2}}{2 m} f(\vec{r}, \vec{p})-\frac{G m^{2}}{2} \int d^{3} r d^{3} p d^{3} r^{\prime} d^{3} p^{\prime} \frac{f f^{\prime}}{\left|\vec{r}-\vec{r}^{\prime}\right|} .
\end{aligned}
$$

The result is a self-consistent equation for the equilibrium one body matter density $\rho(\vec{r})=\int d^{3} p f(\vec{r}, \vec{p})$ that can be solved to give the density contrast $\rho_{c} / \rho_{e}$ between the center and the surface of the box. The result is that the density contrast is an increasing function of the volume. Figure 19 shows an exact calculation of this problem by Lynden-Bell for the case of identical hard spheres [21]. For extremely large volumes $\rho_{c} / \rho_{e}>709$ the extremum of the entropy is a minimum meaning that no equilibrium solution exists in this case (Antonov gravothermal catastrophe). This result looks particularly strange since the instability provoked by the gravitational potential does not appear when the box is too small (and the interaction energy is strong) but when the box is too large (and the interaction energy is loose). Moreover for intermediate boxes $32.2<\rho_{c} / \rho_{e}<709$ the extremum is a maximum, i.e. the solution is stable, but the heat capacity contains a pole and a negative branch, which traditionally was associated to an instability (see Sect. 3, Chap. 1).

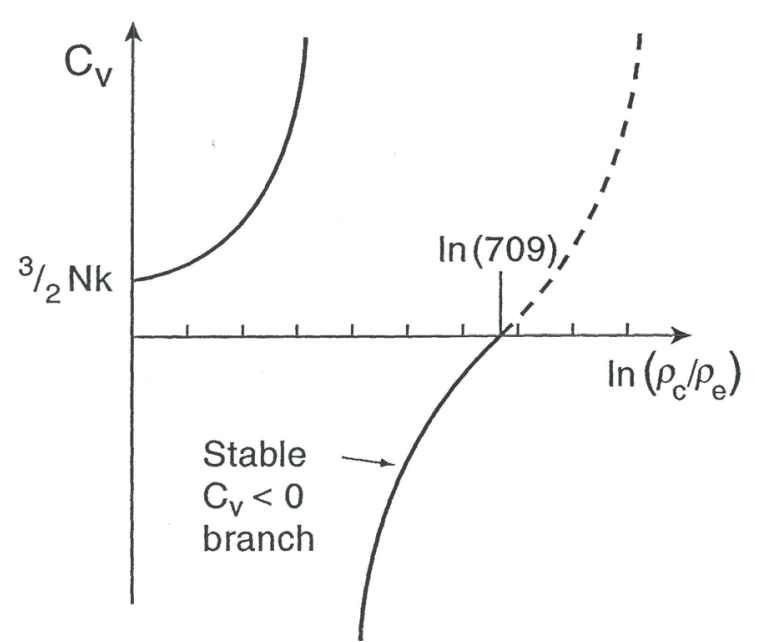

Figure 19. Microcanonical heat capacity as a function of the density contrast for hard spheres with gravitational interactions. Reprinted by permission from Blackwell Publishing Ltd [D. Lynden-Bell, Monthly Not. Roy. Astr. Soc. 138, 495 (1968)], copyright 2005. 


\subsection{Solution of the Antonov paradox}

The disagreement between the gravothermal catastrophe and equation (41) clearly implies that the Van Hove theorem (see Sect. 4.1, Chap. 1) is violated. This can only be due to the long range of the force. The incoherence with the virial theorem (Eq. (40)) can be explained by considering that if we are dealing with $N$ particles the density of states (see Sect. 4.3, Chap. 1)

$$
W \propto \int d^{3 N} r\left(E-\sum_{i j} \frac{m_{i} m_{j}}{r_{i j}}\right)^{3 N / 2}
$$

diverges unless a short range cut-off and a constraining potential (or boundary condition) are introduced. This means that the virial theorem has to be corrected for the short range repulsion and for the boundary condition. A schematic modelization of this problem has been proposed by Thirring as a constant interaction among all particles inside a volume $V_{0}<V$ and no interaction outside [22]. This model is equivalent to the two coupled harmonic oscillator problem proposed in Section 4.3 (Chap. 1). The finite liquid drop of Section 4.3 (Chap. 1) is now an infinite star, while the saturated vapour corresponds to the star atmosphere. As for the model (Sect. 4.3, Chap. 1), for the two boxes model a $C<0$ is found [22] whenever the difference between the two volumes is important, i.e. for big total volumes, in agreement with the Lynden-Bell result [21]. Following the reasoning of Section 4 (Chap. 1), we can then associate the $C<0$ regime to a phase coexistence between the star and its atmosphere that stabilizes the stellar nuclear plant, and the gravothermal catastrophe to a first order phase transition when the nuclear fuel is exhausted.

If we consider the generic case for the interaction in the dense phase $E=\varepsilon N^{\gamma}$ we can study the effect of the range of the force on the heat capacity in the thermodynamic limit. The resulting caloric curves are given in Figure 20 [22]. In agreement with Section 4 (Chap. 1), we can see that the negative heat capacity disappears at the thermodynamic limit for short range interactions $(\gamma=1)$ but all ranges $\gamma>1$ lead to a backbending that is preserved when $N \rightarrow \infty$, i.e. this phenomenon is not specific of the gravitational interaction. We can also understand why the same phenomenology appears in self-gravitating macroscopic systems and in small short ranged systems, as long as the range of the force is comparable with the linear dimension of the system.

\subsection{Thermal contact between $\mathbf{C}<0$ systems}

Phase coexistence in standard macroscopic thermodynamics is a trivial phenomenon: the tangent construction implies that the thermodynamic properties of phase coexistence are completely determined by the properties of the corresponding pure phases. On the other hand if surfaces cannot be neglected respect to bulk properties (i.e. if the system is finite or the interaction is long range), new unexpected phenomena peculiar to the coexistence phase appear, as negative 


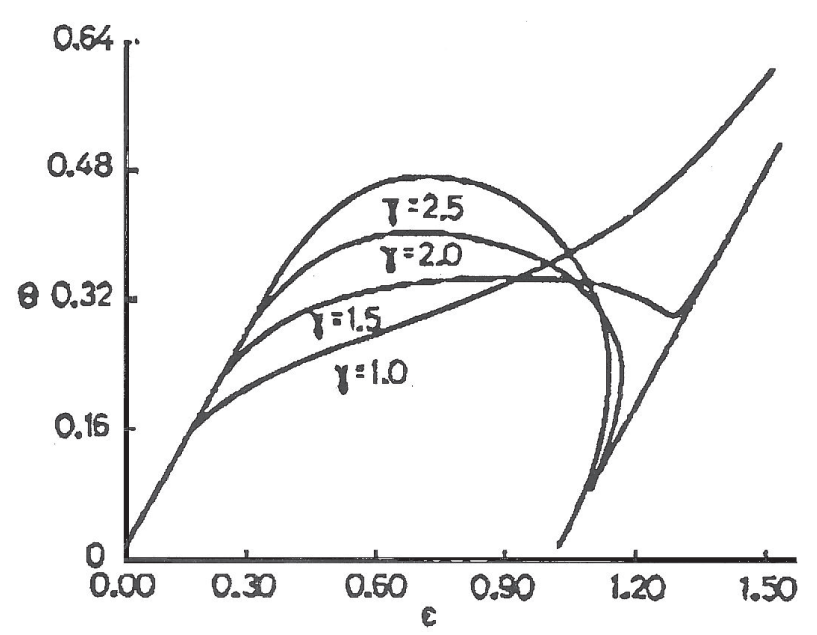

Figure 20. Caloric curves in the subcritical regime for different ranges of the interaction. From W. Thirring [Z. Phys. 235, 339 (1970)], reprinted with kind permission of Springer Science and Business Media.

compressibility or negative heat capacity. The peculiar thermodynamic properties of the coexistence phase can be better understood if we consider in some detail the problem of thermal contact between $C<0$ systems.

Let us consider a system with $C_{1}<0$ in thermal contact with a second system with a heat capacity $C_{2}$. Let us distinguish some different cases.

1. If $C_{2}<0$ no equilibrium is possible between the two systems. Indeed if $T_{2}>T_{1}$ energy will be transferred from system (2) to system (1) and system (2) will get even hotter while (1) becomes even cooler. This implies that it is not possible to divide a $C<0$ system into two parts each with $C<0$; in other words $C<0$ systems are not extensive.

2. A thermal equilibrium is possible if the $C_{1}<0$ system is in contact with a $C_{2}>0$ system small enough such that $C_{2}<\left|C_{1}\right|$. In this case the temperature increase of the initially slightly hotter system (1) is compensated by system (2) which has positive heat capacity and receives energy, and therefore increases its temperature more rapidly; a final equilibrium state will then be achieved with $T_{e q}>\max \left(T_{1}, T_{2}\right)$. In particular if the two systems are independent, we can write for the microcanonical distribution of energy $E_{1}$ : $\ln P_{E}\left(E_{1}\right)=S_{1}\left(E_{1}\right)+S_{2}\left(E_{2}\right)-S_{t o t}(E)$ where $E=E_{1}+E_{2}$. The stability condition (i.e. the curvature of $\ln P$ around the extrema) can then be written as

$$
\frac{d^{2} S_{t o t}}{d E_{1}^{2}}=-\frac{1}{2 T^{2}}\left(\frac{1}{C_{1}}+\frac{1}{C_{2}}\right)<0,
$$

where $T_{1}=T_{2}=T$ is the equilibrium temperature (the microcanonical temperature associated with the most probable energy partition). This is 
the case of a living star: the central part has $C_{1}<0$, the surface has $C_{2}>0$, the global system (or coexistence phase) has $C=C_{1}+C_{2}<0$ and is stable, since the core transfers energy to the atmosphere in the form of radiation. The gravothermal catastrophe occurs when $C=0$ (see Fig. 19), when the gravitational contraction cannot be stopped by nuclear reactions leading to the whole well-known phenomenology of stellar collapse [23,24].

3. A C $<0$ system cannot reach thermal equilibrium with a thermostat. Indeed if (1) is much smaller than (2) (Eq. (42)) is violated and the total entropy is a minimum. This implies that a negative heat capacity is impossible in the canonical ensemble coherently with equation (41): the $C<0$ system in contact with a thermal bath stops to hesitate between the two coexisting phases and jumps to its stable equilibrium state, i.e. makes a phase transition. These considerations allow to gain an intuitive understanding of the fact that the $C<0$ of a boiling liquid drop (see Sect. 4, Chap. 1) transforms into a plateau when a water kettle is put on a gas cooker in the macroscopic world. Indeed any microscopic portion of the boiling portion once isolated would appear as a microcanonical $C<0$ system; the thermal contact with the rest of the system (which can be considered a thermostat because of the short range of the interaction) forces the otherwise stable $C<0$ system to choose between the liquid and the gas solution, giving rise to the Maxwell construction.

All we have discussed in this chapter has been presented in the early seventies; it may be surprising then that negative heat capacity has not been recognized at this time as a general paradigm of phase coexistence in non-extensive (finite or infinite) systems. Indeed it is only in the recent years, following the success of the experimental application of these ideas to mesoscopic systems (see the second part of this work) that these concepts have been developed further. The conceptual difficulty of accepting a stable equilibrium with negative heat capacity is due to different reasons.

First, the inhomogeneity of the star makes it difficult to consider it as a single thermodynamic object; following the reasoning of Section 4 (Chap. 1) we however understand that on the contrary this inhomogeneity is an essential feature of the thermodynamics of coexisting phases.

Moreover the fact that the energy exchange between the star and the atmosphere consists in radiation (taking their energy from nuclear reactions) makes the problem an out of equilibrium transport problem.

Most important, the fact that it is not possible to define a canonical equilibrium for a $C<0$ system means that the validity itself of such an equilibrium is not easy to understand: how can one speak of temperature in the absence of thermal contact, and what is in this case the meaning of a temperature decrease with increasing energy? We have already discussed the problem of the physical meaning of the microcanonical temperature in Section 4.7 (Chap. 1); let us just recall here that if a $C<0$ cannot be put in contact with a thermostat, on the other hand a thermometer put in a $C<0$ system is perfectly well defined, and measures the microcanonical temperature. This is the case 2 of the discussion above (with $C_{1}<0$ and $C_{2}<\left|C_{1}\right|$ ). 


\section{Abnormal topology of event distributions}

In most textbooks the equivalence between the different statistical ensembles is either postulated or demonstrated at the thermodynamic limit through the Van Hove theorem (see Sect. 4.1, Chap. 1).

In the previous chapters we have shown that ensembles may not be equivalent. For finite systems, two ensembles which put different constraints on the fluctuations of the order parameter lead to very different equations of states close to a first order phase transition. As an example, the microcanonical heat capacity may diverge to become negative while the canonical one remains always positive and finite (see Sect. 4.2, Chap. 1). In Section 5 we have moreover seen that such inequivalences may survive at the thermodynamic limit for systems involving long range forces.

In this chapter we will try to formalize all these findings looking at the general properties of the order parameter distribution. This will allow us to propose a definition of phase transitions in finite systems also for intensive ensembles (i.e. ensembles where the order parameter is not controlled on an event by event basis) based on topology anomalies of the event distribution in the space of observations. We will show in the next sections that this generalizes the definitions based on the curvature anomalies of thermostatistical potentials presented in Section 4 (Chap. 1). Such a definition gives a clear understanding of the physical meaning of an order parameter as the best variable to separate the two maxima of the distribution, and can be directly used experimentally (see Sect. 4.1, Chap. 2).

In Sections 6.7, 6.8 and 6.9 (Chap. 1), we will come back to the problem of the thermodynamic limit. If the order parameter is sufficiently collective, the topologic anomaly represented by the state change of the finite system may survive until the infinite volume and infinite number limit. Looking at the distribution of zeroes of the partition sum in the complex Lagrange parameter plane we will demonstrate in Section 6.8 that this requirement is equivalent to the Yang Lee unit circle theorem [25] that connects the finite size state density anomaly to the bulk phase transition. Finally in Section 6.9 we will briefly analyze the conceptual consequences of an ensemble inequivalence preserved up to the thermodynamic limit as in the case of the gravitational interaction: based again on the topologic properties of the order parameter distribution, a sufficient condition will be worked out for the failure of standard macroscopic thermodynamic approaches to first order phase transitions.

\subsection{Negative heat capacity and bimodal energy distributions}

Let us first concentrate on finite systems. To begin we will consider the specific example of the microcanonical and the canonical ensemble, characterized by the energy $E$ and the temperature $\beta^{-1}$ respectively. 
In Section 4.7 (Chap. 1) we have shown that the extrema of the probability distribution in the canonical ensemble

$$
P_{\beta}(E)=\exp \left(S(E)-\beta E-\log Z_{\beta}\right)
$$

are given by the equation

$$
T^{-1} \equiv \partial_{E} S\left(\bar{E}_{\beta}\right)=\beta
$$

If this extremum is unique, we can perform a saddle point approximation around the most probable energy $\bar{E}_{\beta}$ leading to the average energy

$$
\langle E\rangle_{\beta}=\int d E E e^{-\frac{\left(E-\bar{E}_{\beta}\right)^{2}}{2 C}} g_{\beta}(E-\bar{E}),
$$

with $g_{\beta}(x)=c_{0}+c_{3} x^{3}+c_{4} x^{4}+\ldots$ If $P_{\beta}$ is symmetric, $\langle E\rangle_{\beta}=\bar{E}_{\beta}$ and $T^{-1}(\langle E\rangle)=\beta$ meaning that the microcanonical caloric curve $T(\bar{E})$ exactly coincides with the canonical one $\beta^{-1}(\langle E\rangle)$. If the distribution is not symmetric some corrective terms arise: $\langle E\rangle_{\beta}=\bar{E}_{\beta}+\delta_{\beta}$, where $\delta_{\beta}=\int d x x \exp \left(-x^{2} / 2 C\right) \tilde{g}_{\beta}(x)=3 c_{3} \sqrt{2 \pi C^{5}}+\ldots$ with $\tilde{g}_{\beta}$ the series of the odd terms of $g_{\beta}$. However, the shift $\delta$ is in most cases small, so that when $P_{\beta}$ has a unique maximum the ensembles are almost equivalent even for a finite system. The same approximation can be applied to the partition sum, which is linked to the entropy by an exact Laplace transform

$$
Z_{\beta}=\int d E W(E) e^{-\beta E}
$$

leading to

$$
Z_{\beta} \approx W(\bar{E}) e^{-\beta \bar{E}} \approx W(\langle E\rangle) e^{-\beta\langle E\rangle},
$$

which corresponds to a simple linear transformation between the thermodynamic potentials, i.e. an approximate Legendre transform

$$
\ln Z_{\beta} \approx \ln W(\langle E\rangle)-\beta\langle E\rangle
$$

As we have already mentioned in Section 2.1 (Chap. 1), this expression has not to be mixed up with the true (and exact) Legendre transform $\ln Z_{\beta}=S_{\beta}(\langle E\rangle)-\beta\langle E\rangle$ which gives the relation between the partition sum and the Shannon entropy within the canonical ensemble.

However in first order phase transitions $P_{\beta}$ has a characteristic bimodal shape (see Sect. 2.2,Chap. 1) [26-28] with two maxima $\bar{E}_{\beta}^{(1)}, \bar{E}_{\beta}^{(2)}$ that can be associated with the two phases and a minimum $\bar{E}^{(0)}$. These three solutions of equation (43) imply a backbending for the microcanonical caloric curve. Indeed a minimum of $P_{\beta}$ corresponds to a convexity of the entropy according to $d_{E}^{2} \ln P_{\beta}=d_{E}^{2} \ln W$. A single saddle point approximation is not valid in this case; however it is always possible to write $P_{\beta}=m_{\beta}^{(1)} P_{\beta}^{(1)}+m_{\beta}^{(2)} P_{\beta}^{(2)}$ with $P_{\beta}^{(i)}$ mono-modal normalized probability 
distribution peaked at $\bar{E}_{\beta}^{(i)}$. The canonical mean energy is then the weighted average of the two energies

$$
\langle E\rangle_{\beta}=\tilde{m}_{\beta}^{(1)} \bar{E}_{\beta}^{(1)}+\tilde{m}_{\beta}^{(2)} \bar{E}_{\beta}^{(2)},
$$

with $\tilde{m}_{\beta}^{(i)}=m_{\beta}^{(i)} \int d E P_{\beta}^{(i)}(E) E / \bar{E}_{\beta}^{(i)} \simeq m_{\beta}^{(i)}$, the last equality holding for symmetric distributions $P_{\beta}^{(i)}$. Since only one mean energy is associated with a given temperature $\beta^{-1}$, the canonical caloric curve is monotonous. In particular in the backbending region the mean energy is an interpolation between the two energies $\bar{E}_{\beta}^{(1)}$, $\bar{E}_{\beta}^{(2)}$ associated with the considered $\beta$, the weighting factor being the probability of each phase $\tilde{m}_{\beta}^{(i)}$.

If instead of looking at the average $\langle E\rangle_{\beta}$ we look at the most probable energy $\bar{E}_{\beta}$, this (unusual) canonical caloric curve is almost identical to the microcanonical one (see Eq. (43)) up to the transition temperature $\beta_{t}^{-1}$ for which the two components of $P_{\beta}(E)$ have the same height. At this point the most probable energy jumps from the low to the high energy branch of the microcanonical caloric curve. The most probable canonical energy is still a monotonic curve, but it presents a plateau at $\beta_{t}^{-1}$. Therefore the difference between the canonical and microcanonical caloric curves remains when one is looking at the most probable energy instead of the average.

The connection between the canonical energy distribution and the microcanonical equation of state is presented for the three dimensional lattice gas model in Figure 21. The bimodality of the canonical energy distribution as well as the discontinuity in the most probable energy value are definitions of the phase transition exactly equivalent to the convexity anomaly of the entropy discussed in Section 4 (Chap. 1) and clearly defined even for very small system (216 particles are considered for the calculation of Fig. 21).

\subsection{Definition of the transition point}

In the previous section we have seen that the transition temperature for the finite system can be unambiguously recognized from the energy jump of the most probable canonical energy as a function of temperature. In Sections 6.7 and 6.8 (Chap. 1), we will show that this definition has the correct asymptotic limit and is a necessary and sufficient condition for the occurrence of a first order phase transition in the bulk.

It is also easy to show that the energy jump at $\beta_{t}$ is equivalent to a tangent construction for the microcanonical caloric curve. Indeed defining $\beta_{t}$ as the inverse temperature at which the two maxima have the same height, $\ln P_{\beta}\left(\bar{E}_{\beta}^{(1)}\right)=$ $\ln P_{\beta}\left(\bar{E}_{\beta}^{(2)}\right)$, this implies for the entropy difference between the two phases

$$
S\left(\bar{E}_{\beta}^{(2)}\right)-S\left(\bar{E}_{\beta}^{(1)}\right)=\beta\left(\bar{E}_{\beta}^{(2)}-\bar{E}_{\beta}^{(1)}\right),
$$




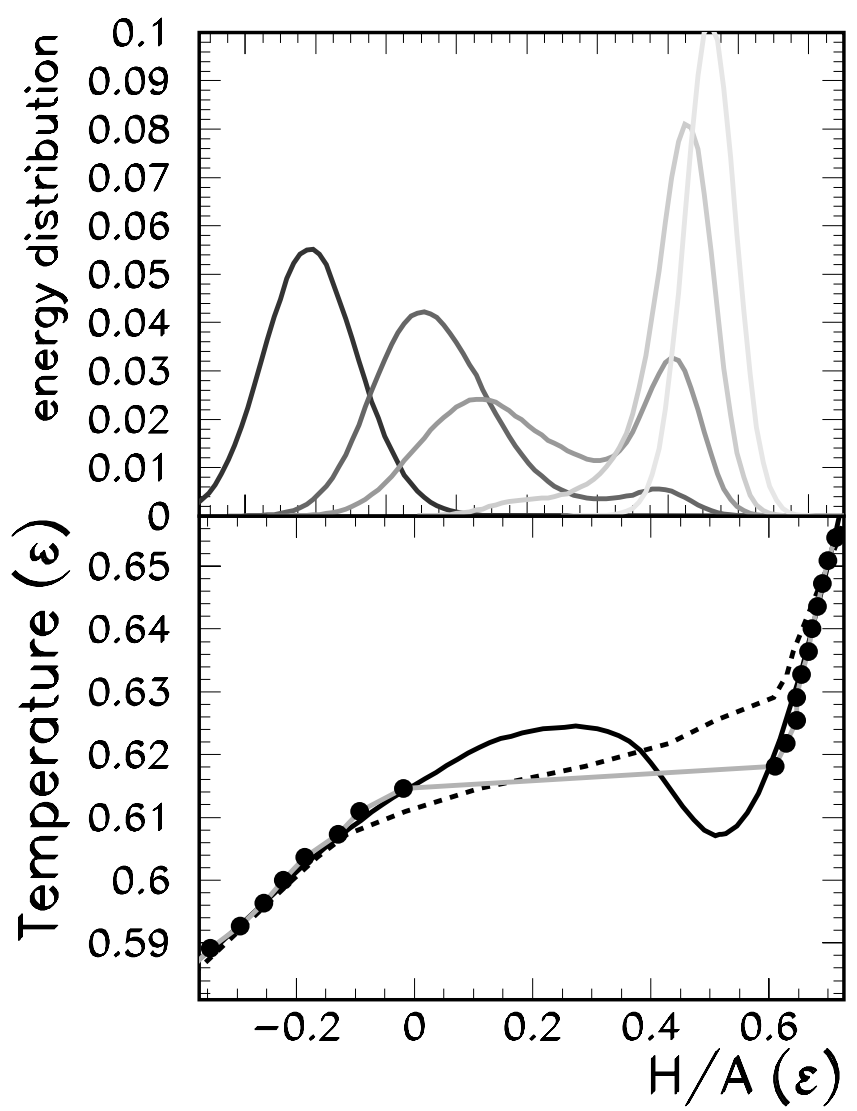

Figure 21. Canonical thermodynamics of 216 particles in the isobar lattice gas model. Upper part: energy distributions for different temperatures. Lower part: caloric curve from the average (dashed line) and the most probable (grey line and circles) energy. Full black line: microcanonical temperature.

which means for the microcanonical temperature

$$
\int_{\bar{E}_{\beta}^{(1)}}^{\bar{E}_{\beta}^{(2)}} \frac{d E}{T}=\beta\left(\bar{E}_{\beta}^{(2)}-\bar{E}_{\beta}^{(1)}\right) .
$$

This shows that, to be compatible with a tangent construction, at the transition temperature the two peaks must have the same height. In a finite system the widths are not negligible and a single phase is represented by the globality of each distribution and not only by its most probable value. Therefore at the transition temperature of a finite system the two phases do not necessarily have the same probability of occurrence, since this last property should require the equality of the surfaces rather than of the maxima. 


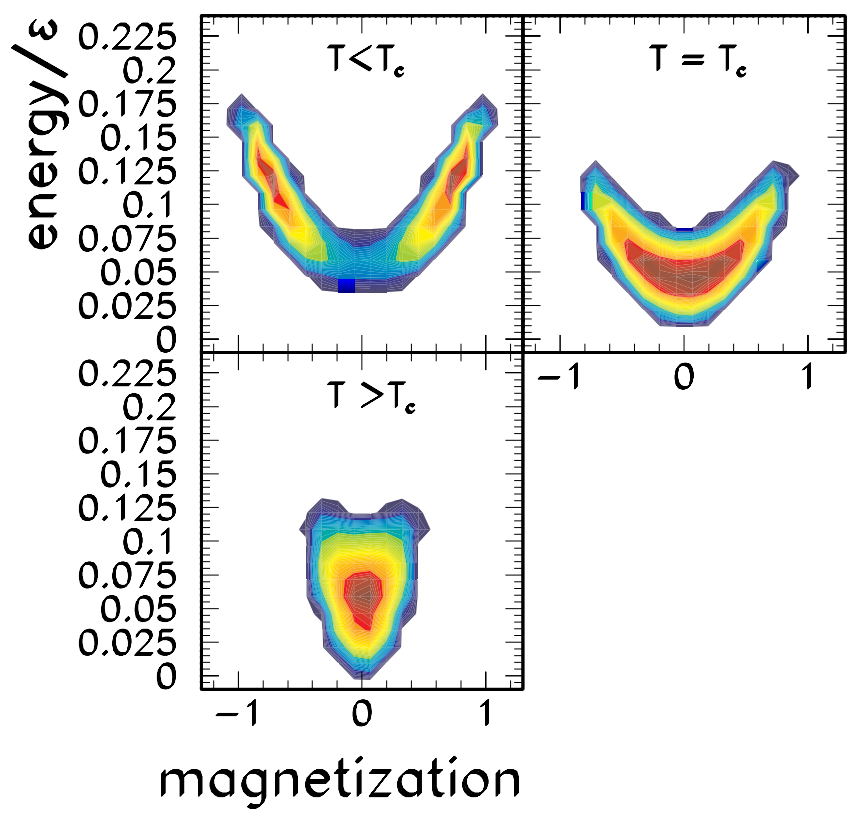

Figure 22. Event distribution in the energy versus magnetization plane for a $6 \times 6 \times 6$ Ising model with zero field at a subcritical, critical and supercritical temperature.

\subsection{Convexity anomalies and bimodal probability distributions}

This discussion can be generalized to any couple of extensive/intensive ensemble. Figure 22 shows the example of the Ising model at zero field. The bimodal structure in the $m$ direction corresponds to a negative susceptibility in a constant magnetization ensemble. In this case the projection on the energy axis does not show anomalies: the microcanonical heat capacity remains positive and the energy is not an order parameter. At $T_{c}$ the distribution presents a curvature anomaly only on the high energy side respect to the maximum. Therefore at this point the curvature passes through zero signalling a second order phase transition. Since in finite systems the canonical distribution for any $\beta, h(\mu)$ allows a complete exploration of the microcanonical entropy surface (in the limit of the total number of events analyzed), the whole microcanonical phase diagram can in principle be drawn from any single canonical temperature using the fact that the distribution is in fact the entropy

$$
S(E)=\log P_{\beta, h=0}(E, M)+\ln Z_{\beta, h=0}+\beta E .
$$

As an example, the "croissant" shape of the distribution at $T_{c}$ not only defines the critical energy $e_{c}$ and magnetization $m_{c}$ of the second order phase transition but also allows to infer the coexistence line where the first order phase transition 
takes place. Indeed a constant energy cut of the distribution above $e_{c}$ directly represents the entropy as a function of magnetization and has a bimodal shape.

\subsection{Liquid gas phase transition and volume fluctuations}

Let us now take the example of the liquid-gas phase transition in a system of $n$ particles for which the volume is not controlled on an event by event basis but is at best known in average. In such a case, we can define an observable $\hat{B}_{1}$ as a measure of the size of the system; for example the cubic radius $\hat{B}_{1}=(4 \pi / 3 n) \sum_{i} \hat{r}_{i}^{3} \equiv \hat{V}$ where the sum runs over all the particles. Then a Lagrange multiplier $\lambda_{V}$ has to be introduced which has the dimension of a pressure divided by a temperature, $p=\lambda_{V} T$. In a canonical ensemble with an inverse temperature $\beta$ we can define different distributions which are illustrated in Figure 23. A complete information is contained in the distribution $P_{\beta \lambda_{V}}(E, V)=\bar{W}(E, V) Z_{\beta \lambda_{V}}^{-1} \exp -\left(\beta E+\lambda_{V} V\right)$ since events are sorted according to the two thermodynamic variables, $E$ and $V$. This leads to the density of states $\bar{W}(E, V)$ with a volume $V$ and an energy $E$. One can see that in the first order phase transition region the probability distribution is bimodal. In the spirit of the principal component analysis we can look for an order parameter $\hat{Q}=x \hat{H}+y \hat{V}$ which provides the best separation of the two

\section{Probability (a.u.)}

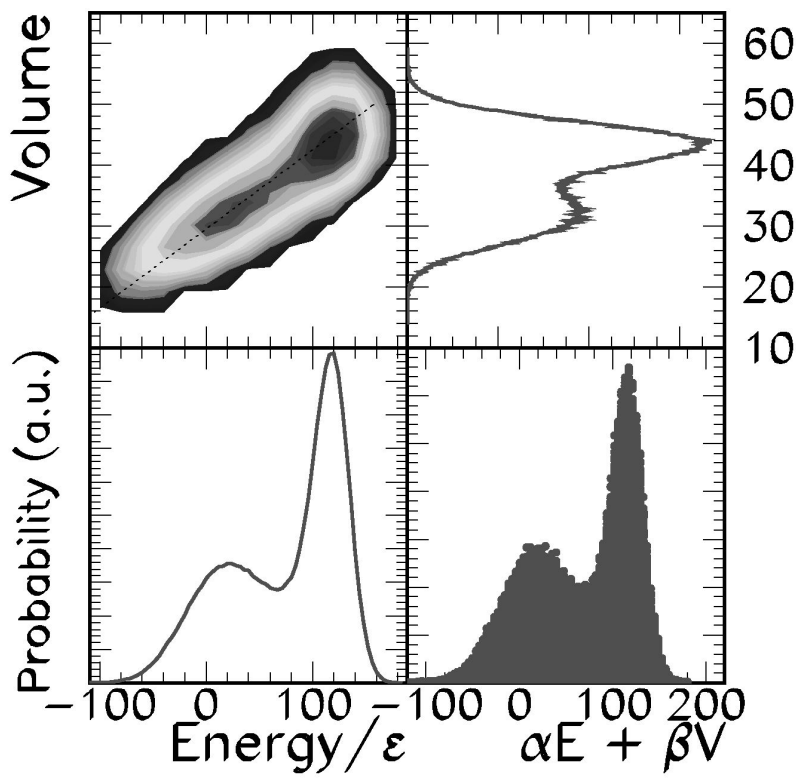

Figure 23. Event distribution in the volume versus energy plane for 216 particles in the isobar canonical lattice gas model and projection over the two axes. Bottom right: projection of the distribution over the direction separating at best the two distributions. 
phases. A projection of the event on this order parameter axis is also shown in Figure 23. Not surprisingly, the order parameter in this example is identified as the free enthalpy $H=E+p V$ (this is by the way the variable that has been used for the event sorting in Fig. 21 above). On the other hand if we cannot measure both the volume $V$ and the energy $E$, we are left either with

$$
P_{\beta \lambda_{V}}(E)=\bar{W}_{\lambda_{V}}(E) Z_{\beta \lambda_{V}}^{-1} \exp (-\beta E)
$$

giving access to the microcanonical partition sum $\bar{W}_{\lambda_{V}}(E)$ at constant $\lambda_{V}$; or with the probability

$$
P_{\beta \lambda_{V}}(V)=\bar{Z}_{\beta}(V) Z_{\beta \lambda_{V}}^{-1} \exp \left(-\lambda_{V} V\right),
$$

leading to the isochore canonical partition sum $\bar{Z}_{\beta}(V)$. Since both probability distribution $P_{\beta \lambda_{V}}(E)$ and $P_{\beta \lambda_{V}}(V)$ are bimodal, the associated partition sum have anomalous concavity intruders, i.e. negative heat capacity as well as negative compressibility.

As a general statement, we can define a first order phase transition for any number of particles as a bimodality in the probability distribution of an arbitrary observable; any observable that allows to separate the two maxima of the event distribution can then be considered as an order parameter.

\subsection{A mesoscopic example: negative magnetic susceptibility}

To better explore the connection between the distribution of the order parameter and the equation of state, let us consider the Ising model at fixed magnetization [19]. The partition sum can be written as

$$
Z_{\beta}(m)=\int d E W(E, m) \exp (-\beta E)
$$

where $m=\sum_{i}^{N} s_{i} / N$ is the magnetization per site. The equation of state $\widetilde{h}(m)=$ $-\beta^{-1} \partial_{m N} \ln Z_{\beta}(m)$ in the constant magnetization ensemble can be calculated from an iterative evaluation of the partition sum $Z_{\beta}(m)$ according to equation (26).

As shown in Figure 24, the phase transition is signalled by a backbending (full black line in the right part of Fig.24) that replaces the discontinuity in the equation of state $\langle m\rangle(h)$ of the standard Ising model with partition sum

$$
Z_{\beta h}=\int d m d E W(E, m) \exp (-\beta(E-m N h)) .
$$

Note that in the case of this specific model finite size correction are particularly small, and an almost perfect jump is observed already for a $3 D$ lattice size of linear dimension $L=6$. Indeed the average (full grey line) and the most probable (dashed line) Ising magnetization are almost indistinguishable on the right part of Figure 24 (see the discussion on finite size corrections in Sect. 6.1, Chap. 1). 


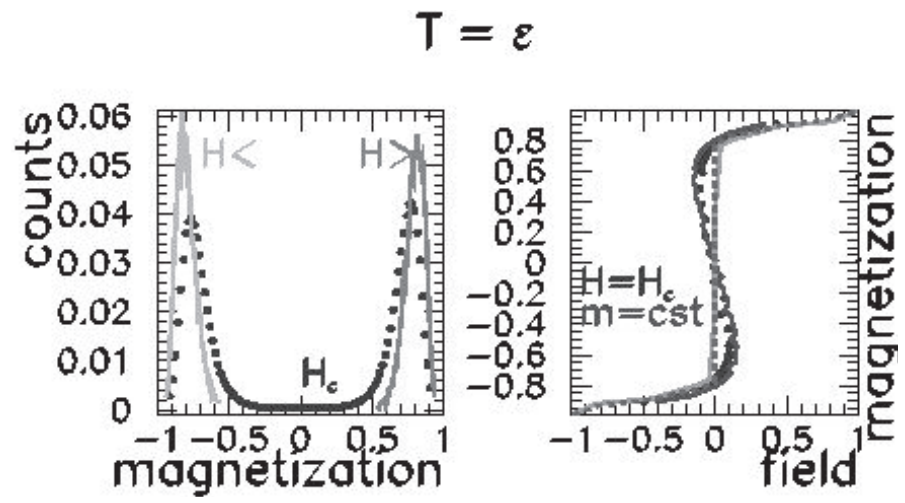

Figure 24. Left side: magnetization distribution in a $6 \times 6 \times 6$. Ising model at a subcritical temperature for three different values of the external field. Right side: equation of state for Ising and for the constant magnetization ensemble (backbending curve).

This same information concerning a negative magnetic susceptibility can be obtained by looking at the magnetization distribution of the standard Ising model with zero field

$$
P_{\beta h}(m)=\frac{1}{Z_{\beta h}} \int d E W(E, m) \exp (-\beta(E-m N h))=\frac{Z_{\beta}}{Z_{\beta h}} \exp (\beta m N h) .
$$

From equation (46) we can see that $\partial^{2} / \partial_{m}^{2} \ln P_{\beta h}=\partial^{2} / \partial_{m}^{2} \ln Z_{\beta}$. This shows that a minimum in the magnetization distribution (left part of Fig. 24) implies a convexity anomaly in the constant magnetization partition sum (symbols in the right part of Fig. 24) i.e. a negative magnetic susceptibility for mesoscopic ferromagnets sorted in constant magnetization bins.

Note also the very good agreement between the two independent calculations of $\partial / \partial_{m} \ln P_{\beta h}-\beta N h$ (symbols) and $\partial / \partial_{m} \ln Z_{\beta}$ (full line). In the first case the Ising sampling at zero field populates with negligible probability the microstates with $m=0$, while these are the only existing microstates for the calculation in the fixed magnetization ensemble, yet the two results coincide. This demonstrates the quality of our numerical sampling, and at the same time shows that the thermodynamics of an ensemble constrained by a conservation law can be inferred from the thermodynamics of the ensemble in which the conservation law is relaxed. In particular the observation of a bimodal probability distribution in an ensemble where the order parameter is not strictly fixed allows to conclude about the order of the phase transition in the corresponding extensive ensemble without explicitly observing or simulating it. This is true even in the transition region where the inequivalence between the ensembles does not concern only fluctuations, but is visible even at the level of mean values.

The connection between the bimodality of the magnetization distribution and a negative susceptibility is valid for all finite sizes up to the thermodynamic limit. Therefore, the well known fact that the Ising bimodality converges to a finite jump 
in the thermodynamic limit guarantees that the corresponding phase transition in the fixed magnetization Ising model is also first order up to the thermodynamic limit (see Sect. 4.1, Chap. 1).

Concerning the physical meaning of the equation of state in the Ising model with fixed magnetization, it is important to stress that $\widetilde{h}(m)$ is not a mathematical artifact but represents the physical magnetic field which, applied to standard Ising, gives $m$ as the most probable response. Indeed in the presence of an external field $h$, the most probable magnetization should fulfill

$$
\frac{\partial \log P_{\beta h}(m)}{\partial m}=\frac{\partial \log Z_{\beta}(m)}{\partial m}+\beta N h=\beta N(h-\widetilde{h}(m))=0,
$$

i.e. the (most probable) response is $m$ if the applied field is $\widetilde{h}(m)$. This is true as long as the system is not undergoing a phase transition i.e. as long as equation (47) has only one solution. In the transition region equation (47) has three solutions, two maxima and a minimum in between. This corresponds to the subcritical temperatures for which the Ising distribution $P_{\beta h}(m)$ is bimodal in a region around $h=0$. In this regime the interval $\Delta \widetilde{h}(m)$ associated with the backbending corresponds exactly to the interval $\Delta h$ for which $P_{\beta h}(m)$ is bimodal.

\subsection{Effect of the constraints on the order parameter}

Within this topologic definition of first order phase transitions, we have already observed that the order parameter is not unique, nor necessarily connected to a quantity conserved by the dynamics [13]: any observable $O$ which allows to separate the two phases, i.e. such that if events are sorted as a function of $O$, they split into two components separated by a minimum of the distribution function can be used as an order parameter (see Sect. 6.4, Chap. 1).

It is important to stress that this definition is valid only if (at least one of) the order parameters is free to fluctuate. If the order parameters are constrained by a conservation law, bimodalities have in general not to be expected. Let us consider for example the case of energy as an order parameter. In the microcanonical ensemble, the energy distribution being a $\delta$-function it cannot obviously be bimodal. Because of the loose correlation between the different observables in finite system where fluctuations are not negligible, we cannot a priori exclude that a bimodality may survive in some other observable loosely correlated with the energy. Indeed some models have been reported where the kinetic energy plays the role of an additional order parameter and shows a bimodal distribution in the microcanonical ensemble [29] (see Sect. 6.9). However in the general case the constraint of the conservation law on an order parameter reduces the variance of all observables correlated to it, such that the bimodalities are most often suppressed. In this case the first order character of the transition can only be recognized from the backbending of the equation of state relating the order parameter to its conjugated intensive variable as discussed in Section 4 of Chapter 1 (a backbending of the 


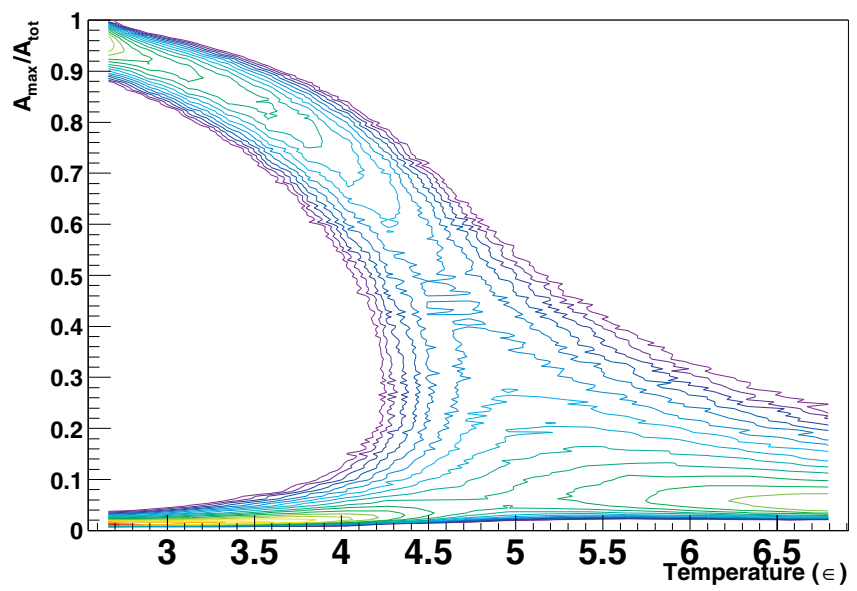

Figure 25. Distribution of the biggest fragment produced in every event as a function of the system temperature for a $6 \times 6 \times 6$ grancanonical lattice gas model at the critical chemical potential.

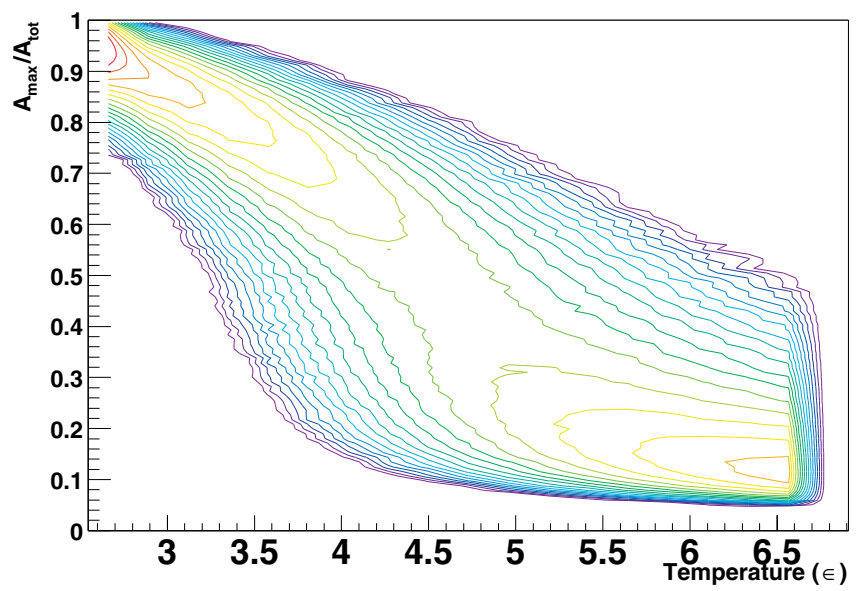

Figure 26. Distribution of the biggest fragment produced in every event as a function of the system temperature for a $6 \times 6 \times 6$ canonical lattice gas model at the critical density.

caloric curve in a microcanonical model, a negative susceptibility in a constant magnetization model, etc.).

An example is given in Figures 25 and 26 that show, in the standard isochore Lattice Gas model as a function of temperature, the behaviour of the size of the largest connected domain $A_{\max }$, here defined with the Coniglio-Klein algorithm [30] in its simplified version proposed by Campi and Krivine [31]. It is clear from Figure 25 that $A_{\max }$ is an order parameter: the event distribution splits into 
two separated components for all subcritical temperatures (here $T_{c} \approx 1.12 \epsilon \approx 6.5$ ). This is not surprising since with the particular cluster definition employed $A_{\max }$ is very well correlated to the total number of particles $A_{\text {tot }}$ which is the natural order parameter of the liquid gas phase transition in the isochore ensemble.

On the other hand because of this very same correlation, the implementation of a total mass conservation law in Figure 26 suppresses the bifurcation in the $A_{\text {max }}$ direction which characterizes the first order phase transition in the grancanonical ensemble. Still the correlation between $A_{\max }$ and $A_{\text {tot }}$ is sufficiently loose to allow to recognize the critical point from the maximum of the fluctuation and the jump in the mean value of $A_{\max }$.

These figures also illustrate the fact that a first order phase transition in a finite system can be seen as a bifurcation in the observable plane defined by the control variable (here the temperature) and (one of) the order parameter(s) [32], provided that the order parameter is not constrained by a conservation law in the considered sample.

\subsection{Topologic anomalies and the thermodynamic limit}

The definition of phase transition proposed in Section 6.3 can be applied to a wide range of situations even out of equilibrium [32], and can be directly implemented on experimental data (see Sect. 4.1, Chap. 2). However we have already stressed in Section 4.5 (Chap. 1) that not all topological anomalies will survive up to the bulk limit and give rise to a conventional thermodynamic phase transition. The transition between two isomeric states, the breaking of a Cooper pair, ionization, are all examples of state changes that do not lead to discontinuities in the bulk limit, i.e. do not converge to a thermodynamic first order phase transition. It is therefore of extreme interest to study the thermodynamic limit of the order parameter distributions. Since we have seen that the different statistical ensembles are in general not equivalent, the thermodynamic limit has to be considered separately for the intensive (say, canonical) and extensive (say, microcanonical) ensemble.

As we have already discussed in Section 4.1 (Chap. 1), the thermodynamic limit can be expressed as the fact that the thermodynamic potentials per particle converge when the number of particles $N$ goes to infinity: $f_{N, \beta}=\beta^{-1} \log Z_{\beta} / N \rightarrow \overline{f_{\beta}}$ and $s_{N}(e)=S(E) / N \rightarrow \bar{s}(e)$ where $e=E / N$. Let us also introduce the reduced probability $p_{N, \beta}(e)=\left(P_{\beta}(N, E)\right)^{1 / N}$ which then converges towards an asymptotic distribution $p_{N, \beta}(e) \rightarrow \bar{p}_{\beta}(e)$ where $\bar{p}_{\beta}(e)=\exp \left(\bar{s}(e)-\beta e+\bar{f}_{\beta}\right)$. Since $P_{\beta}(N, E) \approx$ $\left(\bar{p}_{\beta}(e)\right)^{N}$ one can see that when $\bar{p}_{\beta}(e)$ is normal the relative energy fluctuation in $P_{\beta}(N, E)$ is suppressed by a factor $1 / \sqrt{N}$. At the thermodynamic limit $P_{\beta}$ reduces to a $\delta$-function and the ensemble equivalence is recovered (see Sect. 2.2, Chap. 1).

To analyze the thermodynamic limit of a bimodal distribution $p_{N, \beta}(e)$, let us introduce $\beta_{N, t^{\prime}}^{-1}$, the temperature for which the two maxima of $p_{N, \beta}(e)$ have the same height. Let us suppose that $\beta_{N, t}^{-1}$ converges to a fixed point $\bar{\beta}_{t}^{-1}$ as well as the two maximum energies $e_{N, \beta}^{(i)} \rightarrow \bar{e}_{\beta}^{(i)}$. For all temperature lower (higher) than $\bar{\beta}_{t}^{-1}$ only the low (high) energy peak will survive at the thermodynamic limit since the 
difference of the two maximum probabilities will be raised to the power $N$. This means that if $\bar{e}_{\beta}^{(1)} \neq \bar{e}_{\beta}^{(2)}$, the asymptotic canonical temperature $\bar{\beta}_{t}^{-1}$ corresponds to a discontinuity in the state energy, i.e. to a first order phase transition. This shows that the thermodynamic limit of a bimodal distribution is a first order phase transition, if the anomaly survives with increasing number of particles.

Let us now turn to the analysis of the microcanonical ensemble. For all canonical temperatures $\beta^{-1} \neq \bar{\beta}_{t}^{-1}$ the distribution $p_{N, \beta}(e)$ is asymptotically normal, meaning that the canonical and microcanonical caloric curves coincide in the thermodynamic limit for all energies below $\bar{e}_{\beta}^{(1)}$ and above $\bar{e}_{\beta}^{(2)}$ (see Sect. 6.1). However the canonic jump at $\bar{\beta}_{t}^{-1}$ is independent of the behaviour of the entropy between $\bar{e}_{\beta}^{(1)}$ and $\bar{e}_{\beta}^{(2)}$. This means that the backbending microcanonical caloric curve can a priori present any asymptotic behaviour in the canonical coexistence zone and does not necessarily converge to a plateau. In the particular case of short range interactions however, the Van Hove theorem discussed in Section 4.1 (Chap. 1) ensures that the backbending does indeed converge to a plateau; the case of long range interactions is postponed to Section 6.9.

\subsection{Equivalence between bimodalities and the Yang Lee theorem}

A more rigorous demonstration of the relation between bimodalities and first order phase transitions in the thermodynamic limit can be done with the help of the Yang Lee unit circle theorem [33]. The Yang Lee theorem [25] considers the distribution of the zeroes of the partition sum $Z_{\beta}$ in the complex $\beta$ plane. Under very general conditions it is possible to demonstrate $[25,34]$ that a first order phase transition corresponds to a distribution of zeroes for the partition sum of the finite system of size $N$, that asymptotically forms a line parallel to the imaginary axis and cuts across the real axis with a density increasing with the number of particles of the system, leading to a vanishing imaginary part for $N \rightarrow \infty$ (and to a non analyticity at a definite real value of $\beta$ ).

Let us first show that a bimodal distribution corresponds to a partition sum fulfilling the Yang Lee theorem in the double saddle point approximation $[28,35]$. The partition sum for a complex parameter $\lambda=\beta+i \eta$ is nothing but the Laplace transform of the probability distribution $P_{\beta_{0}}(e)$ for any temperature parameter $\beta_{0}[35,36]$

$$
Z_{\lambda}=\int d e Z_{\beta_{0}} P_{\beta_{0}}(e) e^{-\left(\lambda-\beta_{0}\right) e} \equiv \int d e p_{\beta}(e) e^{-i \eta e},
$$

where we have defined a (non normalized) distribution $p_{\beta}(e)=Z_{\beta} P_{\beta}(e)$. In order to study the thermodynamic limit (when it exists), if $p_{\beta}(e)$ is monomodal we can use a saddle point approximation around the maximum $\bar{e}$ giving $Z_{\lambda}=e^{\phi_{\lambda}(\bar{e})}$, with

$$
\phi_{\lambda}(e)=\log p_{\beta}(e)-i \eta e+\frac{1}{2} \eta^{2} \sigma^{2}(e)+\frac{1}{2} \log \left(2 \pi \sigma^{2}(e)\right),
$$


where $\sigma^{-2}=-\partial_{e}^{2} \log p_{\beta}(e)$. However, if the density of states $\bar{W}(e)=Z_{0} P_{0}(e)$ has a curvature anomaly it exists a range of $\beta$ for which the equation $\partial_{e} \log P_{\beta_{t}}(e)-\left(\beta-\beta_{t}\right)=0$ has three solutions $e_{1}, e_{2}$ and $e_{3}$. Two of these extrema are maxima so that we can use a double saddle point approximation which will be valid close to thermodynamic limit [35]

$$
Z_{\lambda}=e^{\phi_{\lambda}\left(e_{1}\right)}+e^{\phi_{\lambda}\left(e_{3}\right)}=2 e^{\phi_{\lambda}^{+}} \cosh \left(\phi_{\lambda}^{-}\right)
$$

where $2 \phi_{\lambda}^{+}=\phi_{\lambda}\left(e_{1}\right)+\phi_{\lambda}\left(e_{3}\right)$ and $2 \phi_{\lambda}^{-}=\phi_{\lambda}\left(e_{1}\right)-\phi_{\lambda}\left(e_{3}\right)$. The zeros of $Z_{\lambda}$ then correspond to $2 \phi_{\lambda}^{-}=i(2 n+1) \pi$. The imaginary part is given by $\eta=(2 n+1) \pi /\left(e_{3}-e_{1}\right)$ while for the real part we should solve the equation $\mathfrak{R}\left(\phi_{\lambda}^{-}\right)=0$. In particular, close to the real axis this equation defines a $\beta$ which can be taken as $\beta_{t}$. If the bimodal structure persists when the number of particles goes to infinity, the loci of zeros corresponds to a line perpendicular to the real axis with a uniform distribution as expected for a first order phase transition.

We can also work out the necessary condition and show that a uniform distribution of zeroes perpendicular to the real axis with a density linearly increasing with the number of particles $N$ implies a bimodal probability distribution [33]. Let us denote zeroes as $\lambda_{n}=\beta_{0}+i(2 n+1)(\pi / N \delta)$ such that the interval $2 \pi / \delta$ contains $N$ uniformly distributed zeroes in agreement with the unit circle theorem [25]. Since all the zeroes of $Z_{\lambda}$ are periodically distributed one can define an analytic function $f(\lambda)$ such that

$$
Z_{\lambda}=2 \cosh \left(\left(\lambda-\beta_{0}\right) N \delta / 2\right) f(\lambda) .
$$

On the line of zeroes, relation (49) reduces to $Z_{\beta_{0}+i \eta}=2 \cos (\eta N \delta / 2) f\left(\beta_{0}+i \eta\right)$. Using the fact that the partition sum along the line of zeroes is the Fourier transform of the reduced probability distribution at the transition point $\beta_{0}$ (Eq. (48)), we can write for the distribution

$$
p_{\beta_{0}}(e)=g_{\beta_{0}}(e+N \delta / 2)+g_{\beta_{0}}(e-N \delta / 2),
$$

where

$$
g_{\beta}(e)=\frac{1}{2 \pi} \int d \eta f(\gamma) e^{i \eta e}
$$

is a distribution. Indeed if we compute $p_{\beta}(e)$ a little above $\beta_{0}, \beta=\beta_{0}+\varepsilon$ we get

$$
p_{\beta_{0}+\varepsilon}(e)=e^{\varepsilon N \delta / 2} g_{\beta_{0}+\varepsilon}\left(e+\frac{N \delta}{2}\right)+e^{-\varepsilon N \delta / 2} g_{\beta_{0}+\varepsilon}\left(e-\frac{N \delta}{2}\right) .
$$

For large $N$ only the first term survives

$$
p_{\beta_{0}+\varepsilon}(e) \simeq e^{\varepsilon N \delta / 2} g_{\beta_{0}+\varepsilon}\left(e+\frac{N \delta}{2}\right) \equiv p_{\beta_{0}+\varepsilon}^{(1)}(e),
$$

which is the first term in the distribution at the transition point (Eq. (50)). If we conversely compute $p_{\beta}(e)$ a little below the transition point we get

$$
p_{\beta_{0}-\varepsilon}(e) \simeq e^{\varepsilon N \delta / 2} g_{\beta_{0}-\varepsilon}\left(e-\frac{N \delta}{2}\right) \equiv p_{\beta_{0}-\varepsilon}^{(2)}(e),
$$


which is the second term in the transition point distribution (Eq. (50)). At the transition point $p_{\beta_{0}}(e)$ is the sum of two shifted (identical) distributions. If $g_{\beta}$ is a normal (i.e. monomodal) distribution, the central limit theorem guarantees that for a large number of particles its width will scale as $\sqrt{N}$, i.e. will grow slower than the distance between the two peaks that scales as $N$. This implies that (for not too small $N) p_{\beta_{0}}(e)$ is bimodal, the distance $\delta$ between the two peaks representing asymptotically the latent heat per particle.

This demonstration can be extended to account for more important finite size effects [33], when zeroes $\lambda_{n}$ are already periodic but not yet uniformly distributed on a straight line $[35,37]$.

In this section we have demonstrated that the asymptotic bimodality of the order parameter distribution function is a necessary and sufficient condition for a first order phase transition in the thermodynamic limit. There is however a very important difference between finite systems and the thermodynamic limit: in the latter case the probability distribution is bimodal only at the transition point $\beta_{0}$ while in finite systems, an interval $\Delta \beta$ of non-zero measure exists where the two phases coexist, i.e. the distribution is bimodal, each peak being associated with a phase having a finite probability of occurrence. As we will see in Section 4.1 (Chap. 2), the extension of the transition point to a finite transition region makes a direct measurement of phase coexistence possible [38], contrary to the common belief that phase transitions would only be loosely defined out of the thermodynamic limit. Moreover, the fact that the distribution is non zero between the two maxima in the case of small systems implies that microstates can be accessed that do not exist at the thermodynamic limit in the intensive ensemble. These states are specific of the coexistence region and can lead to spectacular phenomena as negative heat capacity [12] and negative compressibility [11] in the extensive ensemble.

\subsection{Ensemble inequivalence up to the thermodynamic limit}

Let us now investigate the more general situation when the system size goes to infinity and the Van Hove theorem (Sect. 4.1, Chap. 1) does not necessarily apply. We have already observed in the last section that the asymptotic behaviour of an ensemble that explores the coexistence region of a first order phase transition cannot be predicted from the thermodynamic limit of the corresponding intensive ensemble, for the simple reason that this latter is not defined inside coexistence. In particular the microcanonical caloric curve in the phase transition region may either converge towards the Maxwell construction, or keep a backbending behaviour, since a negative heat capacity system can be thermodynamically stable even in the thermodynamic limit if it is isolated. Indeed we have shown in Section 5 (Chap. 1) that if the interaction is long ranged, the topological anomaly leading to the convex intruder in the entropy is not cured by increasing the number of particles $[21,39,40]$.

Within our approach based on the topology of the probability distribution of observables [28], we have demonstrated that ensemble inequivalence arises from 
fluctuations of the order parameter. Ensembles putting different constraints on the fluctuations of the order parameter lead to different thermodynamics. In the case of phase transitions with non-zero latent heat, the total energy usually plays the role of an order parameter except if energy fluctuations are forbidden; therefore, the microcanonical ensemble is expected to present a different thermodynamics than the other (canonical) ensembles. This different behaviour may remain at the thermodynamic limit, and this frequently occurs for Hamiltonians containing a kinetic energy contribution: in this case we will show that the microcanonical caloric curve can present at the thermodynamic limit a temperature jump in complete disagreement with the canonical ensemble [41].

Let us consider a finite system for which the Hamiltonian can be separated into two components $E=E_{1}+E_{2}$, that are statistically independent $\left(W\left(E_{1}, E_{2}\right)=\right.$ $\left.W_{1}\left(E_{1}\right) W_{2}\left(E_{2}\right)\right)$ and such that the associated degrees of freedom scale in the same way with the number of particles; we will also consider the case where $S_{1}=\log W_{1}$ has no anomaly while $S_{2}=\log W_{2}$ presents a convex intruder which is preserved at the thermodynamic limit. A typical examples of $E_{1}$ is given by the kinetic energy for a classical system with velocity independent interactions. For other similar one-body operators see reference [39].

The probability to get a partial energy $E_{1}$ when the total energy is $E$ is given by

$$
P_{E}\left(E_{1}\right)=\exp \left(S_{1}\left(E_{1}\right)+S_{2}\left(E-E_{1}\right)-S(E)\right) .
$$

The extremum of $P_{E}\left(E_{1}\right)$ is obtained for the partitioning of the total energy $E$ between the kinetic and potential components that equalizes the two partial temperatures $\bar{T}_{1}^{-1}=\partial_{E_{1}} S_{1}\left(\bar{E}_{1}\right)=\partial_{E_{2}} S_{2}\left(E-\bar{E}_{1}\right)=\bar{T}_{2}^{-1}$. If $\bar{E}_{1}$ is unique, $P_{E}\left(E_{1}\right)$ is mono-modal and we can use a saddle point approximation around this solution to compute the entropy $S(E)=\log \int_{-\infty}^{E} d E_{1} \exp \left(S_{1}\left(E_{1}\right)+S_{2}\left(E-E_{1}\right)\right)$. At the lowest order, the entropy is simply additive so that the microcanonical temperature of the global system $\partial_{E} S(E)=\bar{T}^{-1}$ is the one of the most probable energy partition. Therefore, the most probable partial energy $\bar{E}_{1}$ acts as a microcanonical thermometer. If $\bar{E}_{1}$ is always unique, the kinetic thermometer in the backbending region will follow the whole decrease of temperature as the total energy increases. Therefore, the total caloric curve will present the same anomaly as the potential one.

If conversely the partial energy distribution is double humped, then the equality of the partial temperatures admits three solutions, one of them $\bar{E}_{1}^{(0)}$ being a minimum. At this point the partial heat capacities $C_{1}^{-1}=-\bar{T}^{2} \partial_{E_{1}}^{2} S_{1}\left(\bar{E}_{1}^{(0)}\right)$ and $C_{2}^{-1}=-\bar{T}^{2} \partial_{E_{2}}^{2} S_{2}\left(E-\bar{E}_{1}^{(0)}\right)$ fulfill the relation

$$
C_{1}^{-1}+C_{2}^{-1}<0
$$

This happens when the potential heat capacity is negative and the kinetic energy is large enough $\left(C_{1}>-C_{2}\right)$ to act as an approximate heat bath: the partial energy distribution $P_{E}\left(E_{1}\right)$ in the microcanonical ensemble is then bimodal as the total 
energy distribution $P_{\beta}(E)$ in the canonical ensemble. The bimodality of $P_{E}\left(E_{1}\right)$ implies that the kinetic energy is an order parameter of the transition in the microcanonical ensemble. This is almost a paradox since in any other ensembles in which no total energy conservation is imposed the kinetic energy has a trivial perfect gas behaviour, while in the microcanonical ensemble it becomes an order parameter with the specific bimodal structure at the phase transition.

In this case, performing a double saddle point approximation around the two maxima leads to a microcanonical temperature given by a weighted average of the two estimations from the two maxima of the kinetic energy distribution

$$
T=\partial_{E} S(E)=\frac{\bar{P}^{(1)} \sigma^{(1)} / \bar{T}^{(1)}+\bar{P}^{(2)} \sigma^{(2)} / \bar{T}^{(2)}}{\bar{P}^{(1)} \sigma^{(1)}+\bar{P}^{(2)} \sigma^{(2)}}
$$

where $\bar{T}^{(i)}=T_{1}\left(\bar{E}_{1}^{(i)}\right)$ are the kinetic temperatures calculated at the two maxima, $\bar{P}^{(i)}=P_{E}\left(\bar{E}_{1}^{(i)}\right)$ are the probabilities of the two peaks, and $\sigma^{(i)}$ their widths. At the thermodynamic limit (Eq. (52)) reads $c_{1}^{-1}+c_{2}^{-1}<0$, with $c=\lim _{N \rightarrow \infty} C / N$. If this condition is fulfilled the probability distribution $P_{E}\left(E_{1}\right)$ presents two maxima for all finite sizes and only the highest peak survives at $N=\infty$. Let $E_{t}$ be the energy at which $P_{E_{t}}\left(\bar{E}_{1}^{(1)}\right)=P_{E_{t}}\left(\bar{E}_{1}^{(2)}\right)$. Because of equation (53) at the thermodynamic limit the caloric curve will follow the high (low) energy maximum of $P_{E}\left(E_{1}\right)$ for all energies below (above) $E_{t}$; there will be a temperature jump at the transition energy $E_{t}$.

Let us illustrate the above results with two examples for a classical gas of interacting particles. For the kinetic energy contribution we have $S_{1}(E)=c_{1} \ln (E / N)^{N}$ with a constant kinetic heat capacity per particle $c_{1}=3 / 2$. For the potential part we will take two polynomial parametrizations of the interaction caloric curve presenting a back bending which are displayed in the left part of Figure 27. If the decrease of the partial temperature $T_{2}\left(E_{2}\right)$ is steeper than $-2 / 3$ (Fig. 27a) [21], equation (52) is verified and the kinetic caloric curve $T_{1}\left(E-E_{1}\right)$ (dot-dashed line) crosses the potential one $T_{2}\left(E_{2}\right)$ (full line) in three different points for all values of the total energy lying inside the coexistence region. The resulting caloric curve for the whole system is shown in Figure 27b (symbols) together with the thermodynamic limit (lines) evaluated from the double saddle point approximation (53). In this case one observes a temperature jump at the transition energy. If the temperature decrease is smoother (Fig. 27c) the shape of the interaction caloric curve is preserved at the thermodynamic limit (Fig. 27d).

This patent violation of ensemble equivalence means that, contrary to the physical intuition based on macro-systems, the equations of state explicitly depend on the characteristics of the considered ensemble of events i.e. on the state variables, namely the fluctuating observables and the conserved quantities imposed by the dynamics or by the sorting technique. This implies the impossibility to define a unique thermodynamics, i.e. a unique equation of state, for systems undergoing a first order phase transition. 


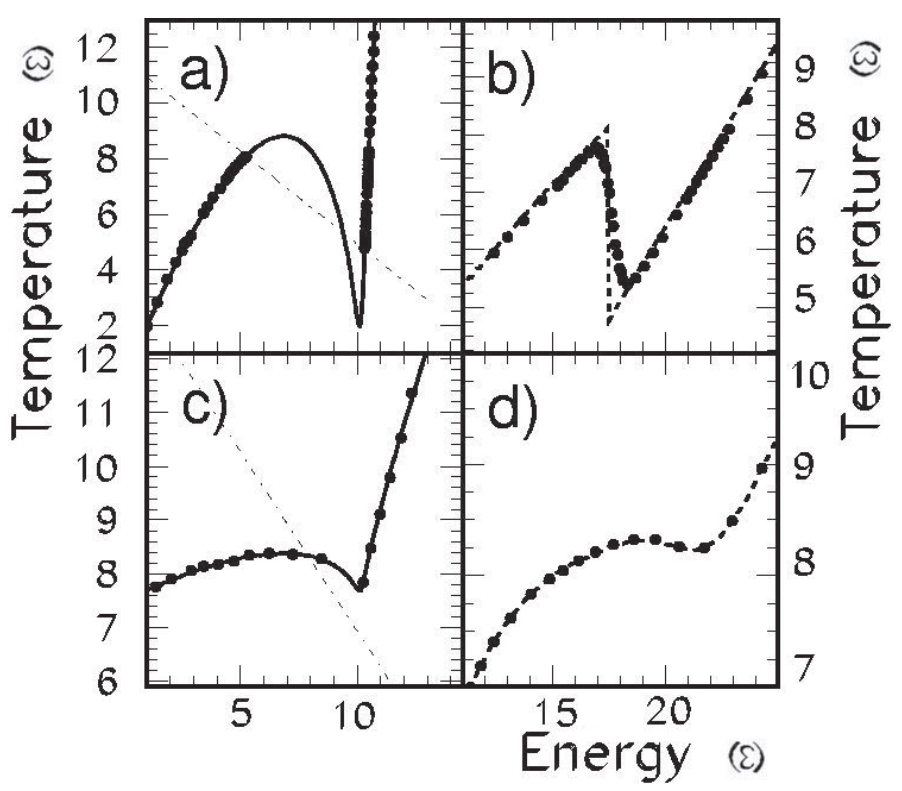

Figure 27. Left panels: temperature as a function of the potential energy $E_{2}$ (full lines) and of the kinetic energy $E-E_{2}$ (dot-dashed lines) for two model equation of states of classical systems showing a first order phase transition. Symbols: temperatures extracted from the most probable kinetic energy thermometer from equation (51). Right panels: total caloric curves (symbols) corresponding to the left panels and thermodynamic limit of equation (53) (dashed lines).

\section{First order phase transitions and abnormal fluctuations}

In the previous section we have seen some model cases in which the kinetic energy is bimodal in the coexistence region of the microcanonical ensemble. Indeed the potential energy is an order parameter for phase transitions with non zero latent heat; the microcanonical constraint creates a one to one correlation between the kinetic and potential components, and this leads to a microcanonical bimodal kinetic distribution. For most model Hamiltonians however, the potential energy distribution is strongly constrained by the requirement of total energy conservation and the bimodality in $E_{\text {pot }}$ is suppressed under the microcanonical constraint.

A similar example has been already presented in Section 6.6 (Chap. 1), where the bimodality of the $A_{\max }$ distribution in the coexistence zone of the liquid gas phase transition was suppressed by the canonical constraint of total mass conservation because of the correlation between $A_{\max }$ and the total mass. However, even if the distribution is not bimodal in the extensive ensemble $A_{\text {tot }}=c s t$., the domain of variation of $A_{\max }$ is still extremely large: we can then expect that the distributions width may bear some information about the possible occurrence of 
a phase coexistence even in an ensemble where a conservation law applies to a variable directly correlated to the order parameter.

Let us come back to the microcanonical ensemble. If the Hamiltonian of the system is known, the presence (or absence) of a double hump in the partial energy distribution can be predicted looking at the difference between the kinetic and potential heat capacities (Sect. 6.9, Chap. 1), but in the case of short range forces the Van Hove theorem guarantees that the kinetic distribution has to be (at least asymptotically) normal. In this case we can perform a simple saddle point approximation and we will show in Section 7.1 that the width of the partial energy distribution is a direct measure of the microcanonical heat capacity, and that an isolated system undergoing a first order phase transition can be represented as composed of subsystems with abnormally large fluctuations $[42,45]$.

The presence of abnormal fluctuations can be thus taken as a definition of phase transition equivalent (within an approximation discussed in Sect. 7.2) to the definitions that we have introduced earlier in this paper. In the specific case of a multidimensional observables space (as for example in the liquid gas phase transition) the fluctuation observable has the extra advantage that it is independent of the different possible transformations and it is directly connected to the underlying density of states. This point will be discussed in Section 7.3.

\subsection{Partial energy fluctuations and heat capacity}

Let us consider, as in Section 6.9 above, a system which can be decomposed into two independent components so that the density of states is factorized $W\left(E_{1}, E_{2}\right)=$ $W_{1}\left(E_{1}\right) W_{2}\left(E_{2}\right)$ and the total energy is the sum of the two partial energies $E_{t}=$ $E_{1}+E_{2}$. In a microcanonical ensemble with a total energy $E_{t}$, the total state density $W\left(E_{t}\right)=\exp \left(S\left(E_{t}\right)\right)$ is given by the folding of the partial state densities $W_{i}\left(E_{i}\right)$, $S\left(E_{t}\right)=\log \int_{0}^{E} d E_{1} \exp \left(S_{1}\left(E_{1}\right)+S_{2}\left(E-E_{1}\right)\right)$. The energy distribution in system (1) when the total energy is $E_{t}$ is (see Eq. (51))

$$
P_{1}\left(E_{1}\right)=\frac{W_{1}\left(E_{1}\right) W_{2}\left(E_{t}-E_{1}\right)}{W\left(E_{t}\right)}=\exp \left(S_{1}\left(E_{1}\right)+S_{2}\left(E_{t}-E_{1}\right)-S\left(E_{t}\right)\right) .
$$

If this distribution is double humped, the system is undergoing a first order phase transition with negative heat capacity that may converge to a temperature jump in the thermodynamic limit (see Sect. 6.9). If on the other hand this distribution is normal, we can make a single saddle point approximation for the partition sum of system (1) (say, the kinetic energy) which corresponds to a Gaussian approximation for the distribution [45], $P_{1}\left(E_{1}\right)=\left(2 \pi \sigma^{2}\right)^{-1 / 2} \exp \left(-\left(E_{1}-\bar{E}_{1}\right)^{2} / 2 \sigma^{2}\right)$. Substituting into equation (54) gives

$$
\frac{1}{\sigma^{2}}=-\left.\frac{\partial^{2} S_{1}}{\partial E_{1}^{2}}\right|_{\bar{E}_{1}}-\left.\frac{\partial^{2} S_{2}}{\partial E_{2}^{2}}\right|_{E_{t}-\bar{E}_{1}}=\left(\frac{\partial^{2} \log Z_{1}}{\partial \beta^{2}}\right)^{-1}-\frac{1}{T^{2} C_{2}},
$$

where $T$ is the microcanonical temperature

$$
T^{-1}=\partial S_{1} / \partial E_{1}\left(\bar{E}_{1}\right)=\partial S_{2} / \partial E_{2}\left(E_{t}-\bar{E}_{1}\right)
$$


associated with the most probable partitioning of the total energy $E_{t}$ between the two subsystems, $C_{2}$ is the heat capacity of the interacting part, $C_{2}^{-1}=$ $-T^{2} \partial^{2} S_{2} / \partial E_{2}^{2}\left(\bar{E}_{2}\right), Z_{1}$ is the canonical partition sum for system (1), and the second equality stems from the fact that in the saddle point approximation the Legendre and Laplace transforms coincide (see Sect. 2.1, Chap. 1). In the same saddle point approximation the heat capacity are simply additive $C=C_{1}+C_{2}$, which gives for the total microcanonical heat capacity

$$
C=\frac{C_{1}^{2}}{C_{1}-\frac{\sigma^{2}}{T^{2}}} .
$$

Equation (56) has been first derived by Lebowitz in reference [42] (see also Ref. [43]) and has not to be confused with the (similar) exact expressions of references [44]. In the next section we will show that the finite size corrections to equation (56) are generally negligible already for systems composed of a few tens of particles. The advantage of equation (56) respect to the expressions of reference [44] is that equation (56) can be applied to different sharings of the conserved extensive variable and does not require that $S_{1}\left(E_{1}\right)$ represents the entropy of a free classical gas. This will be of a crucial importance in the application to experimental data, as we will see in Section 2 (Chap. 2).

Since in the canonical ensemble the energy fluctuation is a direct measure of the heat capacity, $\partial^{2} \log Z_{1} / \partial \beta^{2}=\sigma_{c a n}^{2}$, we can also write for the interaction part

$$
C_{2}^{-1}=T^{2}\left(\frac{1}{\sigma^{2}}-\frac{1}{\sigma_{c a n}^{2}}\right),
$$

and for the heat capacity of the total system

$$
\left(\frac{C}{C_{1}}\right)^{-1}=1-\frac{\sigma^{2}}{\sigma_{c a n}^{2}}
$$

Equation (57) shows that a negative heat capacity corresponds to partial energy fluctuations in the microcanonical ensemble that exceed the corresponding fluctuations in the canonical ensemble.

We are here confronted with another paradox connected to negative heat capacity: the implementation of a constraint $E_{\text {tot }}=c s t$. produces an increase of fluctuations on a variable $E_{1}$ correlated to the constraint, respect to the canonical case where this same variable was free to fluctuate. The physical origin of this strange behaviour is easily understood if we recall that in the microcanonical ensemble the fluctuations of the two subsystems are identical $\sigma_{1}^{2}=\sigma_{2}^{2}$. The potential energy presents huge fluctuations because the system is jumping between the two phases and the microcanonical constraint forces the kinetic energy to compensate this fluctuation. 


\subsection{Finite size corrections}

Even if the interactions are short ranged and therefore the kinetic energy distribution is normal, one may doubt about the validity of the Gaussian approximation employed in the last section, especially if we deal with very small systems. The first order correction to the saddle point approximation for the entropy is easily computed [45]

$$
S\left(E_{t}\right)=S_{1}\left(\bar{E}_{1}\right)+S_{2}\left(E_{t}-\bar{E}_{1}\right)+\frac{1}{2} \ln \left(2 \pi \sigma^{2}\right) .
$$

Looking now at the first derivative of $S$ we get

$$
\frac{1}{T}=\frac{1}{\bar{T}}+\frac{\partial \ln \sigma}{\partial E_{t}}
$$

where $\bar{T}$ is the most probable temperature of the partial systems given by equation (55). The second derivative of the entropy allows to compute the heat capacity

$$
C^{-1}=\left(1-T \frac{\partial \ln \sigma}{\partial E_{t}}\right)^{2}\left(\bar{C}^{-1}-\frac{1}{\bar{T}^{2}} \frac{\partial^{2} \ln (\sigma)}{\partial E_{t}^{2}}\right),
$$

where $\bar{C}$ represents the saddle point approximation (Eq. (56)). At zero order in the kinetic energy variance $\sigma$ we recover the simple result $C=\bar{C}$, however in general we will have to consider corrections to this relation due to the microcanonical fluctuations.

In practical applications of equation (56), the validity of this approximation has to be verified by explicitly testing the quality of a Gaussian fit on the partial energy distribution, and by computing the correcting terms equation (58). An example is given in Figure 28 for a system of 216 particles in the isobar lattice gas model at a subcritical pressure. The heat capacity extracted from the kinetic energy fluctuations with the expression at zero order, equation (56), is already in very good agreement with the exact one. This means that kinetic energy fluctuations are an experimentally accessible measure of the heat capacity, which allows to sign divergences and negative branches characteristic of the phase transition.

It is also interesting to note that equation (57) can be extended to a more general equilibrium situation. Consider for a first order phase transition a system characterized by an observable (order parameter) $A_{\text {tot }}$ that can be divided into two subsystems $A_{\text {tot }}=A+A_{0}$ (say: fragments and monomers in the liquid gas phase transition) such that no anomaly is present in the density of states of $A_{0}$ and the two systems are statistically independent. Define a generalized susceptibility $\chi^{-1}=-\partial^{2} S / \partial A^{2}$. The transition is then characterized by a negative branch of $\chi$ according to the equation

$$
\chi^{-1}=\frac{1}{\sigma^{2}}-\frac{1}{\sigma_{c a n}^{2}},
$$

where $\sigma^{2}$ is the fluctuation of $A_{0}$ under the constraint $A_{\text {tot }}=$ cst. and $\sigma_{\text {can }}^{2}$ is the same fluctuation when the constraint is relaxed. 


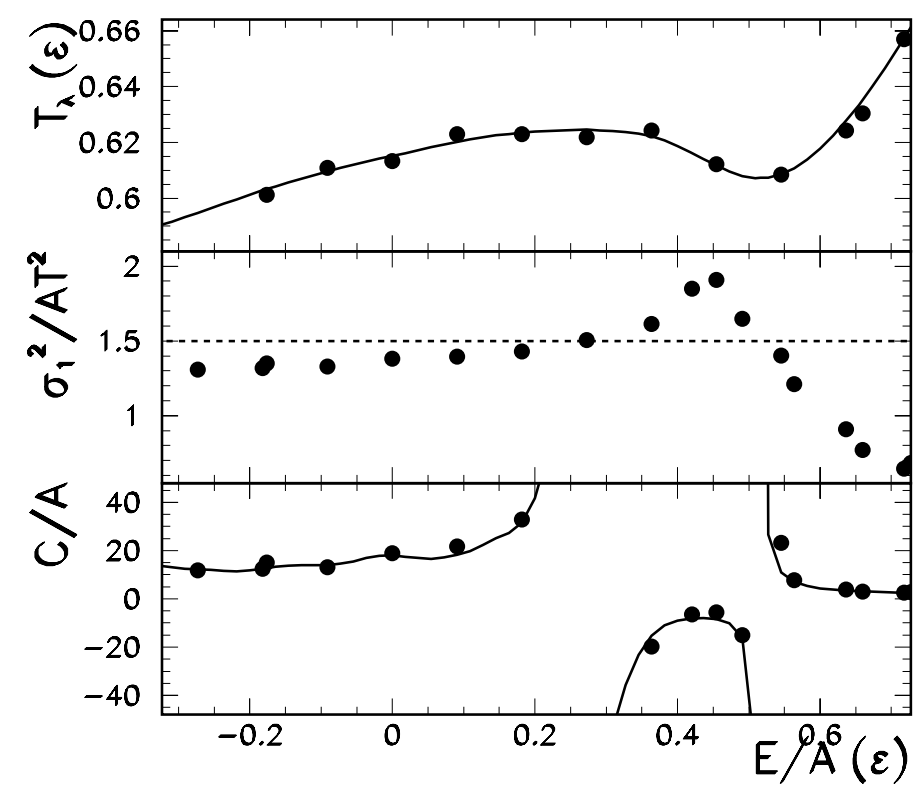

Figure 28. As a function of the total energy per particle are shown: (a) the average kinetic energy (dots) multiplied by $2 / 3$ compared with the microcanonical temperature (line); (b) the kinetic energy fluctuations (dots) compared with the canonical expectation (line); (c) the heat capacity extracted from the fluctuations (dots) compared with the exact one (line).

\subsection{State variables versus transformations}

When dealing with finite systems for which the different statistical ensembles are not equivalent, it is essential to distinguish between state variables, which only depend on the underlying equations of state, and transformations, which also depend on the thermodynamic path followed by the system.

Let us take the example of the liquid gas phase transition, for which the energy $E$, the volume $V$ and the number of particles $A$ are all relevant observables (indeed they are all order parameters in the corresponding intensive ensembles). The system is then characterized by (at least) three state variables, that identify the statistical ensemble. For instance $\left(\beta, \lambda_{V}, A\right)$ defines the canonical isobar ensemble with partition sum

$$
Z_{\beta p}(A)=\sum_{V} \sum_{E} W(E, A, V) \exp \left(-\beta E-\lambda_{V} V\right)
$$

with $p=\lambda_{V} / \beta ;\left(E, \lambda_{V}, A\right)$ corresponds to the microcanonical iso- $\lambda_{V}$ ensemble (see Sect. 6.4, Chap. 1)

$$
Z_{\lambda_{V}}(E, A)=\sum_{V} W(E, A, V) \exp \left(-\lambda_{V} V\right)
$$


$(E, V, A)$ defines the microcanonical isochore ensemble where the partition sum is given by the density of states $Z(E, A, V) \equiv W(E, A, V)$, etc.

Any other state variable that can be computed to characterize the system (for instance the temperature $T$ in a microcanonical ensemble) will then be described by a function in a three dimensional space. The temperature $T_{\lambda_{V}}^{-1}=\partial Z_{\lambda_{V}} / \partial E$ in the microcanonical iso- $\lambda_{V}$ ensemble is shown in Figure 29 for the lattice gas model. The anomalous curvature related to the first order phase transition (see Sect. 4, Chap. 1) is apparent. In Section 4 we have analyzed the transition looking at the backbending of the caloric curve; however any curve defined on the two dimensional temperature surface of Figure 29 can be a caloric curve. This means that if the state variables are more than one, then the behaviour of the caloric curve depends on the transformation and does not bear information on the underlying partition sum. As an example the behaviour of the temperature as a function of energy at a constant pressure $\left(p=\lambda_{V} T\right)$ or a constant average volume in the subcritical region are displayed in the upper part of Figure 30. At constant pressure the caloric curves are similar the ones at constant $\lambda_{V}$ presented in Figure 29, and a backbending is clearly seen. On the other hand at constant average volume a smooth behaviour is observed, as expected from general thermodynamics. From these examples it is clear that the backbending of the temperature surface can be

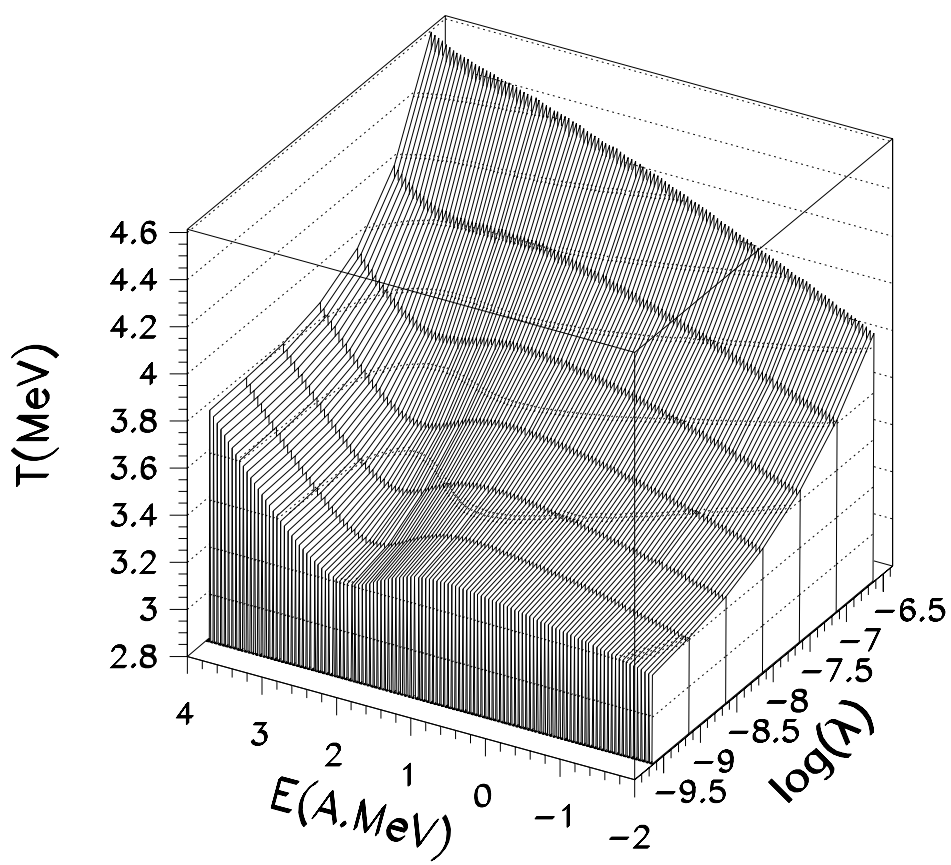

Figure 29. Temperature as a function of the energy per particle and the Lagrange parameter associated to volume for a lattice gas system of 216 particles in the microcanonical ensemble with fluctuating volume. The coupling $\epsilon$ is fixed to $5.5 \mathrm{MeV}$. 


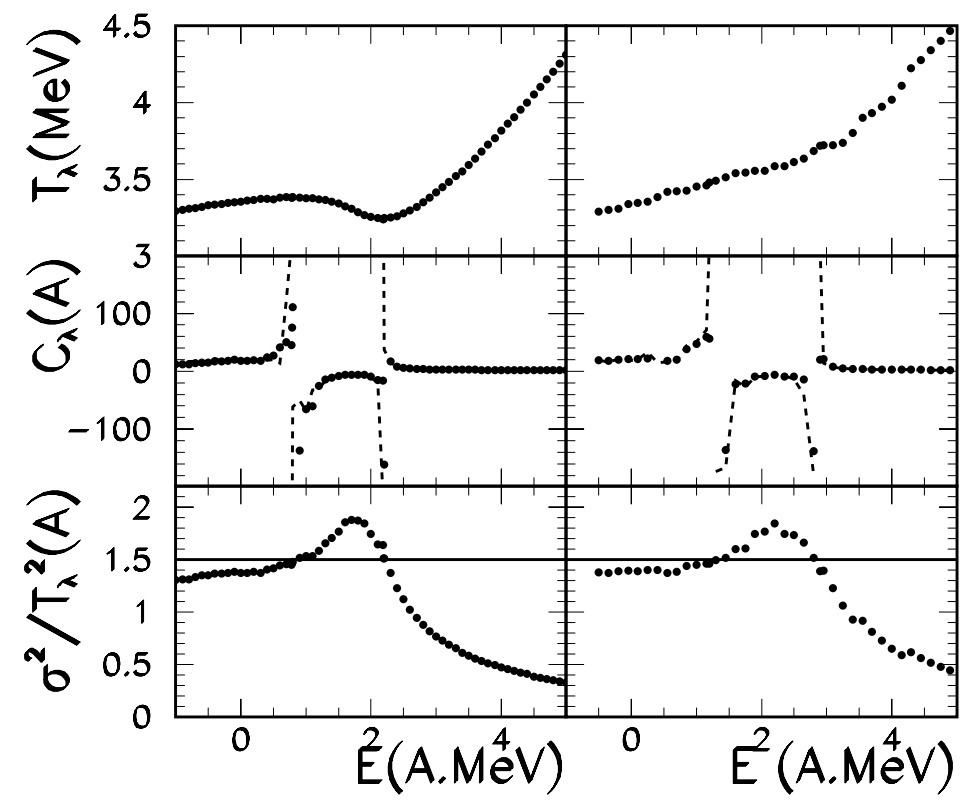

Figure 30. Thermodynamic quantities in the microcanonical ensemble for a transformation at constant pressure (left part) and at constant volume (right part). Upper panels: caloric curve. Lower panels: normalized kinetic energy fluctuations compared to the canonical expectation (lines). Medium panels: heat capacity $C_{\lambda}$ (symbols) compared to the estimation through equation (56) (lines). The coupling $\epsilon$ is fixed to $5.5 \mathrm{MeV}$.

avoided depending on the path of the considered transformation, and the phase transition signal can be hidden in the observation of the caloric curve.

On the other side partial energy fluctuations are a state variable which does not depend on the transformation from one state to another. Figure 31 shows the fluctuation contour plot in the $(\lambda, E)$ plane. The level corresponding to the reference value $\sigma^{2} / T^{2}=1.5$ is shown. The heat capacity diverges along the isocontour labelled by 1.5, while inside the region delimited by this curve the normalized fluctuation exceeds 1.5, which corresponds to a negative heat capacity. The 1.5 isocontour is equivalent to the spinodal curve as it can be seen from the $\lambda(E)$ isotherms shown as dashed lines. Indeed the region delimited by the isocontour $\sigma^{2} / T^{2}=1.5$ coincides with the locus of the points of inverted slope for the isotherms. The critical temperature is indicated by the thickest dashed line: it is clear that the endpoint of the 1.5 isocontour coincides with the thermodynamic critical point.

From Figure 31 we can see that, in agreement with the results of Section 7.1 (Chap. 1), in the whole phase transition region the microcanonical fluctuations present a strong maximum which exceeds the canonical value in the spinodal region: an anomalously large fluctuation signal will be always seen if the system undergoes a first order phase transition, independent of the path. As an example 


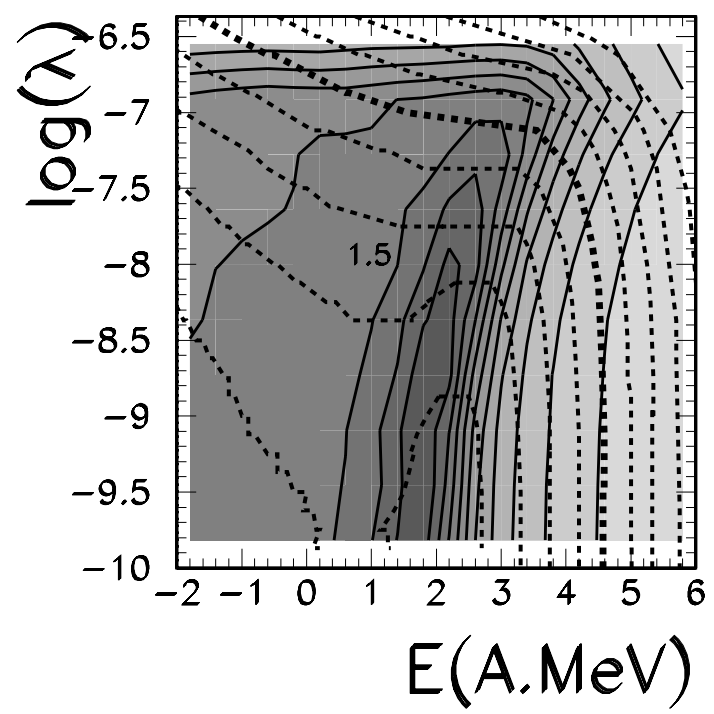

Figure 31. Isotherms (dashed lines) and contour plot of the normalized kinetic energy fluctuations in the Lagrange parameter versus energy plane. The level corresponding to the canonical expectation $\sigma^{2} / T^{2}=1.5$ is shown. Thick dashed line: critical isotherm. The coupling $\epsilon$ is fixed to $5.5 \mathrm{MeV}$.

the lower part of Figure 30 shows a constant $p$ or $\langle V\rangle$ cut of the bidimensional fluctuation surface shown in Figure 31. The quantitative behaviour of the heat capacity as a function of energy depends on the specific transformation, but at each point the heat capacity extracted from fluctuations is a direct measure of the underlying equation of state. This is quantitatively demonstrated in the medium part of Figure 30. In this figure the heat capacity extracted from the equation of state, equation (60) (symbols)

$$
C_{\lambda_{V}}^{-1}=\partial T_{\lambda_{V}} / \partial E=-T_{\lambda_{V}}^{2} \partial^{2} Z_{\lambda_{V}} / \partial E^{2}
$$

are in very good agreement with the fluctuation estimation, equation (56) (lines).

This result is another consequence of the inequivalence between the different statistical ensembles. Since ensembles are not equivalent, there is no guarantee that a caloric curve at constant average volume in the iso- $\lambda_{V}$ ensemble $\left.T_{\lambda_{V}}(E)\right|_{\langle V\rangle}$ coincides with the isochore caloric curve $T_{V}(E)$ (i.e. the caloric curve in an ensemble where all microstates correspond to the same volume). The same reasoning applies to all state variables: for instance the response function $\left.C_{\lambda_{V}}(E)\right|_{\langle V\rangle}$ at constant average volume in the iso- $\lambda_{V}$ ensemble does not need to be equal to the isochore response function $C_{V}(E)$. Indeed Figure 30 shows that the heat capacity diverges in the iso- $\lambda_{V}$ ensemble even within a constant average volume transformation, while the isochore heat capacity $C_{V}$ is a smooth curve for any number of particles [46]. 
If the system crosses the coexistence region following a constant average volume transformation (right part of Fig. 31), the convexity anomaly of the entropy (Fig. 29) is not apparent in the caloric curve, yet the heat capacity shows a negative region between two divergencies. Indeed as soon as the system has more than one state variable, the heat capacity does not coincide with the derivative of the caloric curve. This latter is given by

$$
\frac{d T}{d E}=\left.\frac{\partial T}{\partial E}\right|_{\lambda_{V}}+\left.\frac{\partial T}{\partial \lambda_{V}}\right|_{E} \frac{\partial \lambda_{V}}{\partial E}=C_{\lambda_{V}}^{-1}+\left.\frac{\partial T}{\partial \lambda_{V}}\right|_{E} \frac{\partial \lambda_{V}}{\partial E}
$$

The heat capacity, exactly as the temperature, is a state variable, while the derivative of the caloric curve explicitly depends on the specific transformation $\lambda_{V}(E)$ employed. In a constant pressure transformation $\partial_{E} \lambda_{V}=p \partial_{E} \beta$ is very small since the temperature is almost constant in the isobar canonical ensemble (recall $\partial_{E} \lambda_{V}=0$ in the thermodynamic limit). In this case $d T / d E$ is a good estimation of the heat capacity $\partial T / \partial E$. On the other hand in a constant average volume transformation the constraining $\lambda_{V}$ has to variate rapidly with energy to compensate the sudden volume increase due to the appearance of the gas phase, and the derivative of the caloric curve will differ considerably from the heat capacity.

This means that the information on the convexity properties of the entropy can only be traced back through the measurement of a state variable, i.e. the fluctuation observable discussed in Section 7 (Chap. 1).

\subsection{What is temperature? (II)}

We have seen in Section 4.7 (Chap. 1) that the microcanonical relation

$$
T^{-1}=\frac{d \ln W(E)}{d E}
$$

is a measure of the physical temperature. After the discussion of the previous section, one may wonder if the generalization of this relation to the multidimensional state variables case is unique. No ambiguity exists from the statistical mechanics point of view: temperature being the Lagrange conjugate of the system energy for any generic Gibbs equilibrium, the expressions used in the previous section for the temperature and the heat capacity immediately follow

$$
\begin{aligned}
T^{-1} & =\frac{\partial \ln Z_{\lambda_{1}, \ldots, \lambda_{L}}\left(E, B_{1}, \ldots, B_{K}\right)}{\partial E} \\
C^{-1} & =-T^{2} \frac{\partial^{2} \ln Z_{\lambda_{1}, \ldots, \lambda_{L}}\left(E, B_{1}, \ldots, B_{K}\right)}{\partial E^{2}}=\frac{\partial T}{\partial E},
\end{aligned}
$$

where $\lambda_{i}, B_{i}$ represent the intensive and extensive variables controlled in the considered statistical ensemble. However the second law of thermodynamics $d S=\delta Q / T$ associates temperature to a variation of heat, and the relation $\delta Q=d E$ that we have used in Section 4.7 (Chap. 1) is correct only if no work is done on 
the system, i.e. in the isochore ensemble, while in the isobar ensemble one should rather use $\delta Q=d H$ where $H=E+p V$ is the free enthalpy.

It is easy to show that there is no contradiction between the statistical mechanics and the macroscopic thermodynamics points of view. Indeed if we consider a thermometer inside a system at constant pressure (see Sect. 4.7, Chap. 1), the energy and enthalpy distribution of the thermometer can be written as

$$
\begin{aligned}
& p_{\text {th }}(E)=\int \frac{d V}{Z} W_{t h}(E, V) e^{-\beta E-\lambda_{V} V} \equiv \frac{Z_{\lambda_{V}}^{\text {th }}(E)}{Z} e^{-\beta E} \\
& p_{\text {th }}(H)=\int \frac{d V}{Z} W_{t h}\left(H-\frac{\lambda_{V} V}{\beta}, V\right) e^{-\beta H} \equiv \frac{Z_{p}^{\text {th }}(H)}{Z} e^{-\beta H}
\end{aligned}
$$

where the relationship between the pressure and the Lagrange conjugate to the volume is given as usual by $\lambda_{V}=\beta p$. The response of the thermometer is given by its most probable state $\bar{E}, \bar{H}$

$$
\beta=\left.\frac{d}{d E} \log Z_{\lambda_{V}}^{\text {th }}(E)\right|_{\bar{E}}=\left.\frac{d}{d H} \log Z_{p}^{t h}(H)\right|_{\bar{H}^{\prime}}
$$

where thermal equilibrium between the thermometer and the system implies (see Eq. (39))

$$
\begin{aligned}
& \left.\frac{d \ln Z_{\lambda_{V}}^{\text {th }}}{d E}\right|_{\bar{E}}=\frac{d \ln Z_{\lambda_{V}}^{s y s}}{d E} \\
& \left.\frac{d \ln Z_{p}^{\text {th }}}{d H}\right|_{\bar{H}}=\frac{d \ln Z_{p}^{s y s}}{d H},
\end{aligned}
$$

in agreement with equation $(61)$. Equations $(63,64)$ show that the definitions $(61$, 62) are the correct definitions of the physical temperature and heat capacity for any statistical ensemble.

In the particular case of an ideal classical gas $p V=T$ the volume is exactly the inverse of the $\lambda_{V}$ Lagrange parameter, which leads to the result of Section 7.1 (Chap. 1) for the kinetic energy

$$
C_{\lambda_{V}}^{k i n}=C_{V}^{k i n}=3 / 2 .
$$




\section{Applications to nuclear multifragmentation}

Since nuclear forces resemble to Van der Waals interactions, the nuclear phase diagram is expected to present a liquid gas phase transition. Our present knowledge of the nuclear equation of state is limited. The main reason is the difficulty to treat the nuclear many-body problem and to define a reliable in medium interaction. The saturation energy and density, i.e. the ground state of nuclear matter, are well established but as far as the temperature dependence of nuclear properties is concerned, very little is known in an absolute way. Only the entropy variation, i.e. the level density parameter $a=S / T$, of a finite nucleus has been clearly established at low excitation energies through evaporation studies.

An important research activity is now devoted to the extraction of reliable information of the nuclear equation of states and the associated phase diagram. Heavy ion reactions are routinely used to test mechanical and thermodynamic properties of nuclei. In particular in the recent years the multifragmentation regime has been tentatively associated with the occurrence of a liquid-gas phase transition. In this context the fluctuation observable discussed in Section 7 (Chap. 1) represents a powerful tool to quantitatively assess the properties of the nuclear equation of state at finite temperature. Indeed $4 \pi$ detectors give an (almost) complete event by event information of the charge partitions. If we can reasonably estimate the relation between the charge $Z_{i}$ and the mass number $A_{i}$, and the value of the freeze out volume $V_{F O}$ (see Sect. 2.3, Chap. 2), we can then obtain the event by event Q-value and Coulomb energy according to the equation

$$
E_{I}=\sum_{k=1}^{M} m_{i}+E_{\text {coul }}^{V_{\mathrm{FO}}}\left(Z_{i}, i=1, \ldots M\right)
$$

where $M$ is the total multiplicity and $m_{i}$ are the mass excess of the primary products. Equation (65) represents the interaction energy at the time of fragment formation, while $E_{k}=E^{*}-E_{I}$ is the non interacting part. $E^{*}$ is related to the total deposited energy, which is also measured event by event by calorimetry (see Sect. 2.4, Chap. 2). The interaction energy fluctuation can then be studied 
as a function of total energy, the heat capacity can be measured according to equation (56),

$$
C=\frac{C_{1}^{2}}{C_{1}-\frac{\sigma_{I}^{2}}{T^{2}}},
$$

and abnormal fluctuations can be looked for.

To illustrate this method [47], equation (66) is applied in Figure 32 to two well-known and sophisticated models, often used to simulate heavy ion reaction data, the SMM [48] and GEMINI [49] models. SMM modelizes multifragmentation at low freeze out density and for the typical volumes used (around three times the normal source volume) shows a first order liquid-gas like phase transition [50,51]. GEMINI describes fragment production as a sequence of binary fission-like emissions at a density close to the normal nuclear matter density. An explicit study of the thermodynamics of this model has never been done. Because of the hypothesis of low emission rate (i.e. low vapour-like pressure) and high

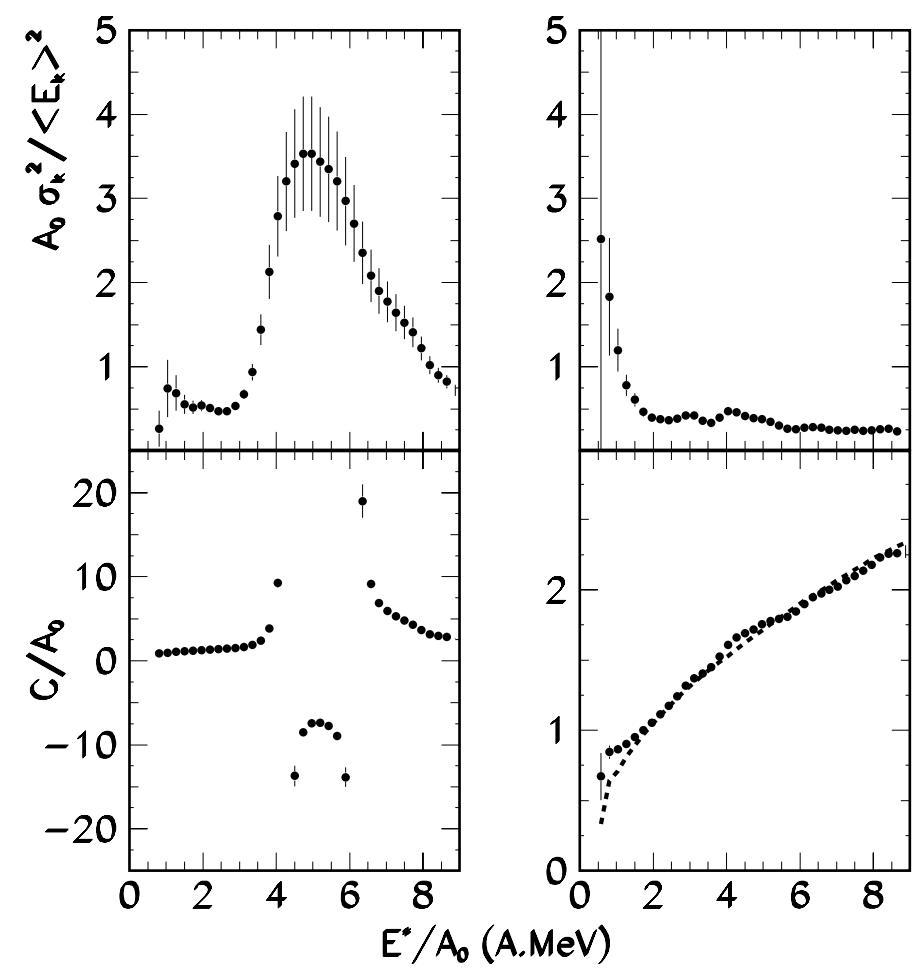

Figure 32. Reconstructed normalized kinetic energy fluctuations at freeze out (upper part) and the corresponding heat capacity (lower part) for SMM (left) and GEMINI (right) simulations as a function of the excitation energy. Dashed line: numerical derivative of the theoretical caloric curve. 
density, we however expect that the model should be close for all excitation energies to the coexistence line on the liquid border, and it should not show negative heat capacity.

In both cases the temperature estimated from the average value of the non interacting part of the total energy $\left\langle E_{k}\right\rangle=E_{\text {tot }}-\left\langle E_{I}\right\rangle$ according to equation (55) (see Sect. 2, Chap. 2 for details) is in good agreement with the input temperature of the models. The heat capacity is estimated from equation (66). As we can see from Figure 32, abnormal fluctuations are obtained for the SMM simulation while the heat capacity is a monotonically increasing function of the excitation energy in the GEMINI case, and very close to the numerical derivative of the theoretical caloric curve. This result indicates that equation (66) is a powerful tool to extract heat capacities also for realistic nuclear models, for sequential as well as simultaneous emission processes, the only condition being the thermal equilibrium of the data sample.

The central question of equilibrium will be discussed in the next chapter.

\section{Equilibria out of equilibrium}

An important conceptual problem linked with multifragmentation experiments, is that the outcomes of a nuclear collision are not confined in an external container but dynamically de-excite in the vacuum. The most sophisticated nuclear transport models predict that fragment formation is a highly dynamical process, with comparable time scales for the formation and the break-up of the intermediate excited state, which lifetime does not overcome a few tens of $\mathrm{fm} / \mathrm{c}$. In the absence of boundary conditions, collective flows induced by a memory of the entrance channel (transparency) or by the dynamics of the compression stage (radial flow) can be present at the fragmentation time.

Approximate models [48] simply subtract the energy stored in the collective motion from the global energetics, but because of the short lifetime and this time odd component, one may doubt about the applicability of equilibrium concepts.

In this chapter we will show that information theory allows a thermodynamically consistent description of open short-lived finite systems in evolution under a collective flow. We will apply this formalism to the lattice gas Model for the particular cases of a memory of the entrance channel (Sect. 1.3) and radial expansion (Sect. 1.4). In both cases the properties of the system appear to be affected in a sizeable way when flow dominates the global energetics.

\subsection{What is equilibrium? [52]}

The whole discussion of this work - and more generally, the pertinence of equilibrium statistical mechanics to actual physical problems - is based on the validity of the fundamental postulate discussed in Section 1.2 (Chap. 1). Concerning macroscopic systems, the validity of equilibrium is somewhat an academic question: an infinite system can always be divided in an infinite number of infinite subsystems, 
and there is no reason why the subsystems would differ in their microscopic structure from the global system. If the system is small, the same method cannot be applied: the partitioning into subsystems leads to qualitatively different objects, because the interfaces cannot be neglected in the global energetics. This is why the traditional approach to the concept of equilibrium in small systems is given by the ergodic theorem: if the system is ergodic (i.e. during its dynamical evolution it explores the phase space according to its density of states) then the time average of any observable $A$ will be asymptotically equal to the ensemble average

$$
\lim _{T \rightarrow \infty} \frac{1}{T} \int_{0}^{T} d t A(t)=\frac{1}{W_{\text {tot }}} \int d A A W(A) .
$$

The statistical ensemble is then given by the collection of different snapshots of the system evolving in time, provided that the observation time is much longer than a typical equilibration time, i.e. the time needed to explore the whole accessible phase space.

This approach is not satisfactory for a number of reasons. First, its domain of applicability is extremely limited: the only possible state variables are the variables conserved by the dynamics and no equilibrium is possible if the controlled variables are intensive (for instance the canonical ensemble is not defined). Moreover the ergodicity of the Hamiltonian is irrelevant if the system is not confined in a box, and even for truly ergodic systems the characteristic time $T$ is of the order of the number of accessible states $T \approx W=\exp S=\exp (s N)$ i.e. exponentially increasing with the number of particles: equilibration is almost impossible to attain for complex systems. Last but not least, in experiments on a microscopic scale all observations lead to the collapse of the wave packet: observables are never measured as a function of time but we rather have a huge set of instantaneous observations of similarly prepared systems.

However, ergodicity is not the unique way to produce an equilibrium, and in fact the equilibrium concept does not involve the way the statistical ensemble is produced but only the probability of all the different realizations. Indeed, as we have seen in Section 1 (Chap. 1), an equilibrium corresponds to any ensemble of states that maximizes the entropy in a given space under the constraint of (a number of) observables known in average [4]. If the nuclear dynamics is sufficiently sensitive to the initial conditions, the ensemble of outcomes of similarly prepared nuclear collisions can be considered as a statistical ensemble for which the important observables are controlled by the dynamics and by the event sorting performed on specific observables. (For a rigorous discussion on the mixing property that the Hamiltonian must have for the dynamics to be sufficiently sensitive to the initial conditions, see reference [52]. Here it is sufficient to recall that mixing is almost always verified in model Hamiltonians of complex short ranged systems.)

In this information theory approach, the state variables are determined by the dynamics and are not restricted to time even observables, but can also be time odd quantities such as transparency or radial flow. 
It is important to stress that this statistical mechanics viewpoint, where we assume that the whole (limited) information we have on a system is also the only relevant one (see Sect. 1.2, Chap. 1), is the least ambitious and the most economic approach to many body physics. This means that equilibrium has never to be proved: it is impossible to demonstrate that the only information contained in a set of data is given by the measured state variables until all the virtually infinite number of possible observables has been measured. Rather a deviation from a given equilibrium has to be found in order to demonstrate the relevance of a specific dynamic effect: this deviation constitutes an extra constraint that can be put on the statistical ensemble, and a statistical treatment is still worthwhile as long as the number of dynamical constraints is small respect to the relevant degrees of freedom.

In this sense a statistical treatment of data has to be interpreted as a reduction of the complex and redundant many body information to the relevant collective degrees of freedom that are associated to the transport properties of nuclear matter, and that can be theoretically accessed through dynamical transport calculations.

\subsection{Negative heat capacity and the freeze-out volume}

As a first example, let us consider the standard freeze out hypothesis where the configurations are fixed when the nuclear interaction among prefragments becomes negligible. This requirement corresponds to an average distance between prefragment surfaces. Then the ensemble of events is characterized by several variables such as the energy and the spatial extension $R^{2}$ or $R^{3}$ which is fluctuating event by event. This can be accounted for by considering the ensemble average $\left\langle R^{3}\right\rangle$ as a state variable and introducing a Lagrange multiplier $\lambda_{V}$ closely related to a pressure [4].

We have already discussed in Sections 6.4 and 7.3 (Chap. 1) that bimodalities and negative heat capacities for the liquid gas phase transition are connected to volume fluctuations. Indeed density is the order parameter of the transition, which means that if the number of particles is fixed, the heat capacity can only be negative in the isobar ensemble. Since the freeze out of a system without boundary conditions is represented by a fluctuating volume (isobar) ensemble, this means that negative heat capacity has to be expected in multifragmentation if this phenomenon takes place at low density.

It is interesting to remark that the absence of boundary condition (i.e. a confining box of infinite volume) is a physical situation which belongs to the isobar ensemble. Indeed in the infinite volume limit the isobar partition sum with $p=0$ is identical to the partition sum of a system confined in a box

$$
\lim _{V_{b o x} \rightarrow \infty} Z\left(E, V_{b o x}\right)=\lim _{V_{b o x} \rightarrow \infty} \int d V \Theta\left(V_{b o x}-V\right) W(E, V)=\int d V W(E, V) e^{-\lambda_{V} V}
$$

where $V=R^{3}$ and $\lambda_{V}=0$. The equivalence of the two ensembles in the infinite volume limit is depicted in Figure 33, that shows the caloric curve and partial 


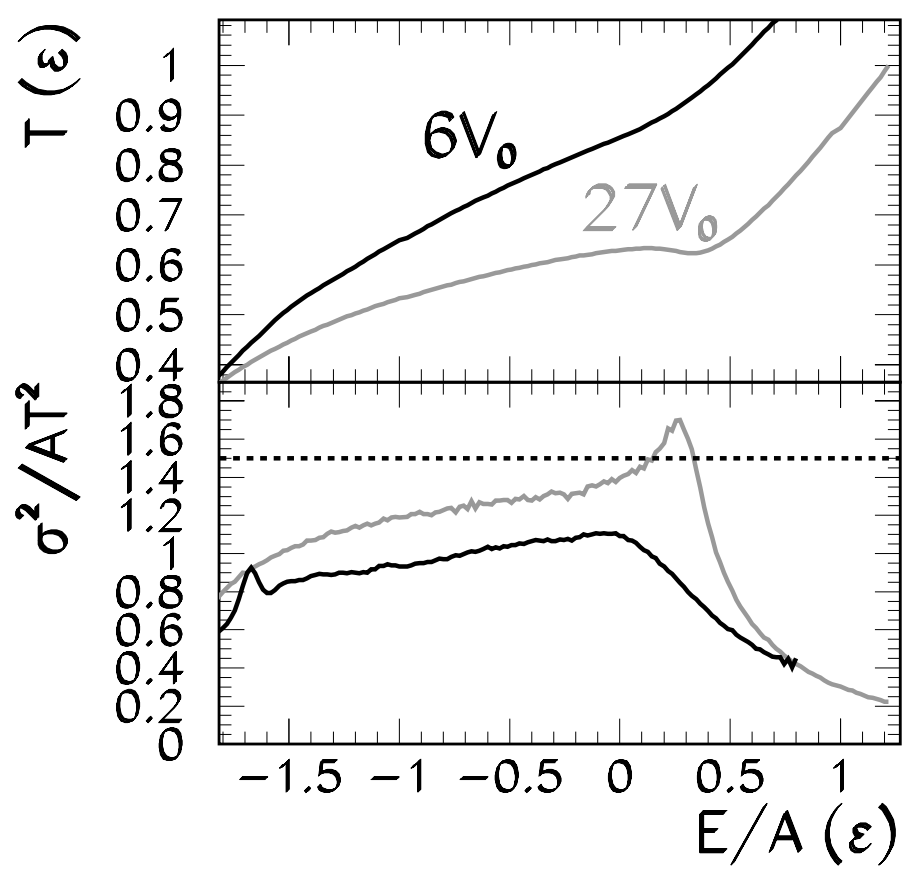

Figure 33. Caloric curves (upper part) and normalized partial energy fluctuations (lower part) for a system of 216 particles in the isochore lattice gas model for two different lattice sizes.

energy fluctuation in the isochore Lattice Gas model with a linear dimension $L=11$ and $L=18$ : in the big volume case the caloric curve backbends in the coexistence zone and the fluctuations overcome the canonical limit (see Eq. (66)) while in the $L=11$ case energy fluctuations are suppressed by the constraint of the box because of the correlation between energy and volume (see Fig. 23). The same result has been reported in the framework of the IMFM model in reference [53]. The curvature anomaly in the isochore system appears because the number of particles is too small for the boundary conditions to be effective: in the true thermodynamic limit $V \rightarrow \infty, N \rightarrow \infty, N / V=$ cte the anomaly disappears in agreement with the Van Hove theorem [19].

\subsection{The incomplete momentum relaxation ensemble}

Let us now consider a symmetric head-on collision with a too short reaction time to fully relax the incoming momentum. This situation seems to be verified at relativistic energies [54]. It corresponds to the observation of an additional one body state variable, the memory of the initial momenta $\left\langle\tau p_{z}\right\rangle$, where $p_{z}$ is the momentum along the beam axis and $\tau=-1(+1)$ for the particles initially belonging to the target (projectile). Let us assume that the total energy $E$ is also 
known only in average. The maximization of the entropy leads to the partition sum of the incomplete momentum relaxation ensemble (IMRE)

$$
Z_{\beta, \alpha}=\sum_{n} \exp \left(-\beta E^{(n)}+\alpha \sum_{i=1}^{A} \tau_{i} p_{i z}^{(n)}\right),
$$

where the index $i$ stands for the $i$ th particle while $(n)$ counts the events. $\alpha$ and $\beta$ are Lagrange multipliers associated to the constraint of the incomplete stopping $\left\langle\tau p_{z}\right\rangle$ and of the total center of mass energy $\langle E\rangle$. The relative probability of an event $(n)$ results

$$
\begin{aligned}
p^{(n)} & \propto \exp \left(-\beta E^{(n)}+\alpha \sum_{i=1}^{A} \tau_{i} p_{i z}^{(n)}\right) \\
& \propto \exp \left[-\beta\left(\sum_{i=1}^{A} \frac{\left(\vec{p}_{i}^{(n)}+\tau_{i} \vec{p}_{0}\right)^{2}}{2 m}+\sum_{i j}^{A} U_{i j}\right)\right]
\end{aligned}
$$

where $U_{i j}$ is the two body interaction and we have introduced $\vec{p}_{0}=m \alpha / \beta \overrightarrow{u_{z}}$. The average kinetic energy per particle is given by a thermal component $\left\langle e_{t h}\right\rangle=3 /(2 \beta)$ plus a flow $\left\langle e_{f l}\right\rangle=p_{0}^{2} /(2 m)$ while the equation of states related to $\alpha$ leads to $\left\langle\tau p_{z}\right\rangle=A p_{0}$. The degree of transparency can be measured from the ratio $\left\langle e_{f l}\right\rangle /\left\langle e_{t h}\right\rangle$ which is nothing but the quadrupolar deformation in momentum space

$$
\Delta p^{2} / p^{2}=\left(\left\langle p_{z}^{2}\right\rangle-\left\langle p_{x}^{2}\right\rangle\right) / 3\left\langle p_{x}^{2}\right\rangle
$$

In the limit $p_{0}=0$ the IMRE reduces to the usual canonical ensemble, while in the general case it can be interpreted as two thermalized sources with a non zero relative velocity $2 \vec{p}_{0} / m$ along the beam axis $\vec{u}_{z}$.

This theory can be extended to take into account other observables. If for example the average momentum dispersion along the beam axis is known, an extra term $\eta p_{i z}^{(n) 2}$ can be added in equation (67) to control via the Lagrange multiplier $\eta$ the collective flow fluctuation, while a term $\lambda R^{(n) 2}$ can be used to impose an average freeze out volume.

In actual heavy ion experiments the centrality selection criteria imply a sorting of data according to the total deposited energy [47], or to variables which are strongly correlated to it. This means that a (strict or loose) total energy conservation has to be implemented to equation (68). In this case the equations of state are not analytical but can still be numerically evaluated.

To understand the effect of transparency on the evaluation of thermodynamic quantities, calculations in the IMRE equation (67) are performed with the lattice gas Hamiltonian for a system of $A=216$ particles at a subcritical pressure $\lambda=$ $3.3 \times 10^{-4}[14,15]$ and clusters are defined within the standard Coniglio-Klein prescription [30]. For simplicity in the following calculations the longitudinal momentum dispersion has been kept fixed, $(\beta / 2 m+\eta)^{-1}=0.04 \epsilon$. Since in this 


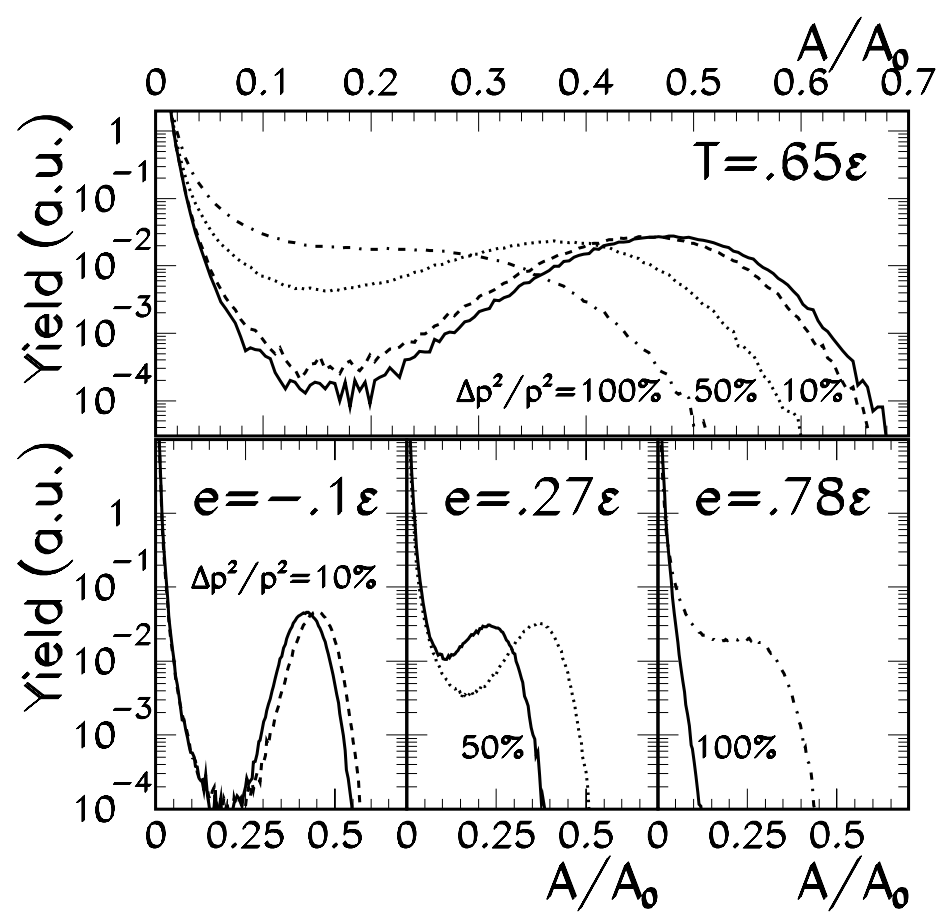

Figure 34. Fragment size distributions in the lattice gas model. Upper (lower) part: canonical (microcanonical) calculations. Full lines: isotropic momentum distributions. Dashed, dotted and dashed-dotted lines: different degrees of transparency (see text).

classical approach the configurational and kinetic partition sums are factorized, for a given $\beta$ the lattice configurations will be independent of the transparency. However the active bond probability will explicitly depend on $p_{0}$ meaning that cluster observables can be affected by the incomplete relaxation. The upper part of Figure 34 shows cluster size distributions at a temperature $\beta^{-1}=0.65 \epsilon$ which corresponds to the transition temperature in the canonical ensemble, and different degrees of transparency.

A quantitative comparison with experimental data would require to fix the Lagrange parameters $\lambda, \beta, p_{0}$ from each specific set of data, and to include symmetry as well as Coulomb terms in the Hamiltonian, however from this illustrative example we can clearly see that partitions are affected by a collective longitudinal component and a higher degree of fragmentation does not necessarily imply higher temperatures but can also be consistent with an increased degree of transparency of the collision. To quantify this statement, the lower part of Figure 34 compares the clusters size distributions of the microcanonical IMRE for different $\Delta p^{2} / p^{2}$ with the standard microcanonical ensemble (fixed energy, spherical momentum distribution) at the same total energy. It is clear that thermal agitation 


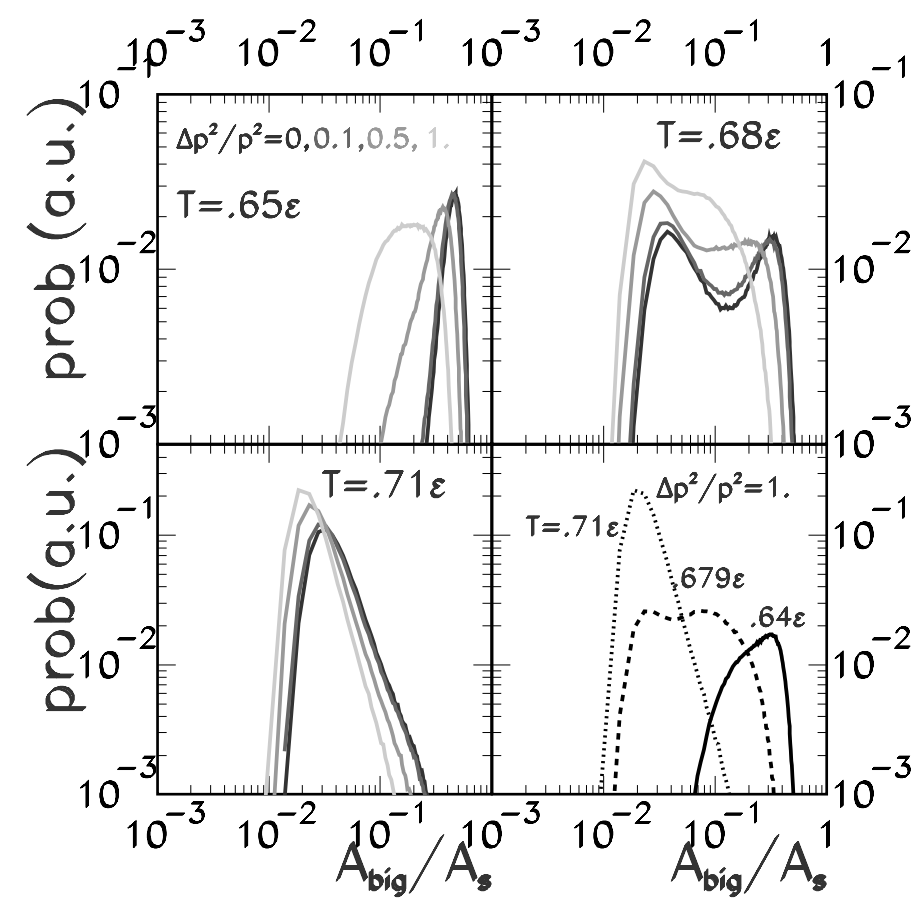

Figure 35. Distribution of the biggest fragment in the coexistence region of the isobar lattice gas model for different degrees of transparency.

is much more effective than transparency to break up the system: at $e=0.78 \epsilon$ where the standard microcanonical ensemble predicts a complete vaporization of the system, a residue persists if the non relaxed momentum component is as large as the relaxed one $\left(\Delta p^{2} / p^{2}=100 \%\right)$. This is in qualitative agreement with the trend observed in central collisions at relativistic energies [55]. However up to $10 \%$ transparency the distributions remain unchanged, meaning that when the velocity difference between the quasi-projectile and the quasi-target is of this order, the debate on equilibrium based on the number of emission sources [56] or on their deformation in momentum space is an academic question.

The effect on the order parameter $Z_{\text {big }}$ distribution is shown in Figure 35. The bimodality signal (see Sect. 6, Chap. 1) appears very robust respect to an incomplete relaxation of the incoming momentum. Up to about $50 \%$ transparency the transition temperature is almost independent of the longitudinal flow: the extra energy stocked in the longitudinal motion can be simply approximately subtracted from the total energy balance. This finding is in good agreement with experimental data (see Sect. 3, Chap. 2).

Note on passing that because of finite size effects the different order parameters do not point exactly to the same value for the transition temperature: at the temperature $T_{E}=0.65 \epsilon$ at which the two energy peaks have the same height, 
the size distribution is still dominated by a percolative clusters; conversely at the transition temperature $T_{A_{\max }}=0.68 \epsilon$ defined by the $A_{\max }$ order parameter the system is already in its gas phase from the energetic point of view. This finding is also in qualitative agreement with experimental data (see Sect. 2, Chap. 2) and shows that the uncertainty on the transition temperature due to transparency effects is less important than the intrinsic finite size uncertainty due to the choice of the order parameter.

Let us now analyze the possible bias induced by transparency on the experimental methods used to determine the heat capacity [47]. As we have already mentioned (and we will see in further detail in Sect. 2, Chap. 2), the role of the potential energy is played in multifragmentation data by the $Q$-values of the measured partitions. The $Q$-value can be computed in the lattice gas model in a liquid drop approximation as

$$
Q^{(n)}=\sum_{i=1}^{M^{(n)}} a_{v} A_{i}+a_{S} A_{i}^{2 / 3}
$$

where $M^{(n)}$ is the multiplicity of the $n$th event and $a_{v}, a_{s}$ are the volume and surface energy coefficients. The average kinetic energy and the average $Q$-value obtained with equation (69) are shown in the left part of Figure 36 for the microcanonical ensemble, and for a system with the same total deposited energy and an increasing degree of transparency $\Delta p^{2} / p^{2}$. The energy range explored corresponds to the phase transition region. The increased probability of heavy clusters with an increasing degree of transparency shown in Figure 1 induces an overestimation of the average kinetic energy, hence of the estimated temperature. The peak in the partial energy fluctuation (right part of Fig. 36) which signals the phase transition [45] disappears in this extreme scenario of an increase of energy going entirely into the relative motion of the two sources. Moreover the normalized fluctuations are systematically lower than in the microcanonical ensemble where
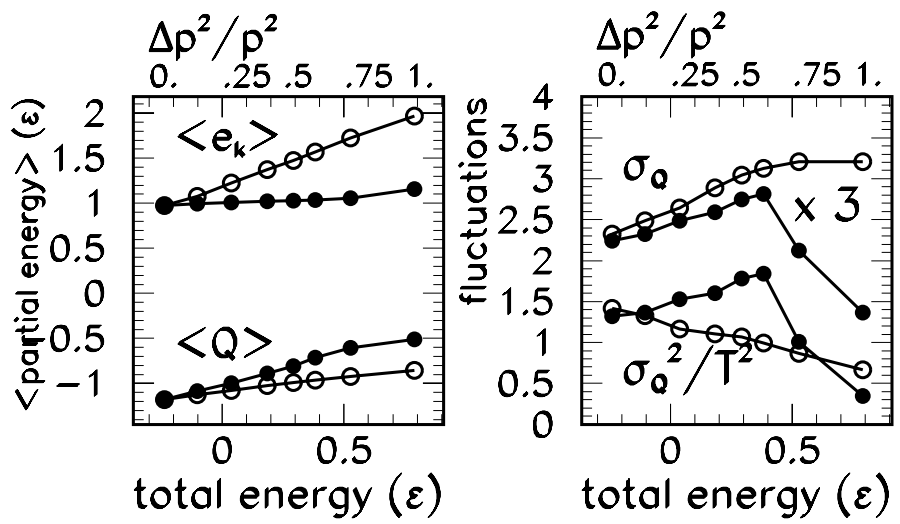

Figure 36. Average partial energies (left) and variances (right) in the microcanonical ensemble (full circles) and in the IMRE (open circles) at fixed energy. 
the incoming momentum is fully relaxed. Since a negative heat capacity corresponds to abnormally high partial energy fluctuations (see Sect. 7.1, Chap. 1), this implies that an incomplete relaxation of the incoming momentum can prevent the observation of negative heat capacity.

\subsection{Equilibrium under radial flow}

Another important form of collective motion in heavy ion collisions is radial flow which starts to be observed in central collisions around $30 \mathrm{~A} \mathrm{MeV}$ incident energy [56] and becomes the dominant fraction of the detected energy in the relativistic domain [55]. We can describe this dynamical situation as an equilibrium with non random directions for velocities, which are preferentially oriented in the radial direction. In the canonical formulation this corresponds to the independent observation of the average energy $\langle E\rangle$ and the average local radial momentum $\left\langle p_{r}(r)\right\rangle$. The probability of a microstate $(n)$ reads

$$
p^{(n)} \propto \exp \left(-\beta E^{(n)}-\sum_{i=1}^{A} \gamma\left(r_{i}\right) \vec{p}_{i} \cdot \vec{u}_{r_{i}}\right),
$$

where $r_{i}$ is the position of the $i$ th particle, $\beta$ the inverse canonical temperature and $\gamma(r)$ the local Lagrange multiplier. Imposing in the local equation of state $\left\langle p_{r}\right\rangle=\partial \log Z / \partial \gamma$ that the observed velocity is self-similar $\left\langle p_{r}(r)\right\rangle=m \alpha r$ we obtain $\gamma(r)=-\beta \alpha r$ which gives for the argument of the exponential in the probability (70)

$$
-\beta \sum_{i=1}^{A} \frac{\left(\vec{p}_{i}-\vec{p}_{0}\left(r_{i}\right)\right)^{2}}{2 m}+\beta \frac{\alpha^{2} m}{2} \sum_{i=1}^{A} r_{i}^{2}-\beta \sum_{i j}^{A} U_{i j},
$$

with the local radial momentum $\vec{p}_{0}(r)=m \alpha r \vec{u}_{r}$. In the expanding ensemble the total average kinetic energy is the sum of the thermal energy $\left\langle e_{t h}\right\rangle=3 /(2 \beta)$ and the radial flow $\left\langle e_{f l}\right\rangle=m \alpha^{2} / 2\left\langle R^{2}\right\rangle$. The situation is equivalent to a standard Gibbs equilibrium in the local expanding frame. This scenario is often invoked in the literature [48] to justify the treatment of flow as a collective radial velocity superimposed on thermal motion; however equation (71) contains also an additional term $\propto r^{2}$ which corresponds to an outgoing pressure. The probability under flow being the exponential of equation (71), it diverges at infinity reflecting the trivial dynamical fact that asymptotically particles flow away. This divergence should be cured by introducing an external confining pressure which is not a mathematical artifact, but has to be interpreted as discussed in Section 1.1 (Chap. 2) above as a Lagrange multiplier imposing a finite freeze out volume. Equation (71) has then to be augmented by a term $-\lambda \sum_{i} r_{i}^{2}$ with $\lambda \geq m \alpha^{2} / 2 T$ leading to a positive pressure coefficient $\lambda_{\text {eff }}=\lambda-m \beta \alpha^{2} / 2$.

This discussion has an important conceptual consequence: an equilibrium under flow in the isochore ensemble is meaningless and the common practice adopted in nuclear physics statistical models $[48,60]$, of adding up a radial momentum to fragment partitions equilibrated in a box, is theoretically inconsistent. 
Even more, the experimental observation of a radial flow [56] demonstrates that the isobar ensemble is the only relevant ensemble to nuclear fragmentation.

It is interesting to notice that the effective pressure can never be negative: for a given amount of flow at each temperature equilibrium can exist only for pressures exceeding a limiting value $\lambda \geq m \alpha^{2} / 2 T$. At lower pressures the information theory approach becomes meaningless and the problem can only be addressed through a dynamical approach.

The effective pressure $\lambda_{\text {eff }}$ as well as the associated average volume (normalized to the ground state volume $V_{0}=A$ ) are shown in the upper left part of Figure 37 as a function of the collective radial flow for a given pressure and temperature, $\lambda=1.23 \times 10^{-2}, \beta^{-1}=0.65 \epsilon$. The Lagrange parameter $\lambda_{\text {eff }}$ being a decreasing function of $\alpha$, the flow reduces the effective pressure, so that the critical point is moved towards higher pressures in the presence of flow [57]. However one can see that the effect is very small up to $m \alpha \approx 0.6$ (which corresponds to $\Delta p^{2} / p^{2}=\left\langle e_{f l}\right\rangle /\left\langle e_{t h}\right\rangle \approx 67 \%$ contribution of flow to the total kinetic energy). In this regime the cluster size distributions displayed in the upper right part of Figure 37 are only slightly affected. On the other side if the collective flow overcomes a

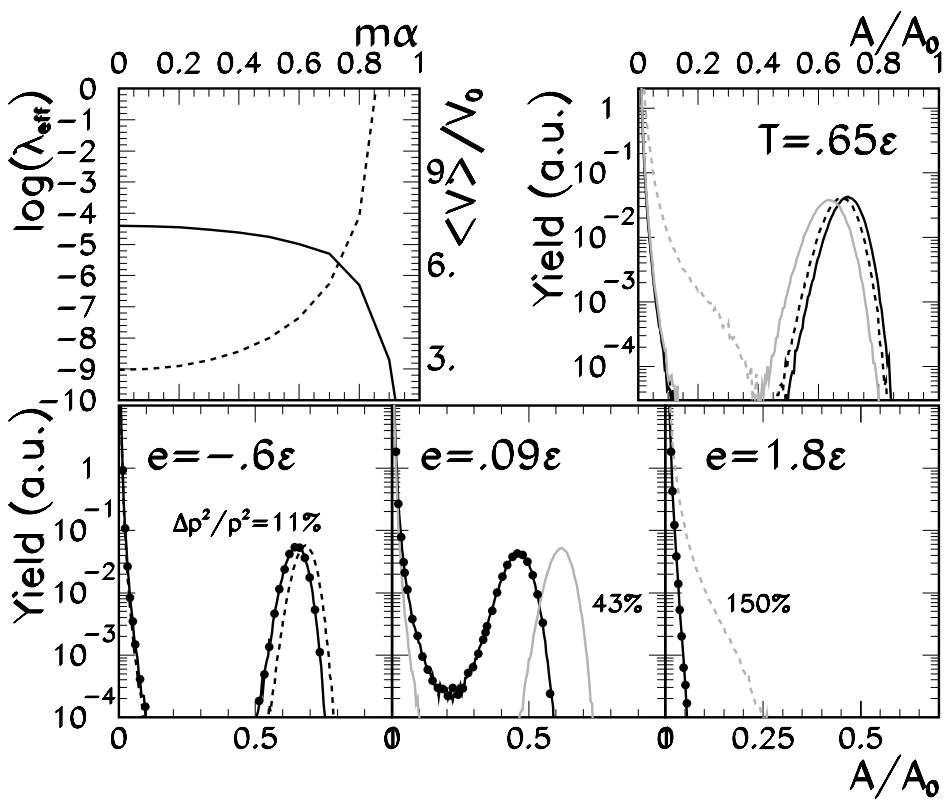

Figure 37. Upper left: effective pressure (full line) and average volume (dashed line) as a function of the collective radial flow (see text). Upper right and lower part: fragment size distributions in the expanding lattice gas model. Distributions without flow (full black lines) are compared with distributions with 11\% (dashed black), 43\% (full grey) and 150\% (dashed grey) contribution of radial flow at the same temperature (upper right) and at the same total energy (lower part). Symbols: calculations with flow at the same thermal energy and average volume as the full black lines. 
threshold value $\Delta p^{2} / p^{2} \approx 100 \%$ the average volume (dashed line in the upper part of Fig. 37) shows an exponential increase and the outgoing flow pressure leads to a complete fragmentation of the system (dashed grey line in the lower part of Fig. 37). Again an oriented motion is systematically less effective than a random one to break up the system. This is shown in the lower part of Figure 37 which compares for a given $\lambda$, distributions with and without radial flow at the same total deposited energy: for any value of radial flow equilibrium in the standard microcanonical ensemble corresponds to more fragmented configurations.

Concerning heavy ion collisions, it is important to stress that the pressure $\lambda$ as well as the other state variables are consequences of the dynamics. They cannot be accessed by a statistical treatment but have to be extracted from simulations and/or directly inferred from data itself [47]. Different models assume that fragmentation occurs in an average freeze out volume which may depend on the thermal energy but does not depend on flow $[48,58,59]$. This is true if the system fragments at the turning point of its expansion $(\alpha=0)$ [58] or when the interaction between fragment surfaces becomes negligible [48] or more generally is insufficient to modify the $\mathrm{N}$-body correlations [59]. In this case the presence of flow does not affect the configuration space and can only modify the partitions because of the bonds breaking taken into account by the Coniglio-Klein algorithm. However this effect is negligible as already observed by Das Gupta et al. [60] and shown in the lower part of Figure 37. In this figure the symbols represent the size distributions with collective flow at the same thermal energy and average volume as the standard microcanonical results (full lines). Even for the largest amount of flow considered the two distributions are identical, meaning that in this hypothesis all thermodynamic analysis of fragmentation data stay valid in the presence of even strong collective flows [60].

\section{Heat capacity measurements}

In this chapter we will concentrate on the heat capacity measurements performed on quasi-projectile events from peripheral $35 \mathrm{~A} \mathrm{MeV} \mathrm{Au+Au} \mathrm{collisions} \mathrm{and} \mathrm{central}$ events from $25 \mathrm{~A} \mathrm{MeV} \mathrm{Au}+\mathrm{C}, 25$ and $35 \mathrm{~A} \mathrm{MeV} \mathrm{Au}+\mathrm{Cu}$, and $35 \mathrm{~A} \mathrm{MeV} \mathrm{Au}+\mathrm{Au}$ collisions $[47,61]$.

All these measurements have been performed at the K1200-NSCL Cyclotron of the Michigan State University with the MULTICS-Miniball apparatus (for experimental details, see Ref. [47]).

Results consistent with the ones presented here are obtained with central collisions INDRA data [47,62] and ISIS data [63].

\subsection{Probing equilibrium}

In order to perform the fluctuation analysis (see Sect. 7.1, Chap. 1), one has to collect a data sample which corresponds to a (collection of) microcanonical ensembles. The microcanonical ensemble is relevant for the analysis of experimental 
data because of the absence of a heat bath, and since using calorimetry techniques the excitation energy can be measured on an event-by-event basis; for any arbitrary shape of the excitation energy distribution the events can thus be sorted in constant energy bins, i.e. in microcanonical ensembles.

Single source complete events have been selected with a constant value for the collected charge in each energy bin $[47,61]$. In the case of the Au quasi-projectile, a possible contribution of mid-rapidity emission is minimized by substituting the backward light particle emission by the symmetric of the forward emission in the quasi-projectile reference frame [47]. The observed event isotropy in momentum space [47] indicates that the directed flow component coming from a memory of the entrance channel (see Sect. 1.2, Chap. 2) is negligible. A detailed analysis of the average kinetic energy of the fragments in comparison with the statistical model SMM [48] indicates the presence of a radial collective flow ranging from $0 \mathrm{AMeV}$ for the most peripheral, to at most $1 \mathrm{AMeV}$ for the central collisions of the $\mathrm{Au}+\mathrm{Au}$ system. This collective contribution to the excitation energy has been removed in the subsequent analysis, but we should keep in mind that the determination of radial flow from the kinetic energy spectra is not unambiguous, since the effect of a radial flow can be mocked up by a smaller freeze out volume, because of the increased Coulomb repulsion. We will come back to this point in the next section.

According to the discussion of Section 1.1 (Chap. 2), an equilibrium is a minimum information data sample, i.e. a sample which is completely characterized by the collective state variables. This means that in equilibrium the fragmentation pattern should be determined by the size, charge, energy and average freeze out volume solely, independent of the way the source has been formed, i.e. of the entrance channel of the reaction. A number of different impact parameters, leading to a widely spread excitation function, is available for the quasi-projectile data, that can therefore be compared to central events in different excitation energy bins. The comparison is presented in Figure 39 for the biggest fragment produced in every event and in Figure 38 for the other fragments. Fission has been recognized and reconstructed through a velocity correlation technique [61,64]. The remarkable scaling between central and peripheral collisions means that these data samples can be analyzed, at least in a good first order approximation, within the statistical microcanonical methods [56].

\subsection{Rough data}

In order to extract the heat capacity from multifragmentation data, the interaction energy can be evaluated as in equation (65)

$$
E_{I}=\sum_{k=1}^{M} m_{i}+E_{\text {coul }}^{V_{\mathrm{FO}}}\left(Z_{i}, i=1, \ldots, M\right)
$$



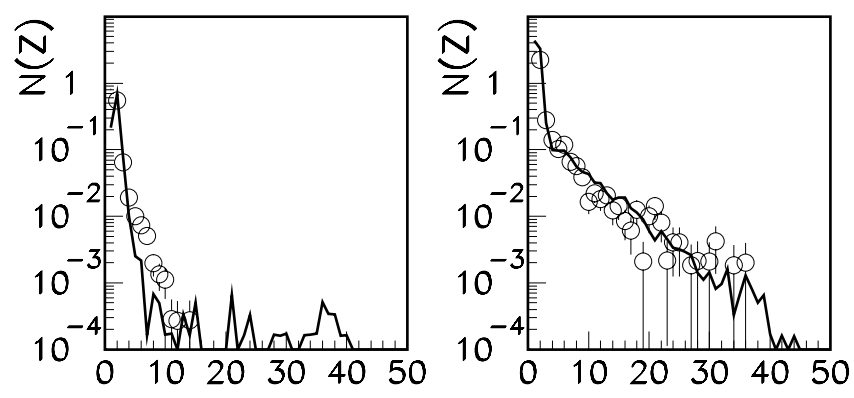

Z

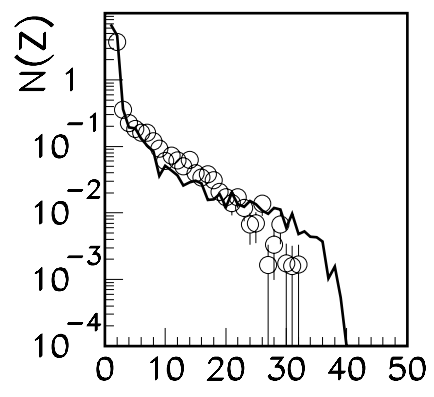

Z

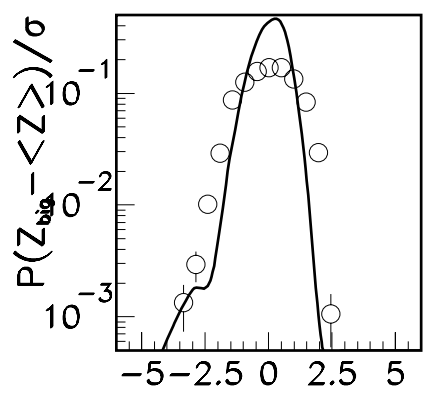

$\left(Z_{\text {big }}-<Z>\right) / \sigma$

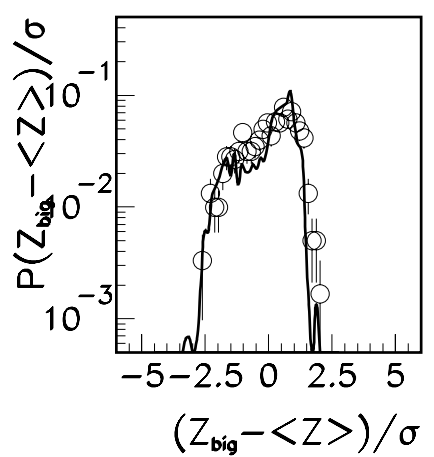

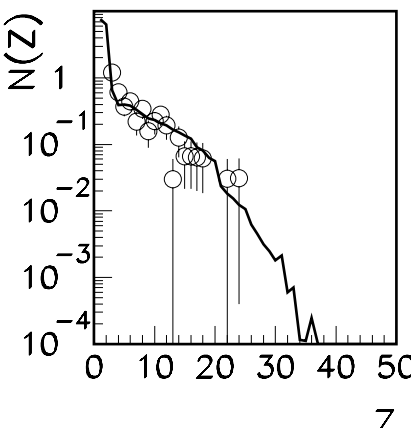

Figure $38 . \quad$ Symbols: size distributions of all detected fragments but the biggest, for central collisions $(\mathrm{Au}+\mathrm{C}$ at $25 \mathrm{AMeV}, \mathrm{Au}+\mathrm{Cu}$ at 25 and $35 \mathrm{AMeV}, \mathrm{Au}+\mathrm{Au}$ at $35 \mathrm{~A} \mathrm{MeV})$. Lines: QP from $\mathrm{Au}+\mathrm{Au}$ peripheral collisions at $35 \mathrm{AMeV}$ in the same calorimetric energy bins as the central collisions.
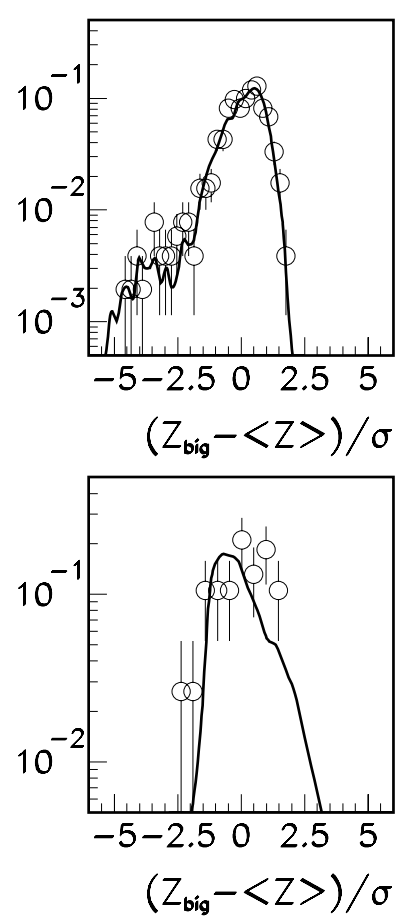

Figure 39. Symbols: size distributions of the biggest detected fragment in every event, for central collisions $(\mathrm{Au}+\mathrm{C}$ at $25 \mathrm{AMeV}, \mathrm{Au}+\mathrm{Cu}$ at 25 and $35 \mathrm{AMeV}, \mathrm{Au}+\mathrm{Au}$ at $35 \mathrm{~A} \mathrm{MeV}$ ). Lines: QP from $\mathrm{Au}+\mathrm{Au}$ peripheral collisions at $35 \mathrm{~A} \mathrm{MeV}$ in the same calorimetric energy bins as the central collisions. 

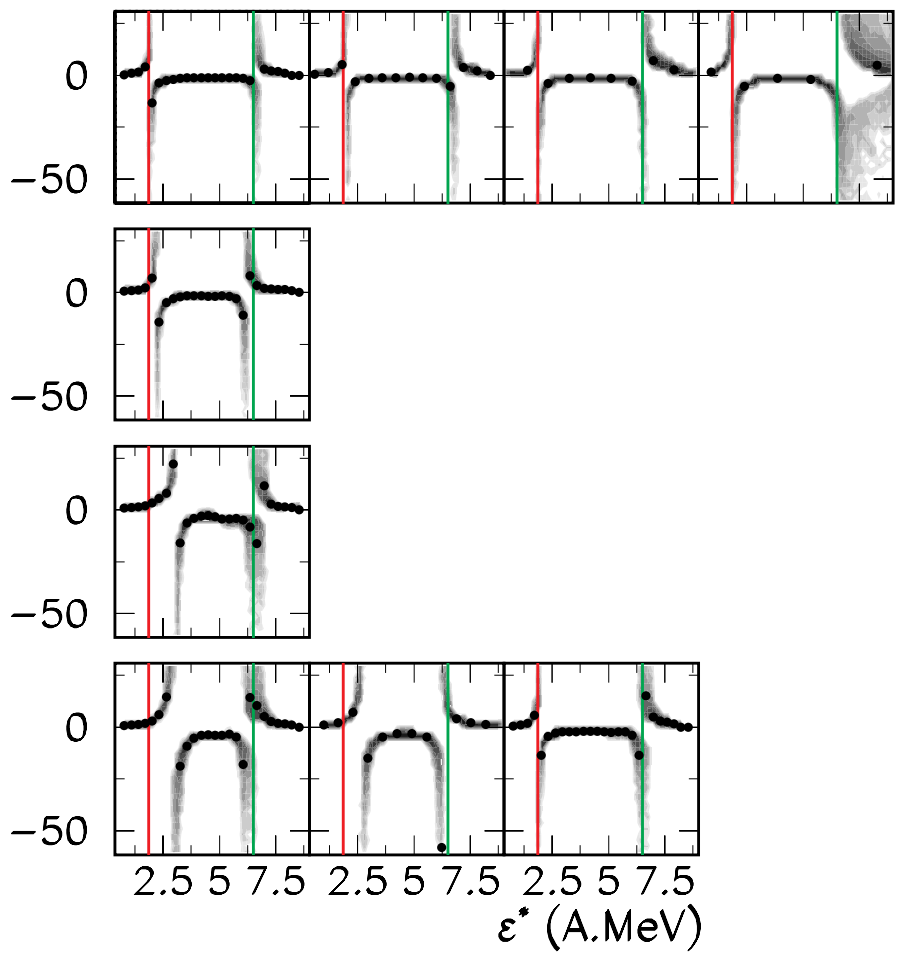

Figure 40. Experimental heat capacity per nucleon for QP data without correction for the side feeding. Fission events are excluded. From top to bottom: freeze out volume varying from $\approx 3 V_{0}$ to $6 V_{0}$. Upper row, from left to right: bin energy width of $0.3,0.6,0.91 .5 \mathrm{~A} \mathrm{MeV}$. Lower panel, second picture: flat excitation energy distribution; third picture: $a=8$. For the other pictures the level density parameter is a function of the fragment mass varying from 8 to 12 according to reference [48].

while an estimator of the microcanonical temperature $T$ can be obtained by inverting the kinetic equation of state as given by equation (55)

$$
\begin{aligned}
\left\langle E_{k}\right\rangle & =E^{*}-\left\langle E_{I}\right\rangle \\
& =\left\langle\sum_{i=1}^{M} \frac{A_{i}}{a_{i}(T)}\right\rangle T^{2}+\left\langle\frac{3}{2}(M-1)\right\rangle T,
\end{aligned}
$$

where the brackets $\langle\cdot\rangle$ indicate the average on the events with the same $E^{*}$. The mass excess $m_{i}$ are deduced from the measured charges $Z_{i}$ by assuming that all measured fragments share the same $N / Z$ ratio of the source. Then the only unknown parameters are the freeze out volume $V_{F O}$ and the level density parameter $a_{i}$. The resulting heat capacity is displayed in Figure 40. A clear negative heat capacity region is present for all volumes smaller than about 11 times the normal source volume and all values of $a$ compatible with the experimental 
systematics [65]. If this result indicates a great robustness of the negative heat capacity measurement, it is also important to stress that no quantitative thermodynamics can be extracted from Figure 40. Indeed the two key parameters $V_{F O}$ and $a$ are still model dependent and side feeding corrections should be taken into account. These points will be discussed in the next section. Moreover the inefficiencies of the experimental apparatus can distort in an important way the measured signal. These distortions will be analyzed in Section 2.4 (Chap. 2) and (approximately) corrected in Section 2.5.

\subsection{A general protocol for fluctuation measurements}

In the general program of extracting nuclear thermodynamics from a fragmentation sample on an event by event basis, we are systematically confronted with the problem of missing information. This concerns detection limitations (neither neutrons nor fragment masses are measured, the response of the experimental filter deforms the events) as well as the uncertainties of the freeze out reconstruction (the side feeding corrections and the freeze out volume are not directly measured) and the unknown physical parameters (the kinetic equation of state Eq. (74) is only an ansatz, and in particular the level density parameter is poorly known at high excitation energy). Since we are interested in first as well as second order moments, the missing information has to be implemented event by event. In principle one would like to restore the missing information in such a way that mean values as well as variances agree with values for the same observables which have been independently measured. This is however in general a very tough task. As an example, one may be able to measure an average value for the size of the fragmenting source through correlation techniques, but these methods always use a whole sample of statistical data and do not give an event by event response.

The general protocol that we have adopted is therefore to fix the average values of the unknown quantities from independent measurements; then the event by event value of the same observables can be fixed such as to systematically minimize the partial energy fluctuations. If the negative heat capacity signal survives, then this can not be ascribed to spurious fluctuation due to unmeasured quantities. It is important to stress that this conservative attitude guarantees the physical meaning of abnormal partial energy fluctuations but prevents a quantitative analysis of the phase transition, in particular a precise evaluation of the latent heat.

The first quantity to be discussed is side feeding. If it is impossible to trace back the evaporative chain on an event by event basis, on the other hand velocity correlations between fragments and light charged particles [66] allow to estimate the average percentage of secondary light charged particles as a function of the excitation energy. This measurement, reported in Figure 41, fixes completely the average multiplicity $M$ at freeze out as well as the average charges of the primary fragments in each excitation energy bin. The very same percentage is associated to each event of the bin, such that the mean value are preserved 

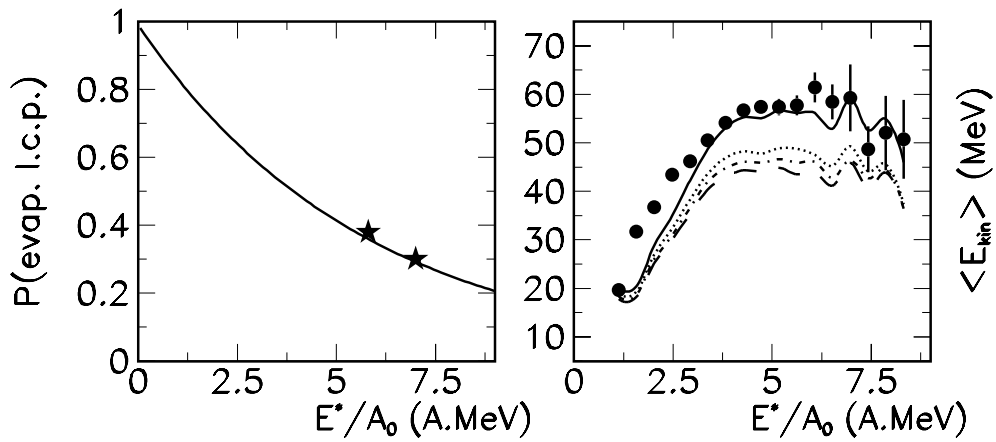

Figure 41. Left part: percentage of evaporated light charged particles as a function of the excitation energy; symbols: experimental data from reference [66]; line: exponential extrapolation. Right part: average fragment kinetic energy for QP data. Symbols: rough experimental data; lines: many body Coulomb trajectory for a volume of $2.5 V_{0}$ (full line), $3 V_{0}$ (dashed), $4 V_{0}$ (dotted), $5 V_{0}$ (dash-dotted), $6 V_{0}$ (long dashed).

while the fluctuations coming from side feeding are minimized. In the same conservative spirit, the extra source of physical (but uncontrolled!) fluctuations given by the number of neutrons at freeze out, has been systematically put equal to zero and no fluctuation has been admitted for the N/Z ratio of the primary fragments.

The same velocity correlations between fragments and particles [66] allow to measure also the average excitation energy of the primary fragments. This experimental information fixes the excitation energy dependence of the level density parameter as shown in Figure 42. Since an important amount of excitation energy is used to break up the excited source (i.e. is found in the measured $Q$ value), the average excitation energy per nucleon of the primary fragments does
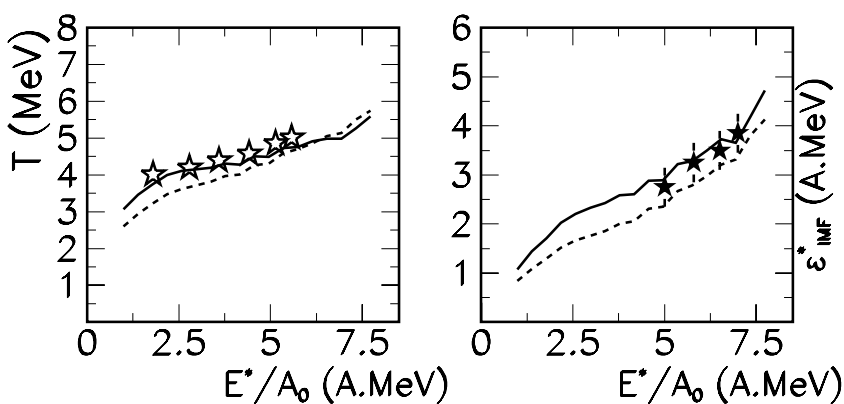

Figure 42. Left: temperature measured with an isotopic thermometer [69] (open symbols) and from equation (74) for the QP (lines). Right: primary fragment internal excitation energy measured from velocity correlations [66] (full symbols) and from equation (74) for the QP (lines). Dashed lines: $a=8$. Full lines: $a=f(A)$ from reference [48]. 
not overcome one half of the total deposited energy. As a consequence of that, no temperature effect is visible in the level density parameter even if the estimated temperature is much lower than a simple Fermi gas estimate, i.e. the limiting source temperature has been attained.

The last parameter entering equations $(65,74,66)$ is the freeze out volume which determines the value of the Coulomb potential energy. Its average value can be determined from the detected average fragment kinetic energy through a many body Coulomb trajectory calculation $[47,67]$. Since the Coulomb energy is positively correlated with the fragment multiplicity, the value obtained for the freeze out volume in each excitation energy bin depends on the side feeding correction. This means that $V_{F O}, a$ and the percentage of evaporated particles have to be fixed consistently with an iterative procedure. When this is done, we find (Fig. 41) that quasi-projectile events are compatible with a value of the freeze out volume close to three times the normal volume and approximately independent of the excitation energy. The deviation at energies lower than $2.5 \mathrm{~A} \mathrm{MeV}$ is not necessarily significative, since more correlation data are needed to constrain the side feeding correction in this excitation energy regime (see left side of Fig. 41).

Of course the experimental results presented in Figures 41 and 42 are subject to uncertainties. However their degree of reliability can be established via cross checks. As an example the symbols in the left panel of Figure 42 show the isotopic temperature [68] from the $C$ thermometer measured for the quasi projectile data [69]. For this specific thermometer, side feeding effects have been estimated to induce an uncertainty of about $0.5 \mathrm{MeV}$ at most [69]. The comparison with the kinetic thermometer gives an error bar on the estimation of the temperature.

This procedure has been applied to peripheral $\mathrm{Au}+\mathrm{Au}$ collisions as well as to central $\mathrm{Au}+\mathrm{C}, \mathrm{Au}+\mathrm{Cu}$ and $\mathrm{Au}+\mathrm{Au}$ events. The result is displayed in Figure 43 [70]. The compatibility of the two sets of data and the clear presence of divergences and a negative branch for $C$ is indisputable. In the case of the central $\mathrm{Au}+\mathrm{Au}$ sample, a collective radial flow component of $1 \mathrm{~A} \mathrm{MeV}$ has been subtracted to the total energy balance based on a comparison with the statistical model SMM [71]. The fragment kinetic energies would also be compatible with zero radial flow and an increased Coulomb repulsion from a more compact freeze out configuration; as it can be seen from Figure 43 this Coulomb versus flow ambiguity already mentioned in Section 2.1 does not change drastically the result.

The same analysis has been performed on central Xe + Sn collisions measured from 32 to $50 \mathrm{~A} \mathrm{MeV}$ with the Indra multidetector $[47,62]$. Similar to the $\mathrm{Au}+\mathrm{Au}$ sample, a radial flow component has been identified in these data sets [62]. In the case of the $32 \mathrm{~A} \mathrm{MeV}$ sample, based on the analysis of the average fragment kinetic energies, the average flow can range from 0.4 to $1.2 \mathrm{~A} \mathrm{MeV}$ depending on the freeze out volume [62]. The results are reported in Figure 44. Independent of the volume assumed, a clear negative heat capacity signal is seen in an excitation energy range which is still statistically significant; however the Coulomb versus flow ambiguity prevents a precise determination of the second divergence of the heat capacity. The comparison between Figures 43 and 44 shows that the different data sets are fully compatible and that the results do not depend on the detector. 

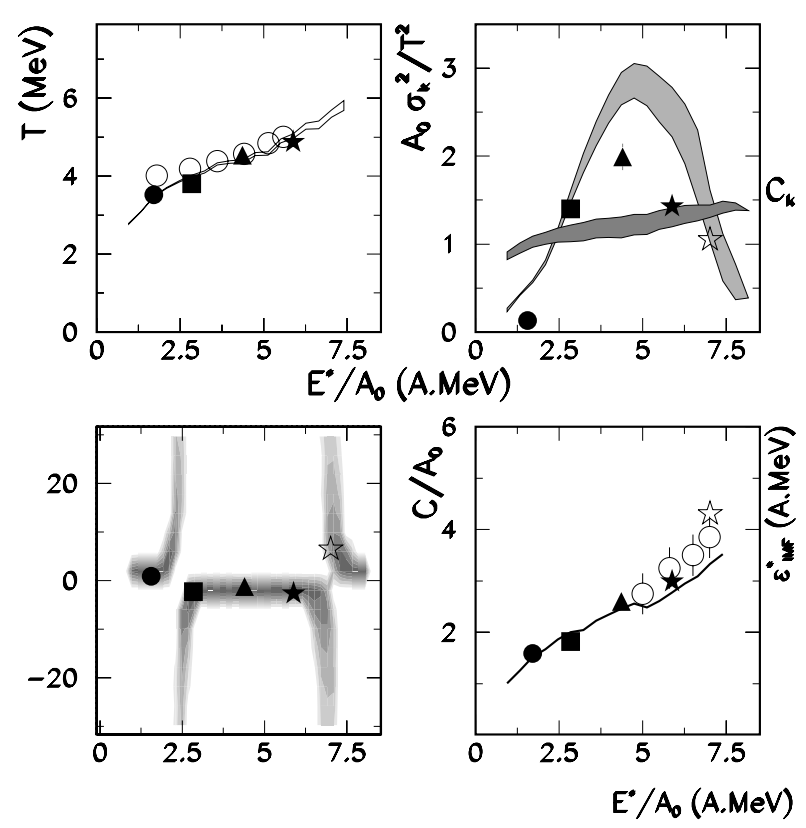

Figure 43. Comparison between the experimental thermodynamic variables after sidefeeding correction for peripheral $\mathrm{Au}+\mathrm{Au}$ (lines and grey contours) and central $\mathrm{Au}+\mathrm{C}$ (black dots), $\mathrm{Au}+\mathrm{Cu}$ (squares and triangles), $\mathrm{Au}+\mathrm{Au}$ with (black stars) and without (open stars) subtraction of $1 \mathrm{AMeV}$ radial flow. Upper left: temperature from equation (74) (line and black symbols) and from the $C$ isotopic thermometer [69] (open symbols). Upper right: normalized partial energy fluctuations and kinetic heat capacity. Lower left: total heat capacity from equation (66). Lower right: internal fragment excitation energy from equation (74) (line and black symbols) and from particles-fragments correlation functions [66]. The grey contours represent the confidence intervals due to the propagation of statistical uncertainties.
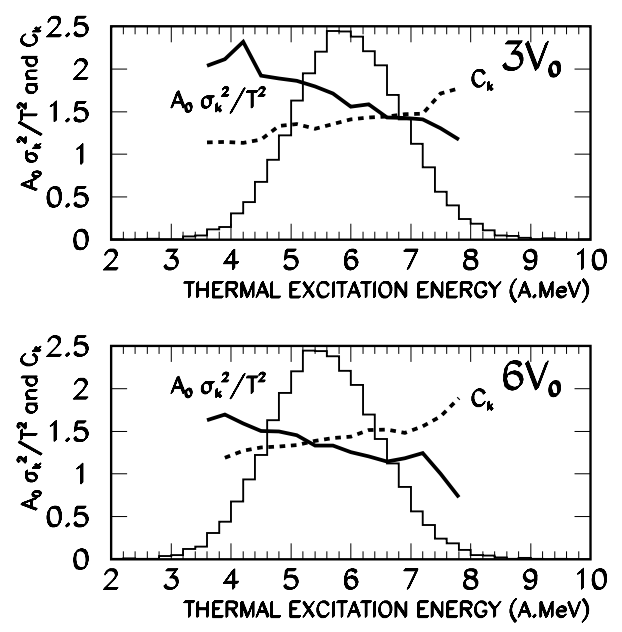

Figure 44. Normalized partial energy fluctuation (full lines) compared to the kinetic heat capacity (dashed lines) and excitation energy distribution (histograms) with the assumption of a minimal (upper part) and maximal (lower part) contribution of the radial flow from central $\mathrm{Xe}+\mathrm{Sn}$ events at $32 \mathrm{~A} \mathrm{MeV}$ from the INDRA collaboration. 


\subsection{Experimental distortions}

In the experimental evaluation of the kinetic energy at freeze out equation (73), the total excitation energy is the result of an event by event calorimetric measurement via

$$
E^{*}=\sum_{i=1}^{N_{c}} m^{(i)}+e_{k}^{(i)}+N_{n}\left(m_{n}+\left\langle e_{k}\right\rangle_{n}\right)-m_{0}
$$

where $N_{c}$ and $N_{n}$ are the charged particles and neutron multiplicities. The calorimetric measurement is affected by a number of uncertainties. The neutron number as well as the neutron energies are not measured; an isotopic resolution is achieved only for light fragments; the experimental filter may deform the energy response, even if this effect is minimized by the analysis of only complete or quasi complete events. Moreover for the analysis of the QP data only the light particles emitted in one half of the total solid angle are kept and their contribution symmetrized to the other half.

To complete this missing information a mass has been assumed for all the detected fragments following the EPAX parametrization; the number of neutrons is then deduced from mass conservation by assuming that the fragmenting source has the same isospin ratio as the composite system (for central events) or as the projectile (for quasi-projectile events). The average energy of neutrons has been obtained from the total detected kinetic energy of the excitation energy bin by means of an effective temperature [72].

The effect of all these approximations can be appreciated using SMM [48] as an event generator. 70000 events are generated from a continuous and flat distribution of excitation energies in the range 1-10 A MeV for an Au source. Figure 45 shows the effect of calorimetry on the two ingredients necessary to calculate the heat capacity. The thick solid lines give the original values of the model. The thin solid line represents the case where both $T$ and $\sigma_{k}$ are calculated from the event by event reconstructed $E_{k}$, but the excitation energy is the input $E^{*}$ of the simulation. The good reproduction of the theoretical temperature is not surprising, since all the unknown parameters have been fixed to reproduce the average quantities. The dramatic decrease of the fluctuations is essentially due to the fact [47] that the multiplicity fluctuations at freeze out are washed out by the secondary decays. This fluctuation suppression is not cured by the side feeding correction because this latter has been implemented in such a way that no extra fluctuations have been introduced (see Sect. 2.3, Chap. 2). Indeed whatever deterministic algorithm we can choose to reconstruct the primary multiplicity, it will not modify the asymptotic measured fluctuations if it is implemented on an event by event basis. This drastic reduction is due to the fact that multiplicity is positively correlated both to the Coulomb potential $V_{c}$ and to the $Q$-value; a reduction in the multiplicity fluctuation will therefore suppress the variances of $V_{c}$ and $Q$ as well as their (positive) covariance. This is why even if we impose a large average value of the multiplicity [47] (within the constraint of an approximate reproduction of $\left.\left\langle E_{k}\right\rangle\right)$, the increase of $\sigma_{k}$ is negligible. 


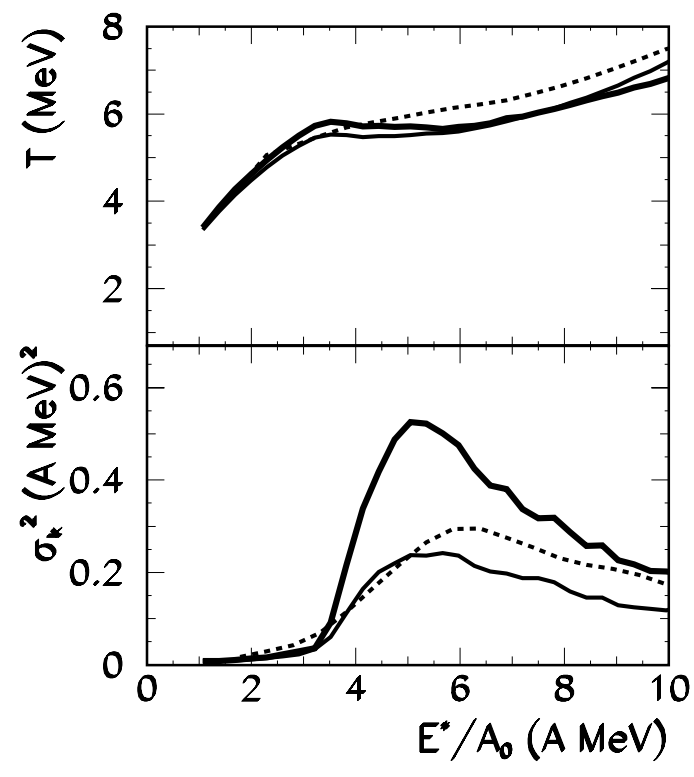

Figure 45. Effect of the calorimetry on the temperature (upper part) and kinetic energy variance (lower part) measurement. Thick solid lines: reference values of the model. Thin solid line: interaction energy reconstructed as in the data, excitation energy from the model. Solid line with black dots: simulation treated as data, including the MULTICS-Miniball filter.

On the other hand it is well-known that the incomplete detection leads to an artificial spread of the excitation energy distribution, a delta function in energy being replaced by a Gaussian with a width of the order of $1 \mathrm{AMeV}$ or even more $[47,73]$. However, data being analyzed in constant excitation energy bins, the variance of the excitation energy reconstruction does not sum up with the configurational energy variance. The spurious width induced by calorimetry is determined solely by the energy bin independent of the width of the calorimetric excitation energy distribution, and this effect has been found to be negligible [47]. However the calorimetric distortion can be at the origin of another spurious effect: a large spread of the measured excitation energy, once it is used as a sorting parameter, can lead to event mixing which can in turn artificially enhance the configurational energy fluctuations. Figure 45 shows that the event mixing due to the imperfect calorimetry does not affect the calculation of average values as the temperature while indeed the variance is somewhat increased and its functional behaviour slightly deformed.

Different important conclusions can be drawn out of Figure 45:

- in the context of the SMM model, our conservative attitude in the reconstruction of the freeze out leads to a reduction in the fluctuations of about a factor two, even in the case in which the calorimetric distortions are taken into account. This means that the experimental observation of abnormal fluctuations can never be due to the experimental limitations, if the average values of $V_{F O}, a$ are fixed from the experimental data themselves;

- this same fluctuation reduction implies that a quantitative reconstruction of the heat capacity is not possible with the present analysis, and in particular 
the latent heat is systematically underestimated. It is important to stress that this conclusion, as well as the previous one, is based on the original values of the SMM fluctuations at freeze out, i.e. it is model dependent;

- the effect of calorimetry can be summarized as an overall $20 \%$ increase of the fluctuations. This model independent result implies that if the experimental fluctuations are only 20\% higher than the kinetic heat capacity, no definitive conclusion can be drawn about the existence of a negative branch for the heat capacity. This is not the case for the experimental data presented in Figure 43.

\subsection{Correcting the systematic errors}

We have shown in the previous section that the way of dealing with the missing experimental information leads to a systematic underestimation of the partial energy fluctuations. In the case of the SMM model the fluctuation suppression can attain a factor two (see Fig. 45). This is essentially due to the fact that secondary decays lead to an overall uniformization of the fragment partitions, and the freeze out fluctuations are not restored by the freeze out reconstruction since side feeding is treated only in average. The constant value assumed for the $N / Z$ ratio of primary fragments and for the number of (undetected) neutrons, the constant multiplicity increase attributed to the secondary decays, are all factors that contribute to decrease the variety of the physical freeze out configurations. The fluctuations are compared to the canonical expectation $C_{k} / T^{2}$ which is solely determined by the average value of the kinetic energy, and therefore does not suffer of the same problem. The net result is that a quantitative estimation of the heat capacity is not possible. This is especially annoying in data samples that, because of the specific entrance channel dynamics and/or the bias of the data selection, contain an important contribution of the so called neck emission, i.e. a directed longitudinal flow reminiscent of the entrance channel [74]. We expect that this out of equilibrium component would decreases the partial energy variance (see Sect. 1.3, Chap. 1) and indeed no abnormal fluctuations are observed in these sets of data [75].

The deformation of the fluctuation signal can be estimated in a model independent way by successively applying the freeze out reconstruction and experimental filter to the data [70]. A freeze out configuration is reconstructed from the measured partitions as in Section 2.3 (Chap. 2) above; this configuration is then made decay with the evaporation code SIMON based on standard Weisskopf decay rates leading to a new asymptotic partition which is again treated as experimental data, and the procedure can be iterated further. The charge distribution after SIMON is fully compatible with the measured distributions; moreover the mean value of the kinetic energy at freeze out does not change from an iteration to the other [70]. This shows that the freeze out reconstruction and the experimental filter do not deform the average response. On the other hand the partial energy fluctuations are modified and saturate when the freeze out reconstruction 


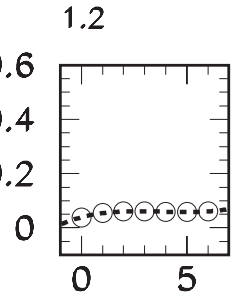

4.8

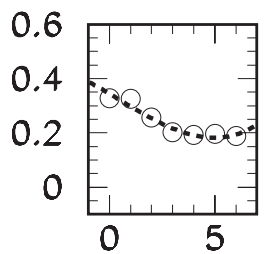

2.4

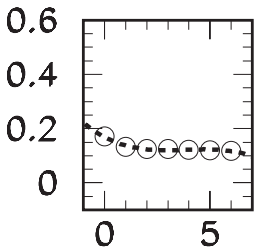

6

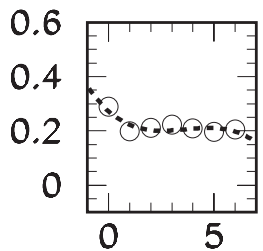

3.6

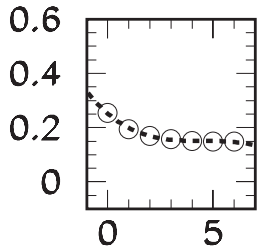

7.2

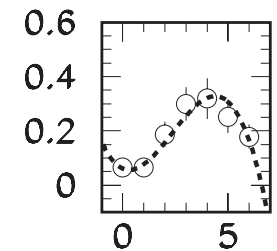

Figure 46. Partial energy fluctuations as a function of the freeze out reconstruction iterations in different excitation energy bins for the Au QP data. The zero of the abscissa corresponds to the first iteration, -1 is the extrapolation to the freeze out. Dashed line: polynomial fit.

procedure converges, as it can be seen in Figure 46. In the first and last excitation energy bin the freeze out reconstruction overestimates the fluctuations of the previous iteration: in this small fluctuation regime secondary decays lead to the exploration of new configurations, i.e. increase the partial energy variance. In the last bin, no monotonous behaviour is observed: the statistics is too low here to make any positive conclusion on the value of the heat capacity. In the excitation energy interval $2<E^{*}<6 \mathrm{~A} \mathrm{MeV}$ the freeze out reconstruction systematically underestimates the fluctuations, similar to the case of the SMM model in the last section. The actual value of the fluctuations at freeze out can be obtained by extrapolating back to iteration zero the fluctuation distortion (dashed line in Fig. 46).

The final result for the partial energy fluctuations in the Au QP sample is given by the full black line of Figure 47. A linear fit of the two first iterations displayed in Figure 46 is also shown. The evaluation of the confidence interval on this result is in progress [70]. However it is clear that, independent of the extrapolation technique, the fluctuation peak overcomes the reference value that we have estimated in Section 2.3 (Chap. 2) as $\sigma_{c a n}^{2}\left(E^{*}=4.5\right)=0.13 \pm 0.03$ (see Eq. (57)). This confirms the observation of negative heat capacity in nuclear multifragmentation [47] and we can consider this result as the first quantitative measurement of the thermodynamics of a hot gold nucleus.

It is interesting to remark that the procedure sketched in this section, applied to filtered SMM simulations allows to reconstruct the original freeze out fluctuations within a few percent [70]. 


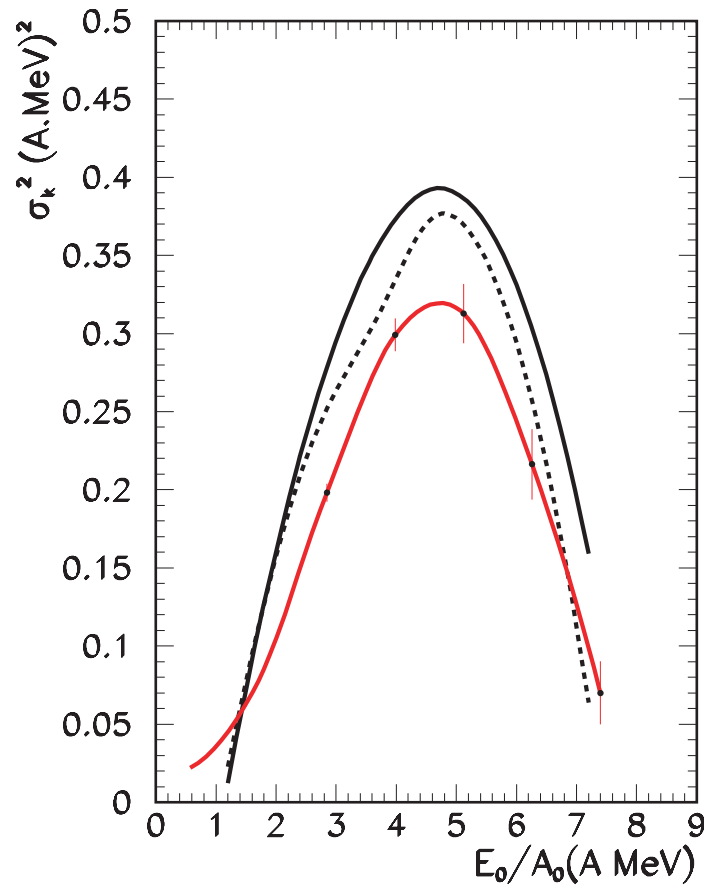

Figure 47. Partial energy fluctuations before (red line) and after (black lines) the fluctuation correction for the $\mathrm{Au}$ QP data. Full black line: polynomial interpolation of the iterative fluctuation distortion Figure 46. Dashed black line: linear fit between the two first iterations.

\section{Coulomb effects}

The analogy between a Van der Waals gas and nuclei is broken by the presence in these latter of the long range Coulomb interaction; the non-saturating character of the force prevents the definition of a thermodynamic limit and gives rise to a size dependent limiting temperature [76] above which nuclei cannot exist as bound systems. The effect of the Coulomb interaction on the phase transition has been the subject of numerous investigations [77] and, based on an analysis of the latent heat, it has been argued that above a critical size the phase transition may disappear. In this chapter we shall propose a systematic way to perform the link between the statistical properties of charged and uncharged systems. The result will be that the phase transition can be traced from a concavity anomaly of the very same well defined thermostatistical potential, independent of the fact that the Coulomb interaction is active or not. The effect of Coulomb can be understood as a deformation of the probability distribution of events in the space of observables, corresponding to a rotation of the order parameter. When the system is small and Coulomb represents a minor correction in the global energetics, the equations of state of the uncharged system can be (at least in principle) exactly reconstructed from the distribution of charged events, while if the system is heavily charged, the distortion is such that new partitions inaccessible to the uncharged system become dominant and the disappearance of the latent heat [77] can be interpreted as a cross-over. 


\subsection{The multi-canonical ensemble}

To explore the effect of the Coulomb interaction we can introduce an additional observable

$$
V_{c}=\sum_{i \neq j} \frac{Z_{i} Z_{j}}{\left|\vec{r}_{i}-\vec{r}_{j}\right|},
$$

which, similarly to $\left\langle R^{3}\right\rangle$ (see Sect. 1.2, Chap. 2), is a measure of the compactness of the system, and in the case of charged systems is proportional to the Coulomb energy $E_{c}=\alpha_{c} V_{c}$. The total energy of the system $E$ can thus be splitted into two terms, $E_{\text {tot }}=E_{n}+E_{c}=\alpha_{n} V_{n}+\alpha_{c} V_{c}$, where $\alpha_{n}$ is the nuclear coupling, or more generally the coupling associated to the short range component of the interaction (for the Ising model, $\alpha_{n}=J$ ), and $V_{n}=E_{n} / \alpha_{n}$ is the associated observable.

If the system is in contact with a heat bath (canonical ensemble), we can sort the events as a function of both $V_{n}$ and $V_{c}$ :

$$
p_{\beta}\left(V_{n}, V_{c}\right)=\frac{1}{Z_{\beta}} W\left(V_{n}, V_{c}\right) e^{-\beta \alpha_{n} V_{n}-\beta \alpha_{c} V_{c}} .
$$

The canonical total energy distribution is an integration of the generalized distribution (75): $p_{\beta}\left(E_{t o t}\right)=\int d V_{c} p_{\beta}\left(E_{t o t} / \alpha_{n}-\alpha_{c} / \alpha_{n} V_{c}, V_{c}\right)$. Equation (75) naturally leads to the definition of a generalized entropy $S\left(V_{n}, V_{c}\right)=\log W\left(V_{n}, V_{c}\right)$. The standard entropy can be obtained by a projection of the density of states on the total energy axis

$$
W_{t o t}\left(E_{t o t}\right)=\int d V_{c} W\left(\frac{E_{t o t}}{\alpha_{n}}-\frac{\alpha_{c}}{\alpha_{n}} V_{c}, V_{c}\right) \text {. }
$$

One can also notice that the projection of $W\left(V_{n}, V_{c}\right)$ on the $V_{n}$ axis provides the entropy of the uncharged system

$$
S_{n}\left(V_{n}\right)=\log W_{n}\left(V_{n}\right)=\log \int d V_{c} W\left(V_{n}, V_{c}\right) .
$$

Since we have introduced two observables $\left(V_{n}, V_{c}\right)$, it is natural to extend the canonical ensemble by introducing two Lagrange multipliers $\beta_{n}, \beta_{c}$ leading to the distribution of events in the two-dimensional observables space

$$
p_{\beta_{n} \beta_{c}}\left(V_{n}, V_{c}\right)=\frac{1}{Z_{\beta_{n} \beta_{c}}} W\left(V_{n}, V_{c}\right) e^{-\beta_{n} V_{n}-\beta_{c} V_{c}},
$$

where $Z_{\beta_{n} \beta_{c}}$ is the partition sum of this multi-canonical ensemble corresponding to the independent observation of the two components $V_{n}, V_{c}$ of the energy.

In the cases $\beta_{c}=\beta_{n} \alpha_{c} / \alpha_{n}$ and $\beta_{c}=0$, equation (76) reduces to the usual canonical ensemble when the Coulomb interaction is, or is not taken into account. All intermediate values $0<\beta_{c}<\beta_{n} \alpha_{c} / \alpha_{n}$ correspond to physical systems in which the charge is partially screened $\left(0<\alpha_{c}^{\text {eff }}=\alpha_{n} \beta_{c} / \beta_{n}<\alpha_{c}\right)$, and give rise to interpolating ensembles between the charged and the uncharged case, in the same 
way as the Gaussian ensemble [12] gives a continuous interpolation between the microcanonical and the canonical ensemble.

The multicanonical ensemble can thus be considered as the canonical ensemble at a temperature $\bar{\beta}^{-1}=\alpha_{n} / \beta_{n}$ of particles charged with an effective charge $\bar{q}^{2} / q^{2}=$ $\beta_{c} / \beta_{n}$. In fact we can introduce an effective energy as $E_{\text {eff }}=E_{n}+\alpha_{c}^{\text {eff }} V_{c}$. Then the distribution of the effective energy is a canonical distribution with a Boltzmann factor $e^{-\bar{\beta} E_{e f} \text {. }}$.

Considering $\beta_{c}=0$ (or $\beta_{c} \rightarrow 0$ faster then the increase of the charge when the volume of the system is increased), the multicanonical ensemble is also a way to study the thermodynamic limit.

In the case of nuclear physics at equilibrium, the only physical ensemble is of course the $\alpha_{c}^{\text {eff }}=\alpha_{c}$ one, however it is important to remark that all the other ensembles can be experimentally accessed if the different parts of the energy are not equilibrated. This conjecture may not be irrealistic for the specific energy sharing $E_{\text {tot }}=E_{n}+E_{c}$ since the non-Coulomb part of the energy comes from a short range interaction; indeed it is well-known that the relaxation time depends critically on the range of the force [78].

Let us now focus on the question of (first order) phase transitions by studying the convex region of the entropy $S\left(V_{n}, V_{c}\right)$. Equation (76) clearly demonstrates that the convexity anomalies of the entropy $\log W$ can be traced back from $\log p_{\beta_{n} \beta_{c}}$. Indeed, the curvature matrix of the entropy and of the probability distribution are the same

$$
C_{k k^{\prime}}\left(V_{c}, V_{n}\right) \equiv \frac{\partial^{2} \log W\left(V_{c}, V_{n}\right)}{\partial V_{k} \partial V_{k^{\prime}}}=\frac{\partial^{2} \log p_{\beta_{n} \beta_{c}}\left(V_{c}, V_{n}\right)}{\partial V_{k} \partial V_{k^{\prime}}}
$$

These relations are valid for every set of Lagrange multipliers $\beta_{n}, \beta_{c}$ because in a finite system the probability distribution covers the entire accessible observables space. This means that the charged and uncharged system can be analyzed in a unique framework and the equations of state of the one can be exactly calculated from the event distribution measured in the other. For example, the uncharged system entropy can be deduced within a constant from the statistical distribution of the charged system by projecting out the Coulomb energy after a proper Boltzmann weighting

$$
S_{n}\left(V_{n}\right)=\log \left(\int d V_{c} e^{\beta_{c} V_{c}} p_{\beta_{n} \beta_{c}}\left(V_{n}, V_{c}\right)\right)-\beta_{n} V_{n}
$$

The only limitation can be a practical one for experiments or simulations: to accumulate enough statistics at every location. The phase transition is defined by the convexity properties of the thermodynamic potential $\log W$ (see Sect. 4, Chap. 1) which is the same for the charged and uncharged case: the existence and order of the phase transition cannot therefore be modified by the consideration of the Coulomb interaction. However the population $p_{\beta_{n} \beta_{c}}$ explicitly depends on $\beta_{c}$, i.e. on the presence (or absence) of Coulomb. It is therefore possible that the region of anomalous curvature is explored in a different way by the charged system, if the Coulomb energy is in equilibrium with the nuclear part. 


\subsection{The statistical multifragmentation model}

In order to illustrate these general ideas and make some connections to the nuclear multifragmentation experiments, we have made some calculations in the multi-canonical ensemble with a statistical multifragmentation model [79]. The model describes a multifragmentation event as an ensemble of spherical, non overlapping fragments with ground state energies parametrized from a liquid drop model, a Fermi gas distribution for the internal energies with a high energy cut-off, interacting through their mutual Coulomb repulsion. The translational degrees of freedom are treated classically, and the model is completed with a secondary evaporation step [79] to evacuate the residual excitation energy of the fragments. Each event is characterized by its total mass and charge number (i.e. the size of the disassembling nuclear source), its total energy that can be decomposed in a Coulomb $E_{c}$ part that includes also Coulomb effects on the binding energy, and a non Coulomb one that corresponds to the nuclear interaction inside fragments plus the translational energy. In actual simulations, when we evaluate the Coulomb energy we introduce an effective charge $\bar{q}$ that is tuned from $\bar{q}=0$ to $\bar{q}=1$ to compute the different possible states of charge of the system. Each event can also be associated to a spatial extension, given by the freeze-out volume that contains all the fragments. This volume being a fluctuating quantity in any system without boundary conditions (see Sect. 1.2, Chap. 2), it is treated as an extra observable associated to an additional Lagrange multiplier $\lambda$. The integration of the total number of states of the system over fragment momenta can be analytically performed leading to a specific statistical weight for each partition in a smaller configuration space from which the momentum observables have been projected out [79]. Using these weights, a Metropolis-type simulation is employed in order to generate a microcanonical ensemble of events that finally gives the multi-canonical statistical ensemble according to equation (76). The resulting distribution of events can then be plotted in the $\left(V_{n}, V_{c}\right)$ plane or equivalently in the $\left(E_{t o t}, E_{c}\right)$ plane. When the Coulomb interaction is at play $(\bar{q}=1)$ the total energy is simply the $E_{\text {tot }}$ axis. When the Coulomb energy is not effective $(\bar{q}=0)$ the relevant energy axis is the $E_{t o t}-E_{c}$ one i.e. the second bisector. In an actual heavy ion experiment the dynamics of the collision and the selection criteria usually lead to a sample with a relatively well defined excitation energy; such an approximately microcanonical sample corresponds to a simple cut of the bidimensional distributions.

\subsection{Coulomb interaction and the topology of events}

Figure 48 shows the distribution of events for a system of 50 particles at a subcritical pressure $\lambda_{V}=3 \times 10^{-4} \mathrm{fm}^{-3}$ in the Coulomb energy $E_{c}$ and total energy $E_{\text {tot }}$ (calculated respect to the ground state of the disassembling source) plane. The "charged" case $\beta_{c}=\alpha_{c} \beta_{n} / \alpha_{n}$ (i.e. when the Coulomb energy is observed at equilibrium with the nuclear energy) and the "uncharged" one $\beta_{c}=0$ are displayed. Let us look at the uncharged case first. The topology of events is characteristic of 


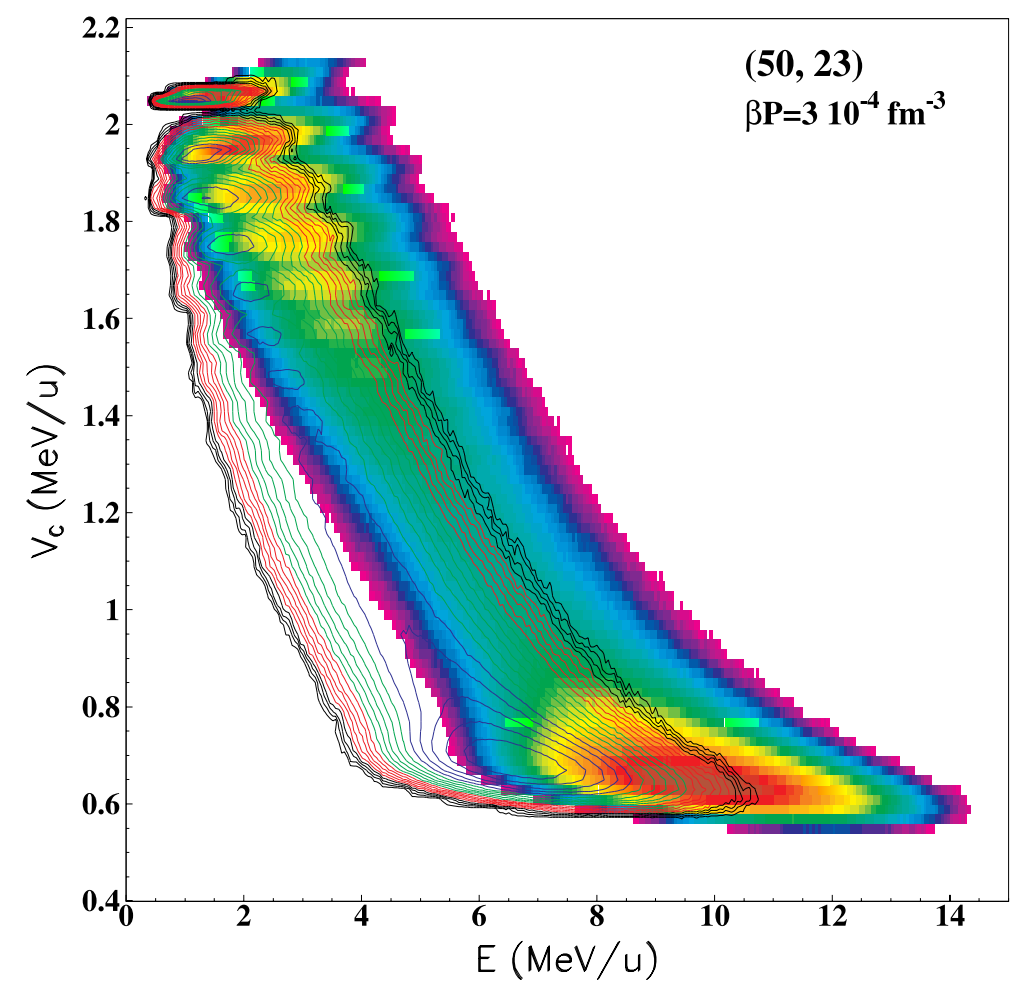

Figure 48. Subcritical event distribution in the Coulomb energy versus total energy plane for a system of total mass number $A=50$ and atomic number $Z=23$ in the isobaric multicanonical multifragmentation model. Contour plot: $\alpha_{n} / \beta_{n}=3.7 \mathrm{MeV}, \beta_{c}=0$; contour lines: $\alpha_{c} / \beta_{c}=\alpha_{n} / \beta_{n}=3.1 \mathrm{MeV}$.

a first order phase transition, with an accumulation of events at low excitation energy and high Coulomb energy (compact configurations or "liquid" phase) and an accumulation at high energy and low Coulomb (rarefied configurations or "gas" phase). The projection over the energy axis is bimodal; the distance between the two peaks measures the latent heat of the transition, while the region of inverted curvature is related to a negative heat capacity in the microcanonical ensemble. The representation in the $\left(E_{c}, E_{t o t}\right)$ plane reveals however a much more detailed structure than the more standard projection over the energy axis. Indeed the liquid peak is constituted by a series of structures or channel openings, associated to similar excitation energies but corresponding to different charge partitions. In the condensed matter community it is customary to define isomeric transitions as phase transitions [17]. In this spirit the peaks observed in Figure 48, that are clearly resolved in the projection over the $E_{c}$ axis, can in principle all be defined as "phase transitions", in the loose sense that they lead to a convex intruder of the associated entropy. However it is important to remark that the configurations 
that belong to the different peaks of the $E_{c}$ distribution differ in average for one or a few particles, i.e. do not correspond to a collective change of state at variance with the "liquid" and "gas" peak appearing in the energy distribution that correspond to a complete rearrangement of the charge partition of the system and would survive in the bulk limit (see Sect. 4.5, Chap. 1). A similar situation has been experimentally found in reference [80], where a thermodynamic study of nuclei in the rare earth region has revealed at very low excitation energies a series of backbendings in the entropy, interpreted as channel openings and attributed to the breaking of single pairs of nucleons. Only the ensemble of these structures leads to a global change of slope in the equation of state, which is then interpreted as a signature of the transition from superfluid to normal fluid [80]. In the same way we prefer to consider the structures of Figure 48 as the ensemble of the single particle state modifications that constitute the microscopic origin of the global collective phase change that can be considered as a veritable phase transition.

The event distribution of the charged system in Figure 48 shows globally the same structure as in the uncharged case, with two important differences. First, to have the same height in the two relative maxima, the temperature has been lowered. The decrease of the transition temperature is due to the repulsive character of the Coulomb interaction $[76,81]$. Second, even if the phase transition is still clearly visible in the bidimensional representation, this is not true any more for the projection over the energy axis. The usual interpretation of Figure 49 would be $[76,81]$ the expected lowering of the critical point due to the Coulomb interaction. The complete bidimensional information of Figure 48 demonstrates that this conclusion is not completely correct. Indeed, while the event distribution is almost unchanged, the projection axis is different in the charged case and the best order parameter (i.e. the direction that separates at best the two phases) is now almost perpendicular to the energy direction. Introducing a better observable playing the role of the order parameter, $O=a E+b V_{c}$ with $a \ll b$, would thus restore the overall picture of the first order phase transition even in the charged system.

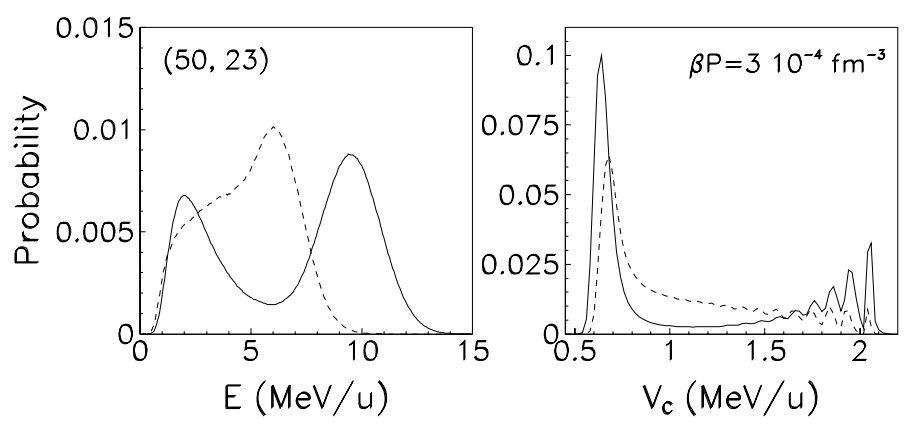

Figure 49. Projections of Figure 48 above over the Coulomb energy and total energy axes. Dashed (full) lines: the Coulomb interaction is (is not) observed at equilibrium with the nuclear energy. 
The effect of an electric charge is a modification of the probability distribution in the observables space that does not affect the convexity properties of the associated entropy, these latter being independent of $\lambda_{V}, \beta_{n}, \beta_{c}$, i.e. of the pressure, temperature and state of charge of the system. From the viewpoint of the (multi)canonical ensemble, the presence of the Coulomb interaction implies a modification of the transition temperature and pressure and a rotation of the optimal order parameter; from the viewpoint of the microcanonical ensemble in the $\left(E_{n}, E_{c}\right)$ plane it is just a re-weighting in the occupation probabilities of the very same entropy surface. This in particular implies that the whole thermodynamics of the uncharged system can be (at least in principle) completely reconstructed from the only knowledge of the distribution probability of the charged system at an arbitrary temperature and pressure, by simple re-weighting of the different energy states. Indeed if we consider a charged system at the temperature $\beta_{1}^{-1}$ described by the distribution $p_{\beta_{1}}^{c}$, the distribution $p_{\beta_{2}}^{n}$ at the temperature $\beta_{2}^{-1}$ in the case where the Coulomb interaction is disregarded is given within a normalization by

$$
p_{\beta_{2}}^{n}\left(V_{n}, V_{c}\right) \propto p_{\beta_{1}}^{c}\left(V_{n}, V_{c}\right) e^{-\left(\left(\beta_{2}-\beta_{1}\right) V_{n}-\beta_{1} V_{c}\right)} .
$$

Of course this reconstruction is possible only for the energy states where the population sample is statistically significant; if the probability distributions are concentrated in very different locations in the observables space the size of the sample needed will become increasingly (and soon prohibitively) high.

This more complicated occurs in the case of heavily charged systems, for which the Coulomb distortions get more appreciable. Figure 50 shows the event distribution as in Figure 48 above, but for a much heavier system composed of $A=200$ particles and $Z=82$ charge units. When the Coulomb interaction is neglected the situation gets closer to the expected behaviour in the bulk: the channel openings are not recognizeable any more, and only the collective liquidlike to gas-like state change survives, with the minimum between the two phases getting deeper. If Coulomb is switched on, the event topology drastically changes. A much smaller portion of phase space is populated by any calculation at a single temperature, and this stays true if the pressure is changed. The event distribution describes a curved trajectory that for each type of configuration (i.e. each $E_{c}$ ) minimizes the total energy thus avoiding the concavity anomaly region, i.e. turns around the first order phase transition without diving into the coexistence region. The external edge of the anomalous curvature region is only touched by the calculations at the lowest temperature, pointing to an order parameter almost perfectly perpendicular to the energy direction. These two residual bimodalities correspond to the opening of asymmetric fission (distribution centered on $V_{c} \approx 3$ ) and multifragmentation (distribution centered on $V_{c} \approx 2.5$. Figure 50 indicates that the phase transition in heavily charged systems has to be looked for in directions other than the total energy deposited in the system and rather connected to the different charge partitions. Indeed we will discuss in the next chapter that a bimodality has been observed in multifragmentation experiments [38] in the 


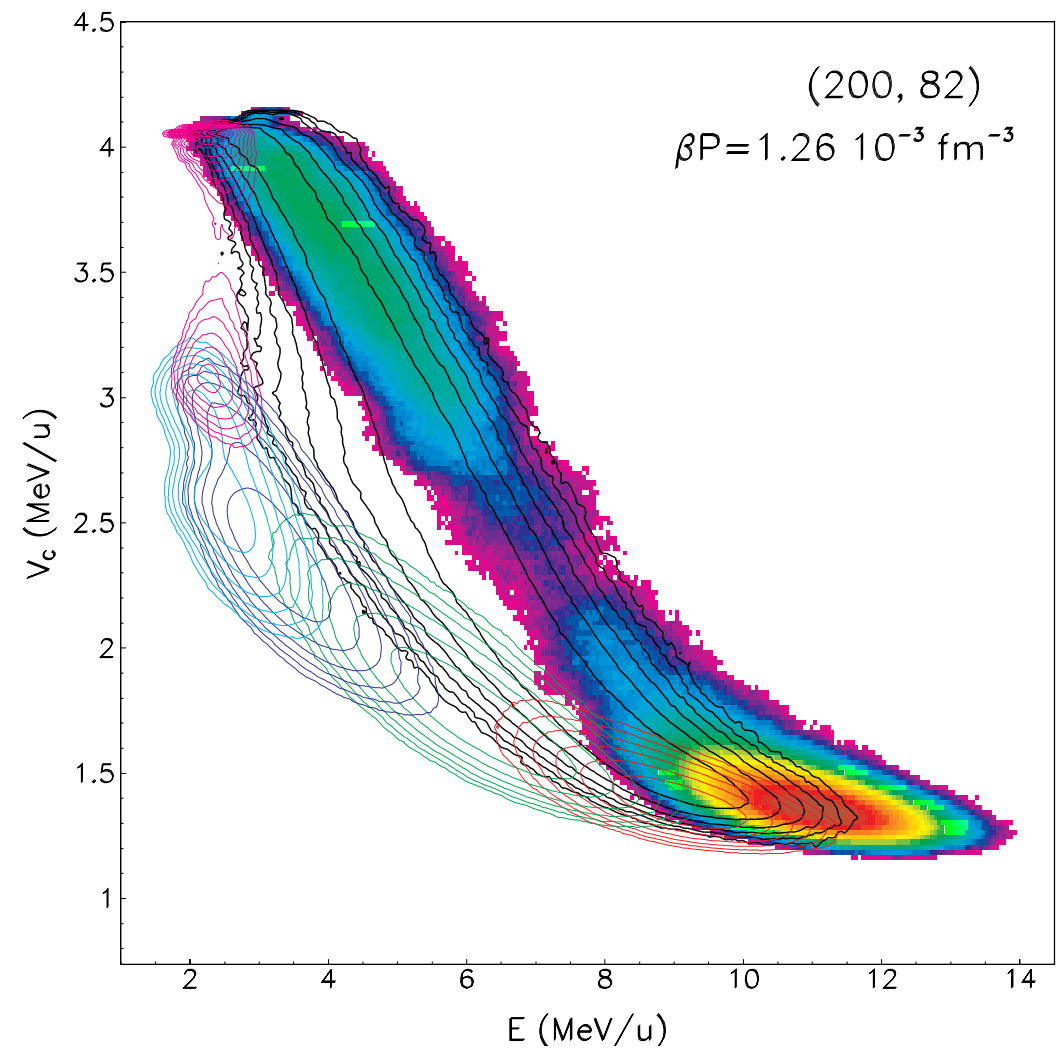

Figure 50. Event distribution in the Coulomb energy versus total energy plane with $\beta P=1.26 \times 10^{-3} \mathrm{fm}^{-3}$ for a system of total mass number $A=200$ and atomic number $Z=82$ in the isobaric multi-canonical multifragmentation model. Contour plot: $\alpha_{n} / \beta_{n}=5.6 \mathrm{MeV}$, $\beta_{c}=0$; coloured contour lines: $\alpha_{n} / \beta_{n}=\alpha_{c} / \beta_{c}$ at temperatures varying from 3 to $4.8 \mathrm{MeV}$; black contours: partially screened Coulomb.

observables plane $\left(Z_{1}, Z_{2}\right)$, where $Z_{1}\left(Z_{2}\right)$ is the biggest (second biggest) fragment measured in every event.

\section{Other signals of phase transitions}

\subsection{Distribution of the order parameter}

We have already discussed in Section 6.6 (Chap. 1) that the biggest fragment detected in each event, because of its correlation with the particle density which is the natural order parameter of the liquid gas phase transition, is a good candidate as a potential order parameter (see also [82]). If the volume of the system is not constrained with sharp boundary conditions, the bimodality in $A_{\max }$ observed 


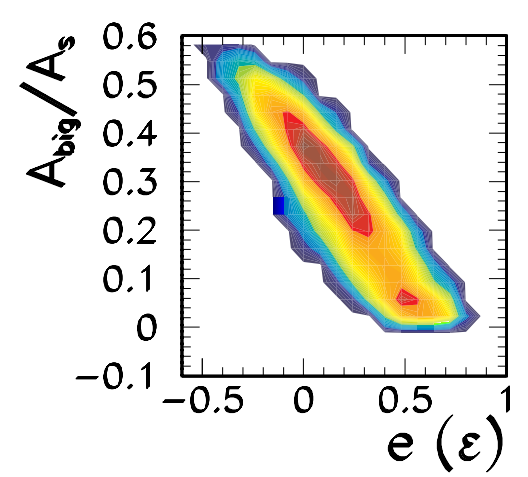

Figure 51. Size of the biggest fragment normalized to the size of the system versus total energy in the canonical isobar lattice gas model at the transition temperature for a system of 216 particles.

in Figure 25 is not suppressed by the requirement of total mass conservation, as shown in Figure 51. This means that, leaving aside for the moment the possible Coulomb distortions (see last chapter), it should be possible to observe a bimodal $A_{\max }$ distribution in multifragmentation experiments if we deal with a sample close to the transition temperature.

Quasi projectile events have been selected from $\mathrm{Au}+\mathrm{Au}$ collisions, studied at GSI by the INDRA and the Aladin collaborations, with a beam energy from 60 to $100 \mathrm{~A} \mathrm{MeV}$. The bimodality is suppressed if the order parameter is constrained by a conservation law, meaning that one has to constrain the total energy of the sample as little as possible. For this reason data are sorted in bins of transverse energy of light charged particles emitted by the quasi-target. The quasi target had been much bigger than the quasi projectile, this sorting would be equivalent to a canonical one. In our case we are rather dealing with a Gaussian ensemble [12], but this sorting has still the advantage to allow large energy fluctuations and ensure a decorrelation between the quasi projectile observables and the sorting criterium, thus minimizing the experimental drawbacks.

An example of the event distribution is represented in Figure 52 in the plane $Z_{\text {max }}$ versus $\operatorname{var}_{\text {symm }}$, where $\operatorname{var}_{\text {sym }}=\left(Z_{\text {max }}-Z_{\text {max }-1}\right) /\left(Z_{\text {max }}+Z_{\text {max }-1}\right)$ measures the charge asymmetry between the two heaviest fragments. In the case of fission events (which can be easily identified because of their specific kinematics) $Z_{\max }$ is defined as in Section 2 (Chap. 2) as the sum of the two fission fragments charges. Fission is reconstructed in order to consider on the same footings the two mechanisms referring to normal density nuclear matter (residue production and fission), and to avoid the physical but relatively trivial bimodality connected to the opening of the fission channel that we have observed in Section 3.3 (Chap. 2). A clear bimodal behaviour is observed at all bombarding energies. The average energy deposited in the bimodal sample (measured in terms of total kinetic energy loss) is an increasing function of the bombarding energy [83] which indicates a non vanishing memory of the entrance channel. If however only compacts events 


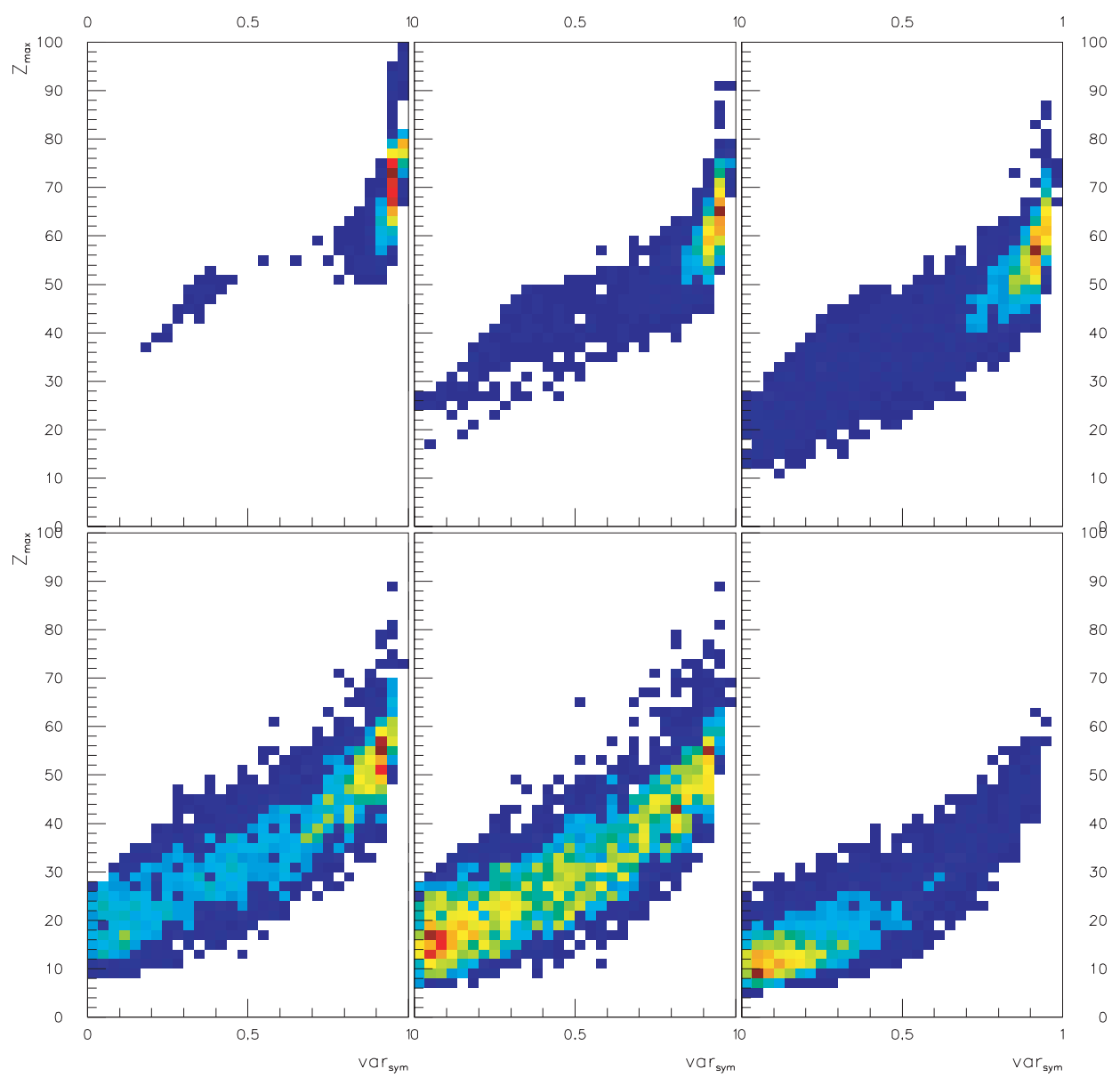

Figure 52. Charge of the biggest fragment as a function of the charge asymmetry $\operatorname{var}_{\text {sym }}=$ $\left(Z_{\max }-Z_{\max -1}\right) /\left(Z_{\max }+Z_{\max -1}\right)$ for Au quasi-projectiles produced in $\mathrm{Au}+\mathrm{Au}$ collisions at $100 \mathrm{AMeV}$, in bins of light charged particles transverse energy emitted from the quasi target [83].

are retained, in which the relative momentum of the heaviest fragment and the center of mass of the others is small, the $Z_{\max } / v r_{\text {sym }}$ correlation is similar to Figure 52 but the average deposited energy becomes independent of the beam, in agreement with the results of Section 1.3 (Chap. 2). The bimodality signals appears very robust respect to out of equilibrium effects.

Figure 53 shows the fluctuation analysis (see Sect. 2, Chap. 2) at $80 \mathrm{~A} \mathrm{MeV}$. The normalized fluctuations do not overcome the canonical expectation, and the peak value decreases with increasing bombarding energy [83], which may be a sign of a collective flow reminiscent of the entrance channel (see Sect. 1.3, 


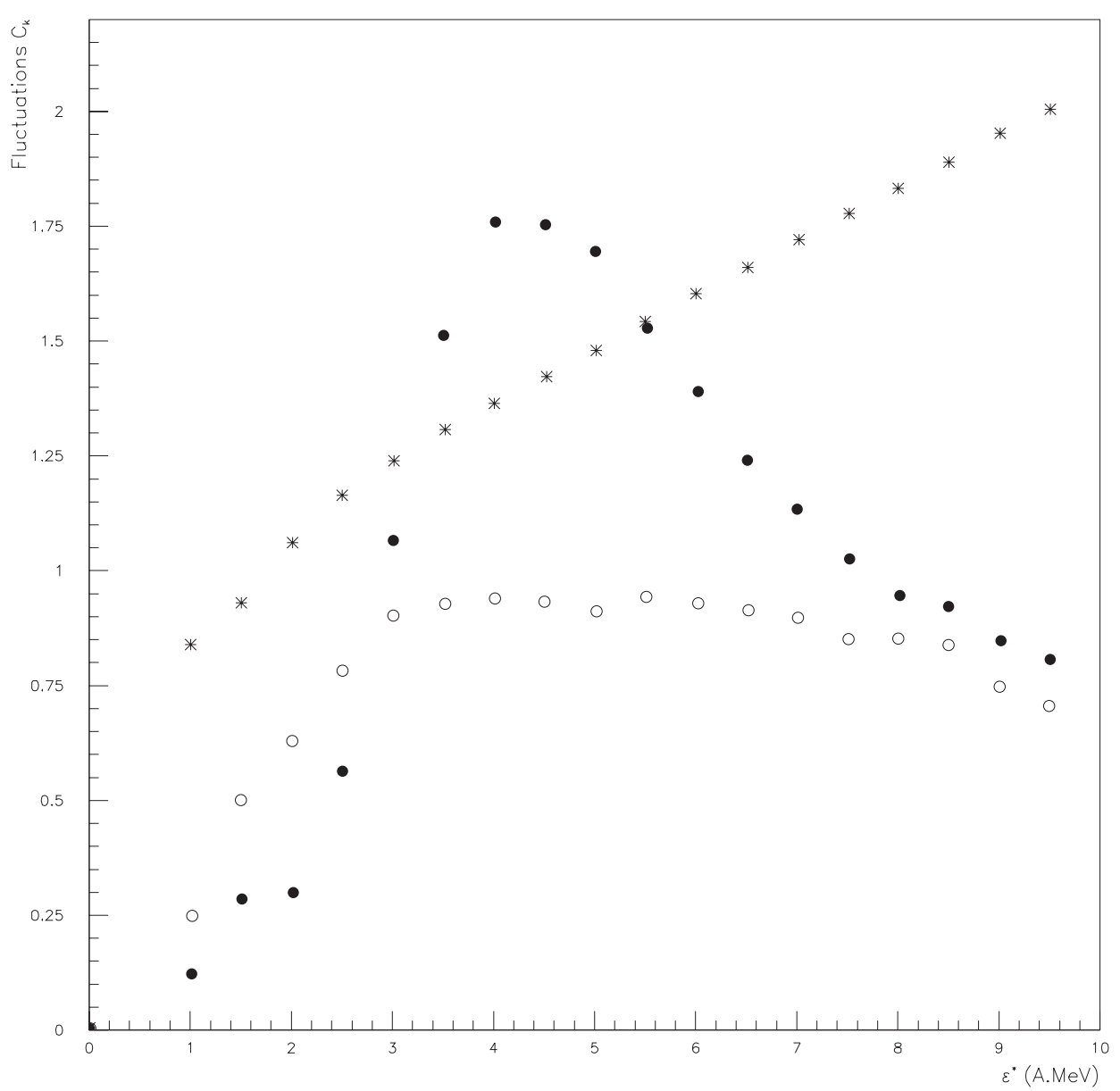

Figure 53. Kinetic heat capacity (stars) and normalized partial energy fluctuations versus excitation energy for all quasi projectile events (open symbols) and after subtraction of events relaxed along the beam axis (full symbols) for Au quasi-projectiles produced in $\mathrm{Au}+\mathrm{Au}$ collisions at $80 \mathrm{~A} \mathrm{MeV} \mathrm{[83].}$

Chap. 2). When only compact events are retained, the fluctuations increase over the canonical heat capacity. For these events the fluctuation value is independent of the entrance channel within error bars [83], which again is an indication of equilibrium. Figure 54 shows the same analysis when the calorimetric sorting is done separately in the different centrality bins. The agreement between the different transverse energy samples when the sorting variable is the total excitation energy is again an indication of microcanonical equilibrium. It is also interesting to note that the whole abnormal fluctuation interval is mapped by the transverse energy bin corresponding to the bimodal distributions (stars) in agreement with the theoretical expectations, showing that the transverse energy sorting indeed 


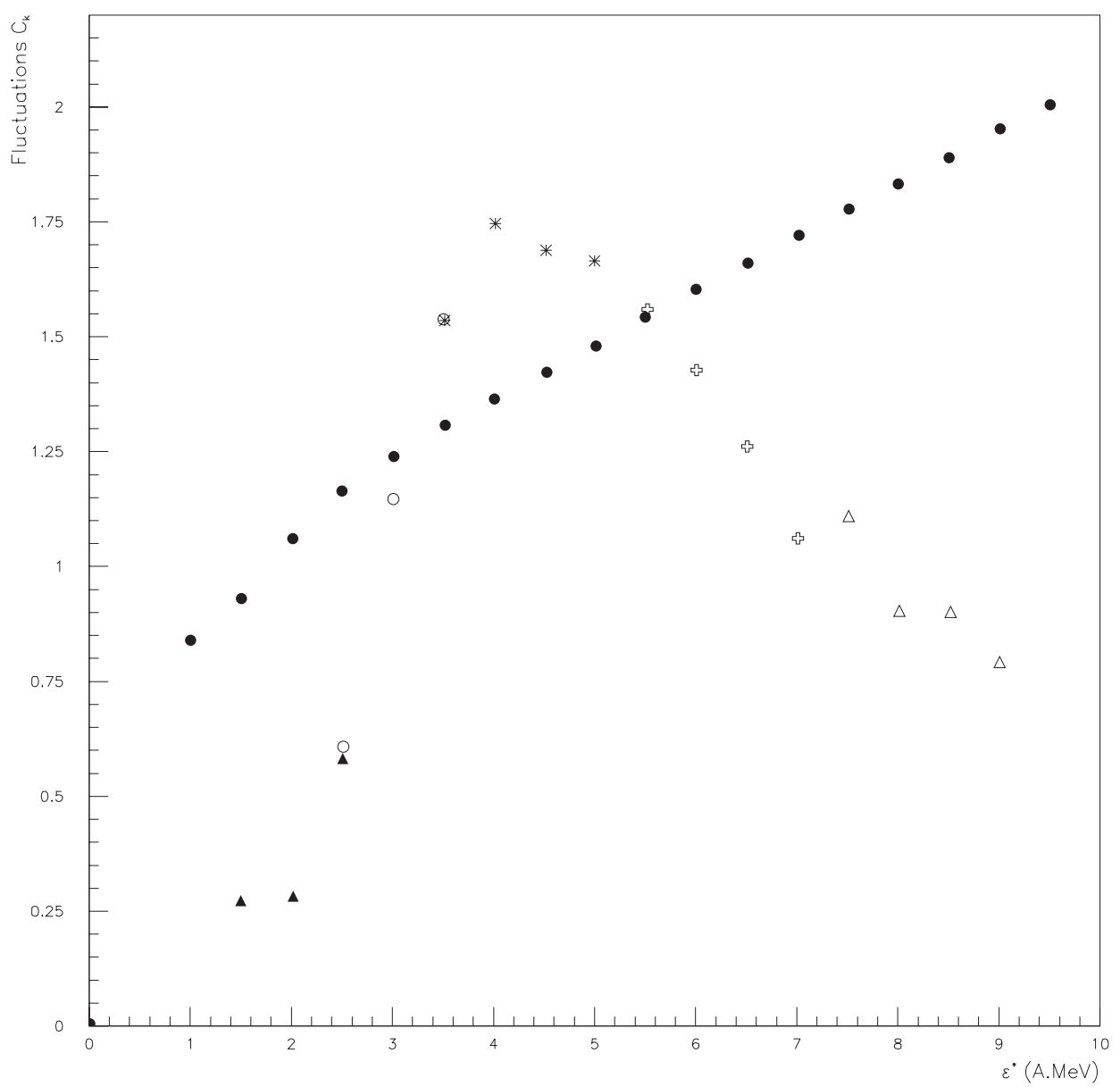

Figure 54. As Figure 53, but for events selected in light charged particles transverse energy bins on the quasi-target side. black triangles: $0<E_{t}<50$; circles: $50<E_{t}<150$; stars: $150<E_{t}<250$; crosses: $250<E_{t}<350$; open triangles: $350<E_{t}<450$. In all cases only compact events minimizing the memory of the entrance channel are retained. The bimodality in the $Z_{m a x}, \operatorname{var}_{\text {sym }}$ plane is observed in the third bin [83].

allows huge energy fluctuations. All these features are compatible with a scenario in which multifragmentation represents the coexistence phase of a liquid-to-gas like phase transition.

\subsection{Pseudo-critical behaviours inside coexistence}

Since the early 80 's, size distributions have been fitted with power laws [84, 85], and more sophisticated critical analysis have been performed following theoretical concepts coming from percolation theory [85]. More recently, an astonishingly 
good scaling behaviour has been observed in the EOS data [72] and tentatively associated to the critical point of the nuclear liquid-gas phase transition expected in nuclear matter [86] in the framework of the Fisher droplet model [84].

Surprisingly enough, a comparable quality of scaling and a consistent set of critical exponents has also been extracted from the same MULTICS-Miniball data [47] for which the heat capacity measurement (see Sect. 2, Chap. 2) points to a first order phase transition.

The debate on the order of the transition has been further animated by a very recent analysis of EOS and Isis data [94] which shows a high quality scaling of the fragment size distribution over a wide range of charges and deposited energies with an ansatz for the scaling function taken from the Fisher droplet model [93]. The critical temperature extracted in these papers from the Fisher analysis is identified as the temperature of the thermodynamical critical point and the whole coexistence line of the nuclear phase diagram is reconstructed under the hypothesis that the Fisher model gives a good description of the multifragmentation phenomenon [94].

However critical exponents and scale invariance are compatible with many different physical phenomena and not necessarily linked to a thermodynamic second order phase transition [87]. In particular the observed signals of critical behaviours can be compatible with a first order phase transition, since in different statistical models size distributions that mimic a scale invariant behaviour are observed inside the coexistence zone of very small systems [11,51,88-91].

Figure 55 shows that the canonical isochore lattice gas model verifies a general property of scale invariance of the cluster size distribution [91] at all densities, even very far from the thermodynamic critical point. In the Fisher droplet model [93] the vapor coexisting with a liquid in the mixed phase of a liquid-gas phase transition is schematized as an ideal gas of clusters. A scaling ansatz of the cluster size distributions around the critical point $\left(T_{c}, \rho_{c}\right)$ is supposed $[93,94]$

$$
n(A, T)=q A^{-\tau} \exp \left(\frac{A \Delta \mu(T)-c_{0}(T) \epsilon A^{\sigma}}{T}\right) .
$$

Here $n(A, \epsilon)=N(A, \epsilon) / A_{0}$ is the cluster distribution normalized to the size of the system, $\tau, \sigma$ are universal critical exponents, $\epsilon=\left(T_{c}-T\right) / T_{c}$ measures the distance from the critical point, $q$ is a normalization constant, $\Delta \mu$ represents the difference in chemical potential between the two phases, and $c_{0}$ is the surface energy coefficient. As it can be seen from Figure 55 [91], a very good scaling is observed for all subcritical as well as supercritical densities. In all cases the values of the critical exponents are comparable, but the extracted critical temperature is a monotonically increasing function of the density. The critical temperatures obtained for each density are represented by the black symbols in the lower part of Figure 55. In this figure the full line gives the coexistence line of the model calculated from the derivatives of the canonical partition sum. Indeed we have discussed in Section 4.2 (Chap. 1) that the first order phase transition in the isochore canonical ensemble corresponds to a backbending of the chemical 

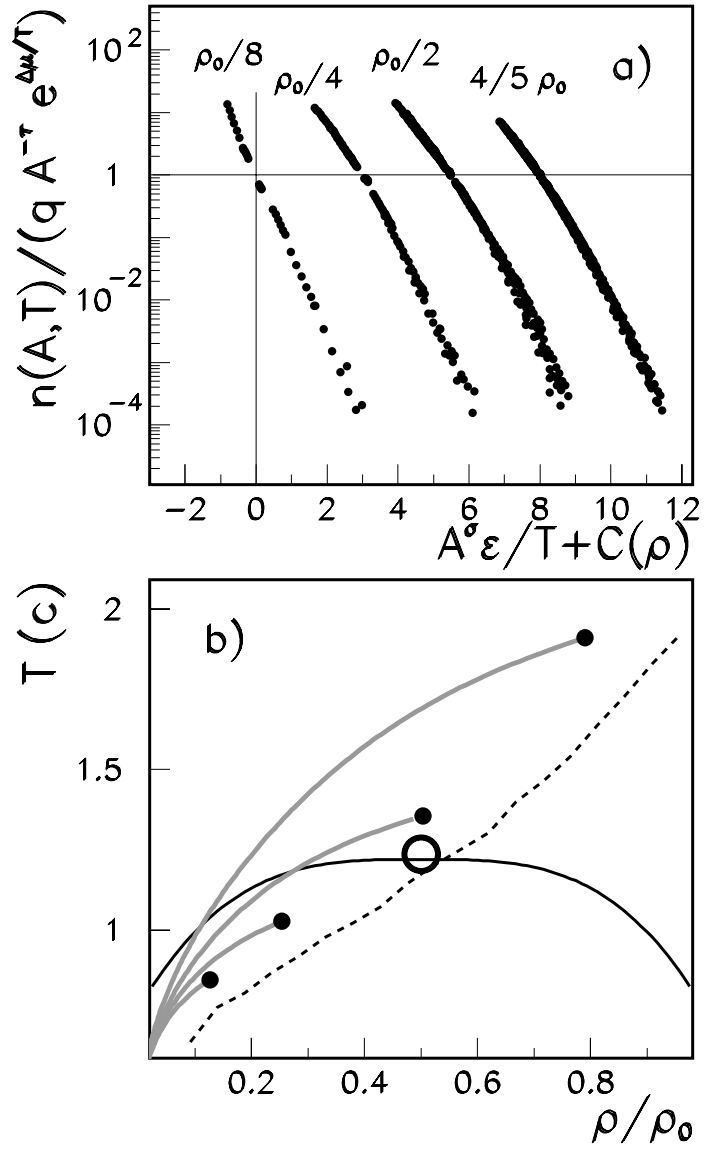

Figure 55. Upper part: fisher scaling of the cluster size distribution from equation (78) in a $8 \times 8 \times 8$ cubic lattice at different densities for temperatures $0.36<T / \epsilon<3.6$ and cluster sizes $4<A<30$. To visualize all the results on the same picture a constant horizontal shift $C(\rho)$ is given to each scaled distribution. Lower part: thermodynamic coexistence line (full line) and region of critical partitions defined from the ansatz (79) (dashed lines). Grey lines: coexistence line reconstructed from fragment partitions with the Fisher model equation of state. Black dots: critical points defined from the Fisher ansatz equation (78) at the different densities represented in the upper part.

potential $\mu=f+\rho \partial_{\rho} f$ where $f$ is the free energy per particle (which can be iteratively evaluated, see Sect. 3.3, Chap. 1). The coexistence line in Figure 55 corresponds to the equality of the chemical potentials $\Delta \mu=0$ on the liquid and gas branch defined by a Maxwell construction $[8,11]$. The locus of criticality lies approximately over a line which passes close to the thermodynamical critical point (open dot) but extends further at supercritical (Kertesz line [31]) as well as subcritical densities inside the coexistence region.

A qualitatively similar behavior (dashed line in Fig. 55) $[8,11]$ is also observed with the scaling ansatz obeyed by three dimensional percolation data

$$
n(A, \epsilon)=A^{-\tau} f\left(\epsilon A^{\sigma}\right) .
$$

At the critical density $\rho_{c}=\rho_{0} / 2$ the scaling temperature results very close to the thermodynamic critical temperature, however a good quality scaling (see Fig. 57 below) is observed also at subcritical $[8,11]$ as well as supercritical [31] densities around a line of points $T_{c}(\rho)$ (dashed line in Fig. 55). 
This result implies that, in the framework of the lattice gas model, the observation of scaling does not allow to localize the critical point and is compatible also with fragment formation at low density inside the coexistence region.

In the work of the Berkely group [94], the whole vapour side of the coexistence line $\rho_{C L}(T)$ is estimated from the results of the Fisher scaling directly from the fragment yields as

$$
\rho_{C L}=\sum_{A} n(A, T) A \exp (-A \Delta \mu),
$$

where the sum extends over all fragments but the biggest. The application of equation (80) to Lattice gas data gives the grey lines in Figure 55 for the four different densities shown in the upper part of the same figure. The end point of the lines giving by construction the total density of the system and the critical temperature extracted by the Fisher fit, these lines are obviously meaningless if the system is fragmenting at a density different from the critical density $\rho_{c}=\rho_{0} / 2$. However even at $\rho=\rho_{c}$, when the thermodynamical critical point is included in the data set, the reconstruction of the coexistence line is very poor. In particular the curvature of this line at the reconstructed critical temperature corresponds to an exponent $\beta=0.84$ which strongly deviates from the expected $\beta=0.31$ exponent given by the thermodynamical coexistence line (full line in Fig. 55) which would be consistent with the liquid gas universality class $(\beta=0.33)$.

This means that, despite the magnificent scaling shown by Figure 55, the physics of the Fisher droplet model does not correspond to the lattice gas. This may look surprising since the main hypotheses of the Fisher model are shared by the lattice: clusters are essentially defined by a volume and a surface contribution; they exhibit a critical behavior at the thermodynamical critical point; the statistical weight of a given configuration is given by a Boltzmann factor.

The two models however strongly differ on one point: if in the Fisher picture fragments constitute an ideal vapor of non interacting composite particles (the individual production probabilities are factorized), in all microscopic models as the lattice gas, interactions among fragments are naturally taken into account through the volume they exclude, and through the surface coupling between neighboring fragments. One may therefore wonder if these interactions, which seem to affect in a non crucial way the inclusive yields, may induce important differences in the thermodynamics. The importance of these effects can be studied by testing the deviation of the Lattice equation of state from the ideal gas hypothesis of the Fisher model. If fragments can be modelized as an ideal classical gas, in a constant volume transformation the pressure can be deduced directly from the fragments yields [94]

$$
\frac{p}{p_{c}}=\frac{\sum_{A} n(A, T)}{\sum_{A} n\left(A, T_{c}\right)} \frac{T}{T_{c}},
$$

where the sum extends over the vapor phase (all fragments but the biggest) and $T_{c}=T_{c}(\rho)$ is the temperature obtained from the Fisher fit for each density (black dots in Fig. 55). This pressure is shown by the open dots in Figure 56 at two different densities and can be compared to the exact pressure of the model 


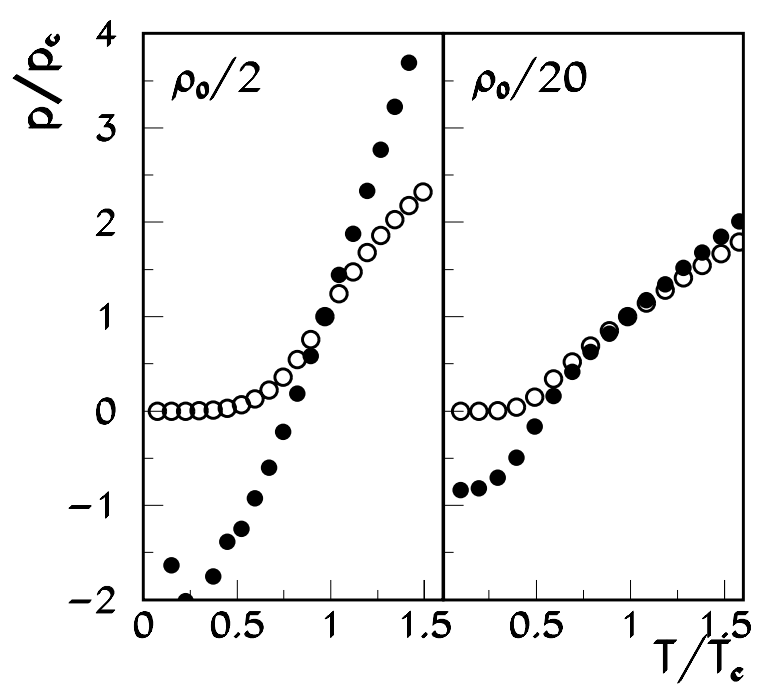

Figure 56. Normalized pressure versus temperature at two different densities from the exact canonical partition sum (full dots) and from the ideal gas approximation equation (81) (open dots).

from reference [11] $p=\rho^{2} \partial_{\rho} f$ (black dots). Not surprisingly, the gas of clusters behaves as an ideal gas only at low density and high temperature. The attractive interaction among fragment surfaces causes the pressure to become negative at low temperatures (the system is bound) while an extra pressure comes from the excluded volume interaction at high density.

To summarize, the quality of scaling does not necessarily imply that the model underlying the scaling ansatz has any pertinence to the data analyzed. Indeed in the case of the lattice gas, the thermodynamics of the lattice is not compatible with the Fisher model, yet a very good quality scaling is observed at all densities.

Concerning experimental data, a comparable quality scaling with the two different ansatz equations $(79,78)$ is also observed in the MULTICS-Miniball data [61]. The invariance of the quality of the scaling with respect to the assumed shape of the scaling function means that scaling "per se" does not demonstrate the existence of a phase transition, and even less defines its order or allows to situate the system on the phase diagram; as a consequence it is not possible to extract the phase diagram from the parameters of the fit in a model independent way. To progress on this point, it is essential to get direct experimental information on thermodynamic quantities $[47,95,96]$ : as we have discussed at length in Section 2 (Chap. 2), these studies rather indicate that multifragmentation can be associated to a first order phase transition.

The evolution of the scaling function and the critical quantities with the size of the system is shown in Figures 57 and 58. At variance with critical behaviours at supercritical density [87], the scalings observed inside the coexistence zone 

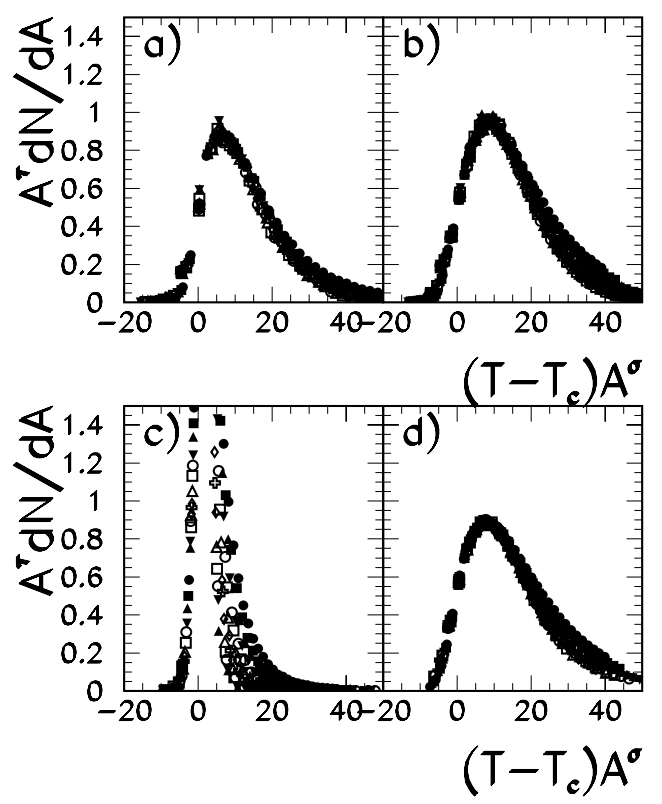

Figure 57. Scaling function at $\rho=\rho_{0} / 4$ (left) and $\rho=\rho_{c}=\rho_{0} / 2$ (right) for clusters of size ranging from $4<A<30$ and temperature $2<T<20$ obtained from equation (79) [8]. The upper (lower) part corresponds to a lattice size $L=8$ $(L=50)$. The coupling $\epsilon$ is fixed to $5.5 \mathrm{MeV}$.
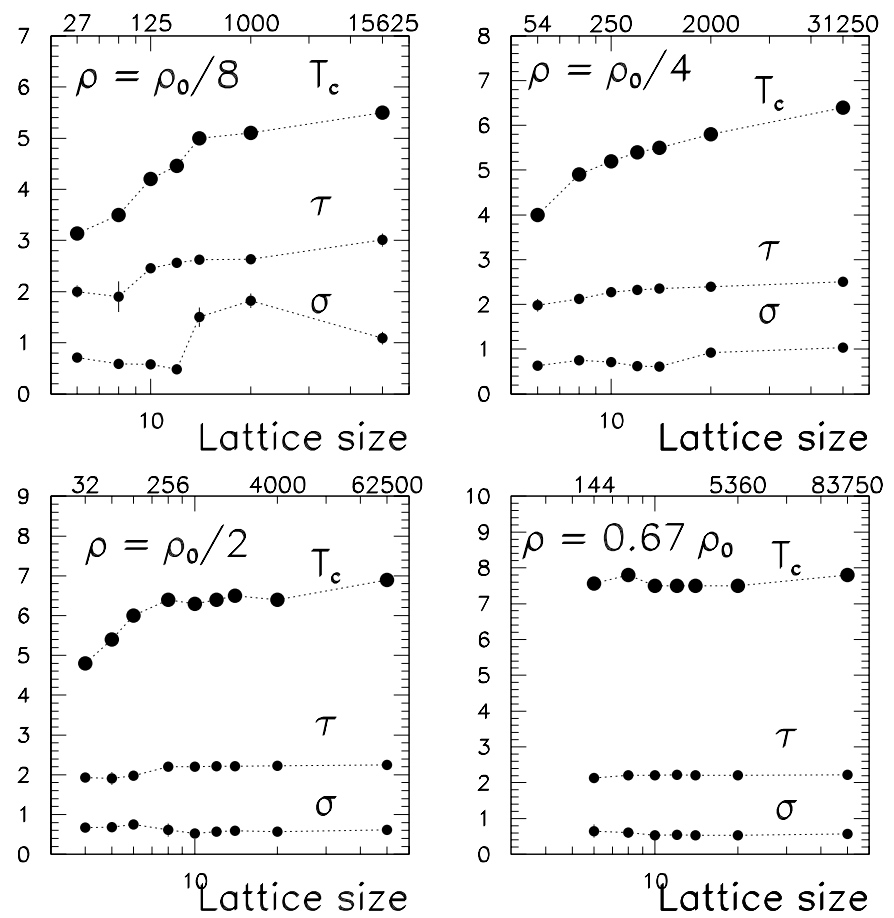

Figure 58. Critical parameters as a function of the linear size of the lattice (lower scale) or of the mass of the system (upper scale) at different densities. The coupling $\epsilon$ is fixed to $5.5 \mathrm{MeV}$. 
do not survive at the thermodynamic limit and are only artifacts of the finite size constraints $[8,11,88,89]$. Indeed we have discussed in Sections 7 (Chap. 1) and 2 (Chap. 2) that the configurational energy fluctuations are maximum in the middle of the coexistence region: such fluctuations correlate points at a distance comparable to the linear size of the system and can therefore have an effect similar to a diverging correlation length in an infinite system. Since these fluctuations are connected to the latent heat of the transition which is a finite quantity, when the system size increases the apparent scale invariance is broken, scaling is violated and the exponents deviate from their universality class. 


\section{Outlooks}

An enormous progress has been done in the analysis of multifragmentation experiments since the first observations of approximate power laws in the inclusive BEVALAC data [84] in the early '80s. Thanks to the last generation sophisticated $4 \pi$ detectors like INDRA or MULTICS, an exclusive analysis of complete events selected in centrality can be performed. The evaluation of collective variables on an event-by-event basis gives access to the high order moments of the observables distribution and allows for the first time to quantitatively address the equations of state of excited nuclear matter.

However it is important to stress that the quantitative study of nuclear thermodynamics is still in its infancy. Since every experimental apparatus has its own bias and deficiencies, the negative heat capacity signals have to be confirmed with experimental measurements with other detectors and only a systematic study as a function of the source mass can asses the isospin effects on the equation of state and the influence of Coulomb.

This is a difficult task, since the problem of data selection is far from being trivial. For a large set of kinematic situations it appears very hard to isolate the preequilibrium particles [97] and/or the collective longitudinal components originated from the neck breaking in the transient bimolecular phase of the heavy ion collision [74]. Since these collective effects result in an overall decrease of fluctuations (see Sect. 1.3, Chap. 1) the negative heat capacity signal can be suppressed in a way which is highly dependent on the entrance channel of the collision and therefore difficult to correct. Indeed the analysis of the quasi-projectile in the INDRA Ta + Au at $32 \mathrm{~A} \mathrm{Mev}$ data, very similar to the MULTICS-Miniball $\mathrm{Au}+\mathrm{Au}$ at $35 \mathrm{~A} \mathrm{MeV}$ system analyzed in Section 2 (Chap. 2), shows a neck emission or dynamical fission component which is not present in the MULTICS data and which leads to partial energy fluctuations lower than the ones of Figure 43 [75]. If this discrepancy can be understood from the theoretical point of view (see Sect. 1.3, Chap. 2), however it shows that the different detectors can produce a bias on the statistical sample which is difficult to control. In this respect it is interesting to know that the ISIS $\pi+\mathrm{Au}$ data at $600 \mathrm{~A} \mathrm{MeV}$ for which the dynamics of the entrance channel is irrelevant, seem to show a very clean abnormal fluctuation signal [63] closely comparable with the MULTICS one. In this set of data however the evaporation residue is systematically undetected and light charged particle 
spectra show important high energy preequilibrium tails, and careful simulations have to be performed to clearly assess the effect of these limitations.

Apart from the abnormal fluctuation observable, in the last years we assist to an impressive accumulation of different signals of phase transitions in excited nuclei $[38,94,96,98]$. Since the bias of the different signals are in general very different (see Sect. 1.3, Chap. 2), only the correlation among the different observables can bring a definitive convincing bunch of evidences of the phase transition.

Independent of the problems of data analysis, the important conceptual problem of equilibrium is also still open. If we could dispose of an exact dynamical theory of nuclear collisions depending on just a few free parameters adjustable from experimental data, the nuclear equation of state could be accessed by a theoretical study of the equilibrium properties of this dynamical theory [99] with the help of the ergodic theorem. Because of the enormous complexity of the quantum many body problem this aim is most probably impossible to reach, even if in the recent years many body quantum transport theories have greatly progressed.

The information theory approach that we have developed all along this work (see in particular Sect. 1, Chap. 2) is extremely powerful, however it is important to stress that, in spite of its formal elegance and theoretical consistency, the predictive power of this theory can never be guaranteed. If we can measure a mean value and nothing else, this does not necessarily imply that the distribution of the corresponding observable is such as to maximize the underlying entropy, even if this is certainly the only reasonable working hypothesis that we have at hand. In other words, the fact that we dispose of a limited information on a data sample does not ensure that the objective physical information is effectively limited. This is why the degree of realization of a given equilibrium (i.e. the minimization of information under a given finite set of dynamical constraints) has always to be checked by comparing the data set with other data, and with statistical models that contain the same constraints.

It is important to realize that equilibrium, being by definition the random realization of all the possible microstates of the system, is never exactly attained. For a statistical description to be pertinent, a statistical model does not need to reproduce the whole set of observables that can be defined on the data sample, but only to be adequate for the specific observables we wish to study. For instance if we want to perform a fluctuation analysis in view of the measurement of the heat capacity, the statistical model must reproduce the data at the level of the second moments of collective observables, while deviations on single particle observables or higher order moments can be safely accepted.

Even this is not easy to check. Indeed statistical models that can be exactly solved as Ising-like lattice models, because of their classical nature cannot be reasonably compared to nuclear data in a quantitative way, while macroscopic fragmentation models with an energetics and state density fitted from nuclear data $[48,79]$ use strong simplifications and approximations in the sampling procedure and the absence of bias of the statistical sampling is not clearly demonstrated.

This discussion on equilibrium may be academic as long as the nuclear interaction is concerned. Indeed model calculations employing short range forces 
systematically show chaotic features and high sensitivities to the initial conditions, which suggests that the ensemble of collisional events of identically prepared system (i.e. corresponding to the same impact parameter) can be identified with a Gibbs statistical ensemble. However we cannot a priori exclude that the population of phase space under the dynamical constraints is not uniform: this is even highly probable for the Coulomb part of the energy, since the relaxation time of long range interactions can diverge $[78,100]$. For example if the fragment production mechanism is based on the spinodal decomposition [98], the Coulomb interaction is not expected to influence the fragment partitions at variance with an equilibrium picture, due to the insensitivity of the short unstable wavelengths to the long range interactions. This picture would be consistent with the astonishingly good scaling with the size of the system of multifragmentation partitions $[56,61]$.

It has been proposed [3] that these effects can be accounted for, using an information kernel different from the standard exponential of the Gibbs theory that we have presented in Section 1 (Chap. 1) and used throughout this paper. Indeed the use of the so called non extensive $q$-statistics [3] proposed by Tsallis, produces a distribution function for an observable controlled in average which can be different from the Gibbs distribution, depending on the value of a single parameter $q$, while the main characteristics of the statistical entropy are preserved. Values of $q>1(q<1)$ enhance (suppress) the tails of the distribution while $q=1$ gives back the Gibbs distribution.

It is interesting to note that in the case of a Tsallis equilibrium the topologic definition of phase transitions developed in Section 6 (Chap. 1) can still be applied. An example from the isobar lattice gas model is given in Figure 59. The energy distribution characterized by the two parameters $\beta$ and $q$,

$$
N_{\beta q}(E)=Z_{\beta q}^{-1} W(E)(1-(1-q) E / \beta)^{q / q-1}
$$

is shown at different pressures for $q<1, q>1$ and $q=1$. Deep inside the coexistence (left part of Fig. 59) the bimodality signal persists in the Tsallis ensemble, however close to the critical point the Tsallis distribution can be qualitatively different from the Gibbs one [101]. This is clearly shown by Figure 59. In the $q<1$ case the distribution shows no inversion of concavity in a region where the density of states has an anomaly (left part of Fig. 59), as it can be seen from the fluctuation properties of the system (middle left). Conversely in the case $q>1$ the distribution is still bimodal even at the critical pressure [101] (right part of Fig. 59). In the framework of an extended theory of phase transitions (see Sect. 6, Chap. 1) this bimodality can indeed be interpreted as an actual phase transition in the non extensive ensemble. If, following the theoretical proposition of Section 6 (Chap. 1), we still interpret the logarithm of the probability distribution as an entropy even out of a Gibbs equilibrium, the second derivative of this quantity represents a (non-extensive) heat capacity, shown in the lowest part of Figure 59. Then we can conclude that the phase transition still exists out of equilibrium, the effect of $q$-statistics being summarized by a shift of the critical point. 

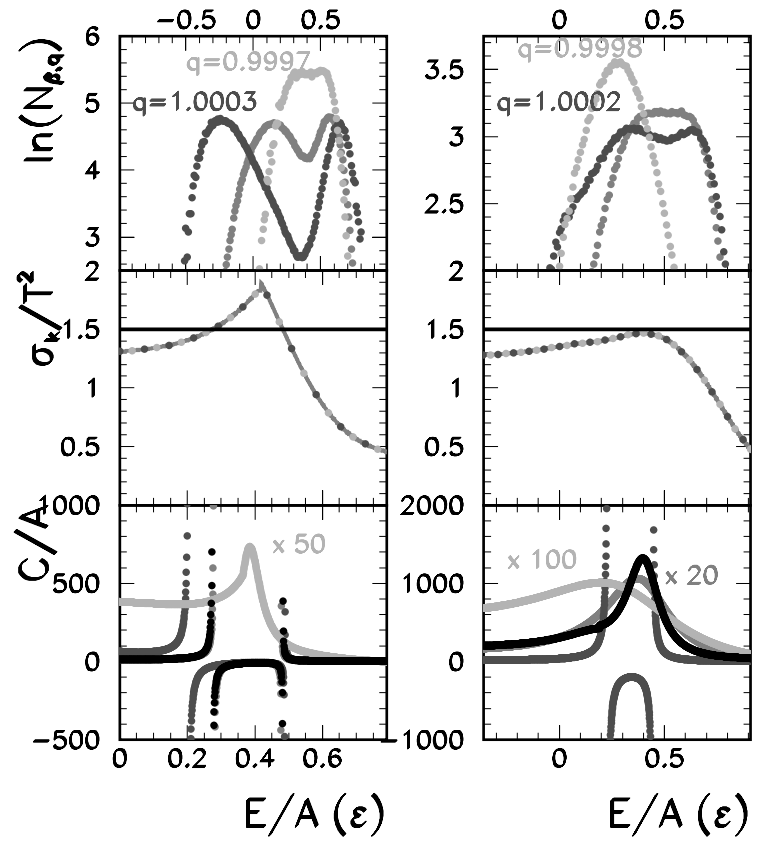

Figure 59. Upper part: energy distribution inside the coexistence region for the canonical (medium grey) and the Tsallis (light grey: $q<1$, dark grey: $q>1$ ) ensemble. Medium part: normalized kinetic energy fluctuations (symbols) and kinetic heat capacity (horizontal line). Lower part: microcanonical heat capacity calculated from fluctuations (black) and from the entropies deduced from the energy distributions (light, medium and dark grey). The external pressure is below $p_{c}$ (left side), and equal to $p_{c}$ (right side).

However it is important to remark that in the $q \neq 1$ case the probability distribution does not allow to extract any information about the density of states, at variance with the discussion in Section 6 (Chap. 1). Conversely microcanonical information can be extracted from the study of partial energy fluctuations (see Sect. 7, Chap. 1), since the important property of the microcanonical ensemble is that it can be accessed from any equilibrium by sorting events in energy bins. The kinetic energy variance of the lattice gas model is compared to the canonical expectation in the medium panels of Figure 59 (black curve). The same abnormal fluctuations are observed in the coexistence zone independent of $q$. The corresponding heat capacity evaluated from equation (66) is displayed in the lower part of Figure 59. This example shows that a systematic correlation between bimodalities and fluctuations could allow to infer non extensif effects in the information kernel. As discussed in Section 1.3 (Chap. 2) above, the same thing is true for less exotic memory effects in the entrance channel. 


\section{References}

[1] E.T. Jaynes, Phys. Rev. 106, 620 (1957); Phys. Rev. 108, 171 (1957).

[2] A. Katz, Principles of statistical mechanics (Freeman, 1967).

[3] C. Tsallis et al., Phys. A 261, 534 (1988); S. Abe, Y. Okamoto, Nonextensive statistical mechanics and its applications, Lecture Notes in Physics (World Scientifique, 2001), Vol. 560.

[4] R. Balian, From microphysics to macrophysics (Springer Verlag, 1982).

[5] Ph. Chomaz, Ann. Phys. Fr. 21, 669 (1996).

[6] L. Onsager, Phys. Rev. 65, 117 (1944).

[7] E. Marinari, Nucl. Phys. B 235, 123 (1984).

[8] Ph. Chomaz, F. Gulminelli, Int. Journ. Mod. Phys. E 8, 527 (1999).

[9] M.E. Fisher, Critical phenomena (Academic, 1971).

[10] L. Van Hove, Physica 15, 951 (1949); C.N. Yang, T.D. Lee, Phys. Rev. 87, 404 (1952); K. Huang, Statistical Mechanics (John Wiley and Sons Inc., 1963), Chap. 15.2 and Appendix C.

[11] Ph. Chomaz, F. Gulminelli, Phys. Rev. Lett. 82, 1402 (1999).

[12] M.S. Challa, J.H. Hetherington, Phys. Rev. Lett. 60, 77 (1988); M.S. Challa, J.H. Hetherington, Phys. Rev. A 38, 6324 (1988).

[13] D.H.E. Gross, Phys. Rep. 279, 119 (1997); Microcanonical thermodynamics: phase transitions in finite systems, Lecture notes in Physics (World Scientific, 2001), Vol. 66.

[14] F. Gulminelli, Ph. Chomaz, Europhys. Lett. 50, 434 (2000); V. Duflot, Ph. Chomaz, F. Gulminelli, Phys. Lett. B 476, 279 (2000).

[15] F. Gulminelli, Ph. Chomaz, Phys. Rev. Lett. 85, 3587 (2000). 
[16] T.L. Beck, R.S. Berry, Journ. Chem. Phys. 88, 3910 (1988); M. Bixon, J. Jortner, Journ. Chem. Phys. 91, 1631 (1989); P. Labastie, R.L. Whetten, Phys. Rev. Lett. 65, 1567 (1990).

[17] D.J. Wales, R.S. Berry, Phys. Rev. Lett. 73, 2875 (1994); R.E. Kunz, R.S. Berry, J. Chem. Phys. 103, 1904 (1995); D.J. Wales et al., Adv. Chem. Phys. 115, 1 (2000); O. Muelken, H. Stamerjohanns, P. Borrmann, Phys. Rev. E 64, 047105 (2001).

[18] L. Moretto et al., Phys. Rep. 287, 249 (1997); K. Binder, Physica A 319, 99 (2003).

[19] F. Gulminelli et al., Phys. Rev. E 68, 026120 (2003).

[20] V. Antonov, Vest. Leningrad Univ. 7, 135 (1962).

[21] D. Lynden-Bell, Monthly Not. Roy. Astr. Soc. 138, 495 (1968).

[22] W. Thirring, Z. Phys. 235, 339 (1970).

[23] T. Padmanabhan, Phys. Rep. 188, 285 (1990).

[24] P.H. Chavanis, Phys. Rev. E 65, 056123 (2002).

[25] C.N. Yang, T.D. Lee, Phys. Rev. 87, 410 (1952).

[26] K. Binder, D.P. Landau, Phys. Rev. B 30, 1477 (1984).

[27] P. Labastie, R.L. Whetten, Phys. Rev. Lett. 65, 1567 (1990).

[28] Ph. Chomaz, F. Gulminelli, V. Duflot, Phys. Rev. E 64, 046114 (2001).

[29] R.M. Lynden-Bell, Mol. Phys. 86, 1353 (1995).

[30] A. Coniglio, W. Klein, J. Phys. A 13, 2775 (1980).

[31] X. Campi, H. Krivine, A. Puente, Physica A 262, 328 (1999).

[32] T. Poston, I. Stewart, Catastrophe Theory and its applications (Pitman, 1978).

[33] Ph. Chomaz, F. Gulminelli, Physica A 330, 451 (2003).

[34] S. Grossmann, W. Rosenhauer, Z. Phys. 207, 138 (1967).

[35] K.C. Lee, Phys. Rev. E 53, 6558 (1996); J. Lee, K.C. Lee, Phys. Rev. E 62, 4558 (2000).

[36] M.S.S. Challa et al., Phys. Rev. B 34, 1841 (1986).

[37] P. Borrmann et al., Phys. Rev. Lett. 84, 3511 (2000); H. Stamerjohanns et al., Phys. Rev. Lett. 88, 053401 (2002).

[38] O. Lopez et al., Nucl. Phys. A 685, 246 (2001). 
[39] J. Barré, D. Mukamel, S. Ruffo, Phys. Rev. Lett. 87, 030601-1 (2001); F. Leyvraz, S. Ruffo, J. Phys. A 35, 285 (2002).

[40] L. Casetti, M. Pettini, E.G.D. Cohen, Phys. Rep. 337, 237 (2000).

[41] F. Gulminelli, Ph. Chomaz, Phys. Rev. E 66, 046108 (2002).

[42] J.L. Lebowitz, J.K. Percus, L. Verlet, Phys. Rev. 153, 250 (1967).

[43] J.A. Reyes-Nava, I.L. Garzon, K. Michaelian, Phys. Rev. B 67, 165401 (2003).

[44] E.M. Pearson, T. Halicioglu, W.A. Tiller, Phys. Rev. A 32, 3030 (1985); Al.H. Raduta, Ad.R. Raduta, Nucl. Phys. A 647, 12 (1999); J. Jellinek, A. Goldberg, Journ. Chem. Phys. 113, 2570 (2000).

[45] P. Chomaz, F. Gulminelli, Nucl. Phys. A 647, 153 (1999).

[46] X. Campi, H. Krivine, Nucl. Phys. A 620, (1997); J.M. Carmona et al., Phys. Rev. C 61, 037304 (2000).

[47] M. D'Agostino et al., Nucl. Phys. A 650, 329 (1999); Phys. Lett. B 473, 219 (2000); Nucl. Phys. A 699, 795 (2002).

[48] J.P. Bondorf, A.S. Botvina, A.S. Iljinov, I.N. Mishustin, K. Sneppen, Phys. Rep. 257, 133 (1995).

[49] R.J. Charity et al., Nucl. Phys. A 483, 371 (1988).

[50] J.P. Bondorf, A.S. Botvina, I.N. Mishustin, Phys. Rev. C 58, R27 (1998); J.B. Elliott, A.S. Hirsch, Phys. Rev. C 61, 054605 (2000).

[51] K.A. Bugaev et al., Phys. Rev. C 62, 044320 (2000); Phys. Lett. B 498, 144 (2001).

[52] R.C. Tolman, Principles of Statistical Mechanics (Oxford University press, London, 1962).

[53] M. Pleimling, A. Hueller, J. Stat. Phys. 104, 971 (2001).

[54] F. Rami et al., Phys. Rev. Lett. 84, 1120 (2000).

[55] W. Reisdorf, Progr. Theor. Phys. Suppl. 140, 111 (2000).

[56] M.F. Rivet et al., Phys. Lett. B 430, 217 (1998); J.D. Frankland et al., Nucl. Phys. A 689, 905 (2001).

[57] D. Jou et al., Thermodynamics of fluids under flow (Springer, 2001).

[58] W. Norenberg, G. Papp, P. Rozmej, Eur. Phys. J. A 9, 327 (2000).

[59] X. Campi, H. Krivine, N. Sator, Nucl. Phys. A 681, 458 (2001). 
[60] C.B. Das, S. Das Gupta, Phys. Rev. C 64, 041601 (2001).

[61] M. D'Agostino et al., Nucl. Phys. A 724, 455 (2003).

[62] N. Marie et al., Phys. Lett. B 391, 15 (1997).

[63] ISIS collaboration, private communication.

[64] A.J. Cole, Statistical models for nuclear decay (Institute of Physics publishing, Bristol and Philadelphia, 2002), Chap. 5.

[65] M. D'Agostino et al., contribution to the XXXVIII Winter Meeting on Nucl. Phys., Bormio (Italy), edited by I. Iori, A. Moroni (2000), p. 386.

[66] N. Marie, A. Chbihi, J.B. Natowitz, A. Le Fèvre et al., Phys. Rev. C 58, 256 (1998); A. Chbihi et al., contribution to the XXXVIII Winter Meeting on Nucl. Phys., Bormio (Italy), edited by I. Iori (2000), p. 404.

[67] T. Glasmacher et al., Phys. Rev. C 51, 3489 (1995).

[68] S. Das Gupta, A.Z. Mekjian, B. Tsang, Adv. Nucl. Phys. 26, 89 (2002).

[69] P.M. Milazzo et al., Phys. Rev. C 58, 953 (1998).

[70] M. D'Agostino et al., Nucl. Phys. A 724, 455 (2003); M. D'Agostino et al., Nucl. Phys. A 734, 512 (2004).

[71] P. Desesquelles et al., Nucl. Phys. A 633, 547 (1998); P.M. Milazzo et al., Phys. Rev. C 66, 021601(R) (2002).

[72] J.B. Elliott et al., Phys. Lett. B 418, 34 (1998); R.P. Scharenberg et al., Phys. Rev. C 64, 054602 (2001).

[73] E. Vient et al., Nucl. Phys. A 700, 555 (2002).

[74] F. Bocage et al., Nucl. Phys. A 676, 391 (2000); J. Colin et al., Phys. Rev. C 67, 064603 (2003).

[75] INDRA collaboration, private communication.

[76] P. Bonche, S. Levit, D. Vautherin, Nucl. Phys. A 436, 265 (1985).

[77] S.J. Lee, A.Z. Mekjian, Phys. Rev. C 63, 044605 (2001).

[78] V. Latora, A. Rapisarda, C. Tsallis, Phys. Rev. E 64, 056134 (2001).

[79] Al.H. Raduta, Ad.R. Raduta, Phys. Rev. C 55, 1344 (1997); Al.H. Raduta, Ad.R. Raduta, Phys. Rev. C 56, 2059 (1997); Al.H. Raduta, Ad.R. Raduta, Phys. Rev. C 61, 034611 (2000).

[80] S. Siem et al., Phys. Rev. C 65, 044318 (2002). 
[81] M. Belkacem et al., Phys. Rev. C 52, 271 (1995); J. Carmona et al., Eur. Phys. Journ. A 11, 87 (2001); Al.H. Raduta, Ad.R. Raduta, Nucl. Phys. A 703, 876 (2002).

[82] R. Botet, M. Ploszajczak, Phys. Rev. E 62, 1825 (2000).

[83] M. Pichon, B. Tamain et al., contribution to the XLI Winter Meeting on Nucl. Phys., Bormio (Italy), edited by I. Iori, A. Moroni (2003); M. Pichon et al., Nucl. Phys. A 749, 93 (2005).

[84] J.E. Finn et al., Phys. Rev. Lett. 49, 1321 (1982); A.S. Hirsch et al., Nucl. Phys. A 418, 267 (1984); M. Mahi et al., Phys. Rev. Lett. 60, 1936 (1988); A.D. Panagiotou, M.W. Curtin, H. Toki, D.K. Scott, P.J. Siemens, Phys. Rev. Lett. 52, 496 (1984).

[85] X. Campi, J. Desbois, E. Lipparini, Phys. Lett. B 138, 353 (1984).

[86] G.F. Bertsch, P.J. Siemens, Phys. Lett. B 126, 9 (1983).

[87] X. Campi, Phys. Lett. B 208, 351 (1988); X. Campi, H. Krivine, Z. Phys. A 344, 81 (1992); X. Campi, H. Krivine, N. Sator, Nucl. Phys. A 681, 458 (2001).

[88] C.B. Das, S. Das Gupta, A. Majumder, Phys. Rev. C 65, 34608 (2002); C.B. Das et al., Phys. Rev. C 66, 044602 (2002).

[89] J.M. Carmona, J. Richert, P. Wagner, Phys. Lett. B 531, 71 (2002).

[90] A.H. Raduta et al., Phys. Rev. C 65, 034606 (2002).

[91] F. Gulminelli et al., Phys. Rev. C, 51601 (2002).

[92] D. Stauffer, A. Aharony, Introduction to percolation theory (Taylor and Francis, London, 1992).

[93] M.E. Fisher, Physics 3, 255 (1967).

[94] J.B. Elliott et al., Phys. Rev. Lett. 88, 042701 (2002); J.B. Elliott et al., Phys. Rev. C 67, 024609 (2003).

[95] J. Pochodzalla et al., Phys. Rev. Lett. 75, 1040 (1995).

[96] J.B. Natowitz et al., Phys. Rev. C 65, 34618 (2002).

[97] B. Zwieglinski et al., Nucl. Phys. A 681, 275 (2001).

[98] M. Colonna et al., Phys. Rev. Lett. 88, 122701 (2002); B. Borderie et al., Phys. Rev. Lett. 86, 3252 (2001).

[99] H. Feldmeier, Nucl. Phys. A 681, 398 (2001).

[100] T. Dauxois et al., Dynamics and Thermodynamics of systems with long range interactions, Lecture Notes in Physics (Springer, 2002), Vol. 602.

[101] K.K. Gudima et al., Phys. Rev. Lett. 85, 4691 (2000). 